\title{
BEAM ASYMMETRY FOR PHOTOPRODUCED $\omega$ MESONS OFF BOUND PROTONS IN DEUTERONS
}

\author{
by \\ Olga María Cortés Becerra
}

\author{
A dissertation \\ submitted in a partial fulfilment \\ of the requirenments for the degree of \\ Doctor of Philosophy in the Department of Physics \\ Idaho State University
}

Spring 2018 


\section{Committee Approval}

To the Graduate Faculty:

The members of the committee appointed to examine the dissertation of Olga María Cortés Becerra find it satisfactory and recommend that it be accepted.

Dr. Philip L. Cole, Major Advisor

Dr. Daniel S. Dale, Committee Member

Dr. David Delehanty, Graduate Faculty Representative

Dr. Tony Forest, Committee Member

Dr. Dustin McNulty, Committee Member 


\section{Acknowledgments}

I would like to acknowledge the contributions of the people and institutions that helped me not only in the completion of this work, but also guided me through the entire life experience that was this doctorate. First, I would like to thank the members of my committee for their support and advice at various stages of this research work. I am particularly grateful to my adviser, Professor Philip Cole, for his guidance and for the independence that he gave me throughout the course of my graduate program. Even with his very busy schedule, Dr. Cole had always his door open for me and my physics questions. I would like to thank him also for giving me the opportunity to move to Newport News to have a first hand experience at JLab. Also I will be always indebted to him for his total support during my maternity leaves.

I would like to thank my colleagues of the g13 group, in particular Pawel Nadel-Turonski and Yordanka Ilieva, for all the discussions and suggestions. To Paul Mattione, my dear friend, for giving me all the possible advice with the g13 software, but also for the walks, the ping pong games and the great talks. I would like to thank Nicholas Zachariou and Daria Sokhan for their advice, physics and nonphysics related. To Chuck Taylor that gave me a hand at the beginning of my thesis; with his selfless help I was able to start analyzing data right away. Thanks also to Colin, Tongtong and Nick C.

During my first years in the PhD program, the discussions and company of my two friends, Danny Martinez and Carlos "el corsario" Bula, helped me adapting to the graduate school. I am very thankful with them. I would like to thank my dear friend Oleksiy Kosinov, for all the time that we shared walking, swimming

and running in Idaho. Also for the days trying to figure out Geant4 and Root, and 
the discussions about physics. I am greatful to Bindu, Bibek, Shraddha, George K, Maya, George T, Kevin, Valeriia, and Misha for making Idaho a warmer place. During my stay at JLab I had the great opportunity to collaborate with Mac Mestayer. I loved working with Mac, and I am thankful for the good time and hard work that we did together. I am also thankful to Veronique Ziegler and Gagik Gavalian for all the patience that they had when I had thousands of questions about JAVA. I appreciate the great advice I got from Latifa Elouadrhiri and Patrizia Rossi about my work and my carreer. To my friends Carlitos, Juan, Liliana, Cesar, Nobuo, David, Raul, Dave, Christian, Melissa, Mark, Will and the soccer people for making my life in Virginia very enjoyable.

Lastly, I thank my family. Mamma, muchas gracias por todo el apoyo incondicional que siempre me has dado. Gracias por darme tu mano y guiarme incluso en los momentos de mayor oscuridad. Gracias totales a mi padre que no vivio tan largo para ver esto, pero que este trabajo es tambien fruto de todo lo que sembro. Gracias a mis queridas hermanas Elizabeth, Beatriz y Diana por ser un trio de mujeres a quienes admiro profundamente y quienes me mueven a querer mejorar. I am thankful to my husband, my daughter Leila and my son Ibai, for the patience for all the nights of absence trying to write this thesis, and for the days that I was not myself. Carlos, thank you for believing in me and for being my rock during all this time. 


\section{Contents}

List of Figures $\quad$ x

List of Tables $\quad$ xviii

Abstract $\quad$ xix

Chapter 1: Hadron Spectrum 1

1.1 Baryon Spectroscopy . . . . . . . . . . . . . . . 1

1.1.1 Photoproduction of Vector Mesons . . . . . . . . . 6

$\begin{array}{lll}\text { Chapter 2: } & \text { Photoproduction of } \omega \text { Mesons } & 8\end{array}$

2.1 Vector Meson Photoproduction .............. . 9

2.2 Previous Measurements for $\omega$ Photoproduction . . . . . . . . . . . 10

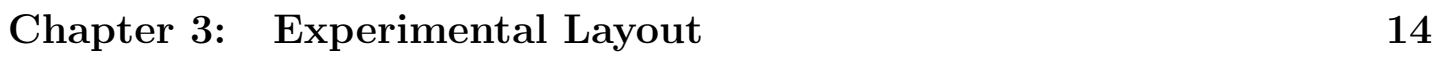

3.1 Electron Beam ...................... 15

3.2 Coherent Bremsstrahlung Facility . . . . . . . . . . . . . . . 18

3.2.1 Coherent Bremsstrahlung . . . . . . . . . . . 18

3.2.2 Diamond Radiator and Goniometer . . . . . . . . . . . . . 21

3.2.3 Active Collimator . . . . . . . . . . . . . . . . 23

3.2.4 Photon Tagger .................. 23

3.3 CLAS Detector . . . . . . . . . . . . . . 25

3.3.1 Torus Magnet .................. 26

3.3.2 Start Counter .................... 28 
3.3.3 Drift Chambers . . . . . . . . . . . . . . . . . . . 30

3.3 .4 Time of Flight . . . . . . . . . . . . . . . . . . . . . . 31

3.3.5 Electromagnetic Calorimeter . . . . . . . . . . . . . . . . 32

3.4 The g13b Experiment . . . . . . . . . . . . . . . . . . . 34

3.4 .1 Target Cell . . . . . . . . . . . . . . . . . . . 34

3.4 .2 Trigger Conditions . . . . . . . . . . . . . . . 35

$3.4 .3 \quad \mathrm{DAQ} \ldots \ldots \ldots \ldots \ldots \ldots \ldots \ldots$

Chapter 4: Data Analysis: Event Reconstruction 37

4.1 Overview . . . . . . . . . . . . . . . . 37

4.2 "Cooking" and Detector Calibration _. . . . . . . . . . 38

4.3 Data Exclusion . . . . . . . . . . . . . . . . . . . 41

4.4 Skimming . . . . . . . . . . . . . . . . . . . . 42

4.4 .1 First SKIM . . . . . . . . . . . . . . . . . 42

$4.4 .2 \quad$ Second SKIM . . . . . . . . . . . . . . . . . . . 42

4.5 Particle Identification . . . . . . . . . . . . . . . . . . . . . 43

4.5 .1 Charged Particles . . . . . . . . . . . . . . . . 43

4.5.2 Neutral Particle Identification . . . . . . . . . . . . 46

4.6 Incoming Photon Identification $\ldots \ldots \ldots \ldots$

4.7 Hadron Time Coincidence . . . . . . . . . . . . . . . . . . . 51

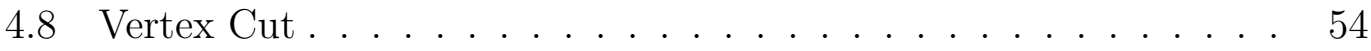

$4.9 \pi^{0}$ Reconstruction . . . . . . . . . . . . . . . . . . 54

4.10 Fiducial Cuts . . . . . . . . . . . . . . . 56

4.11 Energy and Momentum Corrections . . . . . . . . . . . 57

4.11 .1 Energy Loss Corrections . . . . . . . . . . . . . . 57

4.11 .2 Momentum Corrections . . . . . . . . . . . . . . . . . 58 
4.11.3 Incoming Photon Energy Corrections . . . . . . . . . . . . 59

4.12 Missing Momentum Cut . . . . . . . . . . . . . . . . . 61

$4.13 \omega$ Meson Reconstruction . . . . . . . . . . . . . . . . . . . 62

Chapter 5: Data Analysis: Beam Asymmetry 66

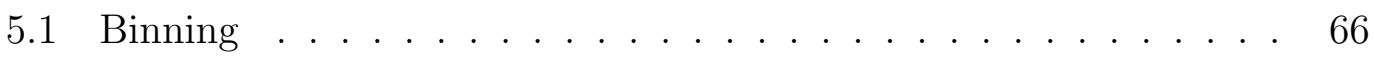

5.2 Beam Asymmetry: the $\phi$ Binned Method . . . . . . . . . . . . . 69

5.2.1 Parameter Optimization ............. 71

5.2.2 Dilution Factor. . . . . . . . . . . . . . . . . . 75

5.3 Statistical Uncertainty . . . . . . . . . . . . . . . . . 78

5.4 Results . . . . . . . . . . . . . . . . . . . . 79

Chapter 6: $\quad$ Systematic Studies $\quad 82$

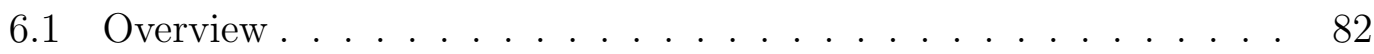

6.2 Goodness of the Fit . . . . . . . . . . . . . . 83

6.3 Systematics of the Parameters . . . . . . . . . . . . . . 84

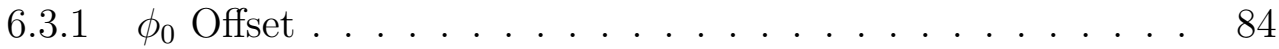

6.3.2 Photon Flux . . . . . . . . . . . . . . 85

6.3.3 Photon Polarization Ratio . . . . . . . . . . . 85

6.4 Event Selection Systematic Uncertainty . . . . . . . . . . . . 86

6.4.1 Neutral Particle Identification Systematics . . . . . . . . . 87

6.4 .2 Incident Photon Identification . . . . . . . . . . . . . 87

6.4.3 Out-of-Time Cut ................ 88

6.4.4 z-Vertex Cut ....................... 89

6.4.5 Missing Momentum . . . . . . . . . . . . . 89

6.5 Systematic due to Dilution Factor and Invariant Mass Cut . . . . 90

6.6 Summary and Conclusion . . . . . . . . . . . . . . . 91 
6.6.1 Systematic Error Budget . . . . . . . . . . . . . . 91

$\begin{array}{lll}\text { Chapter 7: } & \text { Summary and Conclusions } & 93\end{array}$

$\begin{array}{ll}\text { Bibliography } & 95\end{array}$ 


\section{List of Figures}

1.1 Total photo-absorption cross section and exclusive cross sections for different channels as a function of the photon energy. . . . . . 4

1.2 Breit-Wigner masses and widths of $N^{*}$ resonances. Table taken from [4] with permission. . . . . . . . . . . . . . 5

1.3 Main mechanisms in the $\omega$ photoproduction using real photons. From left to right: $t$-channel pomeron exchange, $t$-channel pion exchange, $s$-channel $N^{*}$ resonance. . . . . . . . . . . . 6

2.1 Photon beam asymmetry $\Sigma$ as a function of $\cos \theta_{\mathrm{CM}}$ for the reaction $\vec{\gamma} p \rightarrow \omega p$ for photon energy $E_{\gamma} \in[1.152,1.876] \mathrm{GeV}$ as reported in [14]. The black squares are the result of CLAS 2017 study, it shows only statistical error and not a $6 \%$ systematic uncertainty for each energy bin. The open red squares are the results from the CBELSA/TAPS [15], the blue open open circles and the red solid circles from the GRAAL 2006 [16] and GRAAL 2015 [17]

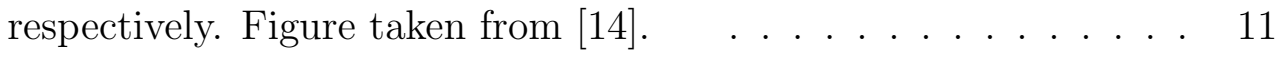


2.2 Beam asymmetry $\Sigma$ as a function of $\theta_{C M}$. The full circles represent the free proton channel, the full triangles the quasifree proton in a deuteron target for $E_{\gamma} \in[1.18,1.48] \mathrm{GeV}$. Only statistical uncertainties are shown in the figure and reported in the paper. The lines represent the Zhao model described in [18-20]. Figure taken from $[17] . \ldots \ldots \ldots \ldots \ldots$

3.1 Aerial view of the CEBAF at the Thomas Jefferson National Accelerator Facility (JLab). Picture taken from [21]. . . . . . . . .

3.2 Experimental Layout of the CEBAF accelerator before the $12 \mathrm{GeV}$

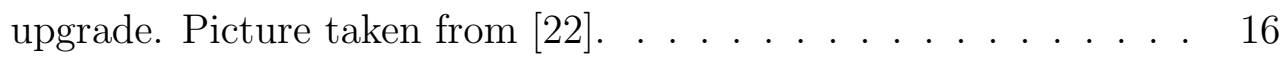

3.3 Photo of a Niobium Cavity. . . . . . . . . . . . . . . . 17

3.4 Charge distribution in the cavity. . . . . . . . . . . . . . 17

3.5 Picture of the magnets in the arcs at Jefferson Lab. . . . . . . . 18

3.6 Energy spectrum of linearly polarized photons, showing the coherent enhancement over the incoherent background. Data were taken during the g13b experiment, for a coherent edge of $2.1 \mathrm{GeV}$.

3.7 Typical enhancement plot. Taken from [25]. Copyright 2018, with permission from Elsevier. . . . . . . . . . . . . . . . . 21

3.8 Goniometer . . . . . . . . . . . . . . . . . 22

3.9 Photon tagger. ....................... 25

3.10 Experimental Layout of the CLAS detector before the $12 \mathrm{GeV}$

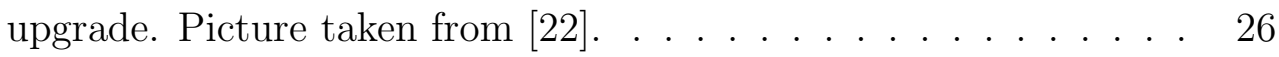


3.11 a. Magnetic Field contour plot in the middle plane between coils. b. Magnetic field perpendicular to the beamline and centered in the target. The six coils can be seen in the intersection with the plane. Image taken from [22] with permission. . . . . . . . . . 27

3.12 Schematic view of the torus cryostat. Image taken from [22] with permission. ....................... 28

3.13 Schematic 3D view of the start counter. The purple volume represents the target cell. Image taken from [29] with Elsevier permission. 29

3.14 Schematic cross section of the start counter subsystem. Image taken from [29] with Elsevier permission. . . . . . . . . . . 29

3.15 Schematic 2D view of the CLAS detector cut at the beamline high. It shows a representation of two charged particles being detected. The dotted lines represent the projection on this plane of the torus coils position. Enlargement in the box exemplifies the hit pattern in the two superlayers of the region 3 of the drift chambers. The hexagonal cells are discussed in more detail in the text. Image taken from [30] with Elsevier permission. . . . . . . . . . . 31

3.16 TOF scintillator shell from one sector. Picture taken from [31] with Elsevier permission. . . . . . . . . . . . . . . 31

3.17 Deconstructed view of one of the electromagnetic modules. Image taken from [32] with Elsevier permission. . . . . . . . . . . 33

3.18 Liquid-deuterium target cell. Picture taken from [34]. . . . . . . 35

4.1 Start Counter offset for $\pi^{-}$particles for an intermediate stage of the Start Counter calibration. The figure shows the subperiod division, and the runs that were chosen to be golden runs. . . . . 40 
4.2 Proton identification. Top left figure is the $\Delta \beta$ vs p plot. The events between the red curves, are considered $\pi^{+}$. The two right plots show the fit for the central region for $\mu+3 \sigma$ (top) and $\mu-$ $3 \sigma$ (bottom), where $\mu$ and $\sigma$ are respectively the mean and the standard deviation of a Gaussian fit over each momentum bin. The left bottom represents $\mu$ for each momentum bin. . . . . . .

4.3 $\pi^{-}$identification. Top left figure is the $\Delta \beta$ vs p plot. The events between the red curves, are considered $\pi^{-}$. The two right plots show the fit for the central region for $\mu+3 \sigma$ (top) and $\mu-3 \sigma$ (bottom), where $\mu$ and $\sigma$ are respectively the mean and the standard deviation of a Gaussian fit over each momentum bin. The left bottom is the mean value for each momentum bin. . . . . .

$4.4 \pi^{+}$identification. Top left figure is the $\Delta \beta$ vs p plot. The events between the red curves, are considered $\pi^{+}$. The two right plots show the fit for the central region for $\mu+3 \sigma$ (top) and $\mu-3 \sigma$ (bottom), where $\mu$ and $\sigma$ are respectively the mean and the standard deviation of a Gaussian fit over each momentum bin. The left bottom represents $\mu$ for each momentum bin. . . . . . . . .

4.5 Rest mass squared distribution for positive particles. In red the distribution before $\Delta \beta$ cut and in blue after the $\Delta \beta$ cut. For further explanation refer to the text. . . . . . . . . . . . . 48

4.6 Rest mass squared distribution for negative particles. In red the distribution before $\Delta \beta$ cut and in blue after the $\Delta \beta$ cut. For further explanation refer to the text. . . . . . . . . . . . . 48 
4.7 $\beta_{\gamma_{1}}$. The events in blue are those that are considered photons, while the distribution in red is cut out. . . . . . . . . . . . . . . 49

4.8 $\beta_{\gamma_{2}}$. The events in blue are those that are considered photons, while the distribution in red is cut out. . . . . . . . . . . . . 49

4.9 Number of good photons per event. . . . . . . . . . . . . . 50

$4.10 \Delta t_{\gamma \pi^{-}}$as a function of the $\pi^{-}$momentum. The RF structure every $2 \mathrm{~ns}$ is clear in the figure. . . . . . . . . . . . . . . 52

$4.11 \Delta t_{\gamma \pi^{-}}$as a function of the $\pi^{-}$momentum after cuts. . . . . . . . 52

4.12 Number of good photons per event that are in the same RF bucket, that is, $|\Delta t|<1$ ns. . . . . . . . . . . . . . . . 53

$4.132-\mathrm{D}$ plot of $\Delta t_{p \pi^{-}}$vs $\Delta t_{\pi^{+} \pi^{-}}$. The red lines represent the cut

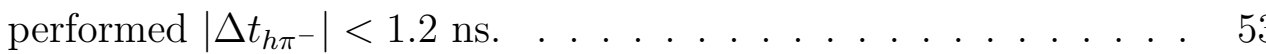

$4.14 z$ coordinate, with respect to the center of CLAS, of the event vertex as found in the MVRT bank. The vast majority of the events are produced within the nominal values of the target. The events in red are those that are cut out of the data sample. . . . 55

$4.15 y$ vs. $x$ coordinate for the primary vertex from MVRT bank. No cut was performed for these quantities. . . . . . . . . . . 55

4.16 Invariant mass squared of the two photons. The red lines mark the cut. The green line indicates the position of the nominal value of the $m_{\pi^{0}}^{2}=0.01822 \mathrm{GeV}^{2} / \mathrm{c}^{4} \ldots \ldots \ldots$. . . . . . . . 56

4.17 Relative momentum difference after energy loss $\Delta p / p_{\text {meas }}$ for the proton after particle identification cuts. . . . . . . . . . . . 58

4.18 Relative momentum difference after energy loss $\Delta p / p_{\text {meas }}$ for the tracks identified as $\pi^{+}$after particle identification cuts. . . . . . . 58 
4.19 Relative momentum difference after energy loss $\Delta p / p_{\text {meas }}$ for the tracks identified as $\pi^{-}$after particle identification cuts. . . . . . . 59

4.20 Relative momentum difference after momentum correction $\Delta p / p_{\text {meas }}$ for the tracks identified as protons. . . . . . . . . . . . . 60

4.21 Relative momentum difference after momentum correction $\Delta p / p_{\text {meas }}$ for the tracks identified as $\pi^{+} \ldots \ldots . . . . . . . . . .660$

4.22 Relative momentum difference after momentum correction $\Delta p / p_{\text {meas }}$ for the tracks identified as $\pi^{-}$. . . . . . . . . . . . . . . . 61

4.23 Tagger correction accounting for the gravitational sag. . . . . . . 61

4.24 Two-Gaussian fit to the projection of the invariant mass squared of the three pions for different missing mass squared bins. The red lines show the $3 \sigma$ cut. . . . . . . . . . . . . . . 64

$4.253 \sigma$ cut around the centroid. In the two bottom plots, it is possible to see the 2nd degree polynomial fit to the $\mu \pm 3 \sigma . \ldots 65$

4.26 Invariant mass squared of the three pions $m^{2}\left(\pi^{+} \pi^{-} \pi^{0}\right)$ vs. the missing mass squared $m_{X}^{2}\left(\gamma d \rightarrow p \pi^{+} \pi^{-} \pi^{0} X\right)$ before (left) and after the cut (right). This is an example for the energy set with coherent

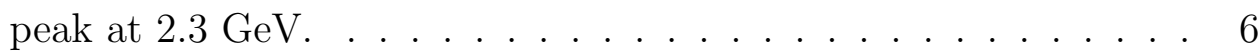


5.1 Schematic representation of the reaction $\gamma p \rightarrow p \omega$ in the centerof-mass frame. The blue rectangle represents the decay plane, and its normal unitary vector is defined by $\hat{n}=\frac{\hat{z} \times p_{C M}^{\omega}}{\left|\hat{z} \times p_{C M}^{\omega}\right|}$. The orange rectangle represents the polarization plane for the incoming photon. The angle between the two planes is denoted as $\beta$. Finaly, the $\theta_{\mathrm{CM}}$ is the angle between the proton and the $\omega$ meson in the center-of-mass frame. The $z$-axis is the direction of the incoming photon. ...................... 67

5.2 Difference between the $\cos \theta$ generated with the Monte Carlo, and the $\cos \theta$ "detected" as a function of $\cos \theta_{\mathrm{CM}}$. The top plot takes the information of the incoming particles assuming the proton at rest. The bottom plot takes the information from the final-state particles without any assumption. . . . . . . . . . . . 68

5.3 Typical photon polarization (top) and corresponding enhancement distribution (bottom). . . . . . . . . . . . . . 74

5.4 Example of fit for $1.7<E_{\gamma}<1.8 \mathrm{GeV}$. . . . . . . . . . . . . 75

5.5 Fit for the asymmetry extraction. The red dots and fit are taken before the dilution factor correction is applied. The blue dots and fit are calculated after the dilution factor is estimated by integrating the histogram. The green dots and fit are calculated after the dilution factor is estimated by integrating a double-Gaussian-fit model to the background. . . . . . . . . . . . . . . 78

5.6 Fitting the modulation of the ratio $Y^{*}$ for different kinematic bins in $\cos \theta_{\mathrm{CM}} \ldots \ldots \ldots \ldots$. . . . . . . . . . . . . . 80 
5.7 Result for Beam Spin Asymmetry for $1.1<E_{\gamma}<2.3 \mathrm{GeV}$ in energy bins of $\Delta E_{\gamma}=200 \mathrm{MeV}$. The blue triangles represent this work (quasi-free proton), the red circles represent the GRAAL 2015 [17] results for the quasi-free reaction. The green squares are the results from [14] for the free-proton channel. The blue line represents the Bonn-Gatchina prediction after including the $\omega$ photoproduction data from CLAS 2017 [14] in their fit. . . . . . . 81

6.1 Normalized residuals distribution for each energy bin. . . . . . . 83

6.2 $\Delta \Sigma$ as a function of $\cos \theta_{C M} . \Sigma^{*}$ is calculated using a broader cut on the neutral particle identification $\left(\beta_{n}<0.9\right)$. . . . . . . .

6.3 $\Delta \Sigma$ as a function of $\cos \theta_{C M} . \quad \Sigma^{*}$ is calculated using a broader cut on the incoming photon coincidence time with $\pi^{-}\left(\Delta t_{\gamma \pi^{-}} \leq\right.$ $1.2 \mathrm{~ns}) . \ldots \ldots \ldots \ldots \ldots$

6.4 $\Delta \Sigma$ as a function of $\cos \theta_{C M} . \Sigma^{*}$ is calculated using a tighter cut on the proton and $\pi^{+}$coincidence time with $\pi^{-}\left(\Delta t_{h \pi^{-}} \leq 1.0 \mathrm{~ns}\right)$.

6.5 $\Delta \Sigma$ as a function of $\cos \theta_{C M} . \Sigma^{*}$ is calculated using a broader cut on the $z$ coordinate of the primary vertex $(-40 \leq z \leq 0 \mathrm{~cm}) . \quad . \quad$. 89

6.6 $\Delta \Sigma$ as a function of $\cos \theta_{C M} . \Sigma^{*}$ is calculated using a tighter cut on the missing momentum $\left(P_{X}<0.15 \mathrm{GeV} / \mathrm{c}\right)$. . . . . . . . . . 90

6.7 $\Delta \Sigma$ as a function of $\cos \theta_{C M} . \Sigma^{*}$ is calculated using a tighter $2-\sigma$ cut on the calculation of the dilution factor. . . . . . . . . . . 90 


\section{List of Tables}

5.1 Polarization ratio and polarization average for all energy subsets with different coherent peak. . . . . . . . . . . . . . . 74

5.2 Calculated values for the flux ratio $F_{R} \ldots \ldots \ldots 76$

5.3 Dilution factor for each energy subset for PARA and PERP polarization. HISTO and FIT stand for integration over the histogram, and integration over the double-Gaussian model. . . . . . . . . . 77

6.1 Calculated values for the systematic uncertainty related to the flux ratio $F_{\mathrm{R}} \ldots \ldots \ldots \ldots \ldots$

6.2 Summary of the estimated values for the systematic uncertainties related to different sources. As extensively explained in this chapter, the estimated systematic uncertainties related with the kinematical cuts are averages of the absolute difference of the parameter $\Sigma$ and its variation. . . . . . . . . . . . . . . 92 


\section{Abstract}

Quantum chromodynamics (QCD) is the fundamental theory underlying the strong interaction. Great effort has been dedicated to explain hadron interactions and the hadron composition in terms of the QCD degrees of freedom (quarks and gluons). However, this task has been largely complicated by confinement, an intrinsic feature of QCD itself, which prevents us from a direct experimental study of the dynamics of quarks and gluons. Instead, QCD-inspired models have been developed to explain the hadron spectrum, and while there has been success in explaining important features of it, many of the states that have been predicted by these models have not been found experimentally.

Furthermore, the hadronic spectrum is much more complicated to analyze than the atomic one because of a high number of excited states of the nucleon which have large widths that cause resonances to overlap. Also, these resonances may decay into a multitude of decay channels involving either mesons or baryons. Additionally, we will need measurements of observables, coming from polarized beam and polarized target experiments, to obtain a complete measurement of all the helicity amplitudes.

My current research aims at contributing to a larger experimental program that seeks to shed light on the evolving status of the proton spectrum. I focus on the photoproduction of $\omega$ mesons off the bound proton, which is of great interest 
in providing information about $N^{*}$ resonances as $\omega$ is an isospin filter. I have extracted a preliminary quasi-free $\vec{\gamma} d \rightarrow \omega p(n)$ photon beam asymmetry polarization observable from CLAS data. The $\omega$ meson was identified through its charged decay $\omega \rightarrow \pi^{+} \pi^{-} \pi^{0}$ where the $\pi^{0}$ subsequently decays in two photons. The data were taken during the E06-103 experiment with the CLAS detector in Hall B at the Thomas Jefferson National Accelerator Laboratory (JLab). The experiment used the Hall-B Coherent Bremsstrahlung Facility to provide a high quality beam of linearly-polarized photons in the energy range from 1.1 to $2.3 \mathrm{GeV}$. 


\section{Chapter 1}

\section{Hadron Spectrum}

\section{$1.1 \quad$ Baryon Spectroscopy}

Baryons are strongly interacting fermions that have a baryon number $B=1$, consistent with a $q q q$ configuration. Light baryons that consist only of $u$ and $d$

quarks, with isospin $I=\frac{1}{2}$, are called nucleons, and those with isospin $I=\frac{3}{2}$ are called $\Delta$ resonances. The study of the nucleon spectrum is key for understanding the internal dynamics of the nucleon as a whole. It further provides a means to test the predictions of any number of theoretical approaches used in modeling quantum chromodynamics (QCD).

Our understanding of the nucleon as a bound state of quarks and gluons (QCD degrees of freedom) is still a great challenge for the hadron physics community. It is of fundamental importance to study simultaneously the structure and the properties of the nucleon in the ground state, along with studying the excited states of baryons, which are also known as baryon resonances. On one hand, deep inelastic scattering experiments are used to study the properties of the baryon's constituent's ground states, and on the other hand, we have the spectroscopy 
experiments, which can shed light on the collective degrees of freedom by probing the nucleon as a whole.

The paradigm of what can be learned by hadron spectroscopy studies was taught to us by atomic spectroscopy. In the latter case, we study an electricallyneutral object (the atom) composed of electrically-charged components that interact via the electromagnetic force that is governed by quantum electrodynamics (QED). Similarly, for hadron spectroscopy, we study a color-singlet object built by color charged components that follow QCD rules.

In this energy regime (the confinement region), the hadron spectrum cannot be calculated using standard perturbation theory. Instead, different approaches have been developed to predict the hadron spectrum. The phenomenological as well as QCD-inspired models [1] have given us most of the theoretical insight. Within these models, the constituent quark models take the QCD Lagrangian and replace the valence quark ${ }^{1}$ with an effective quark or constituent quark. The interaction of these constituent quarks is mediated through potentials that force the correct behavior at large and small distances, i.e., asymptotic freedom at large energies and quark confinement at low energies. Lattice QCD (see for instance [2]) has started to complement this information with numerical solutions of QCD. Lattice QCD aims to solve the exact QCD Lagrangian by assuming a discrete space-time.

While there has been success in explaining important features of the hadron spectrum, many of the excited states that have been predicted have not been found experimentally. The main issue behind the problem of the "missing baryon resonances" is that we do not have a clear understanding of the number of the effective degrees of freedom.

\footnotetext{
${ }^{1}$ The quarks found in the standard model.
} 
Instead of considering three constituent quarks, the degrees of freedom are reduced by assuming the baryons to be a quark-diquark bound system [1]. This assumption dramatically reduces the number of excited states in the baryon spectrum [3]. However, the diquark model still predicts a richer spectrum than that of the one observed experimentally. One other possibility in explaining the missing baryon resonances issue may well be experimental. The data obtained in the early developments of nucleon spectroscopy came from $\pi-N$ elastic scattering. Some of the missing resonances might couple weakly to this channel, therefore escaping identification. The extraction of the resonant information has both experimental and theoretical challenges.

From the experimental point of view, we come into several challenges. The hadronic spectrum is much more complicated to analyze than the atomic one because of a high number of excited states of the nucleon which have large widths that cause resonances to overlap. Also, these resonances may decay into a multitude of decay channels involving either mesons or baryons; an example of this can be seen in the table shown in figure 1.1. It is important to note the large cross section that $\omega$ has for $E_{\gamma}>1.7 \mathrm{GeV}$, which corresponds to the part of the hadron spectrum that is least understood.

In order to identify a state in the hadron spectrum (tagged by spin, parity, and isospin $)^{2}$, the observables obtained for different channels have to be decomposed into partial waves to allow for extracting these quantum numbers. However, the extraction of these quantum numbers is not unambiguous: to isolate resonant states from the nonresonant background states, we need to use reaction

\footnotetext{
${ }^{2}$ The notation used in the past, when most of the information came from elastic $\pi \mathrm{N}$ scattering, was to label the resonances with $L_{2 I, 2 J}$ (For example $\mathrm{N}(1680) F_{15}$ ). Now that the information also comes from $\gamma \mathrm{N}$ experiments, this notation has been replaced with the spin-parity $J^{P}$ of the state (For example $\mathrm{N}\left(16805 / 2^{+}\right)$.
} 


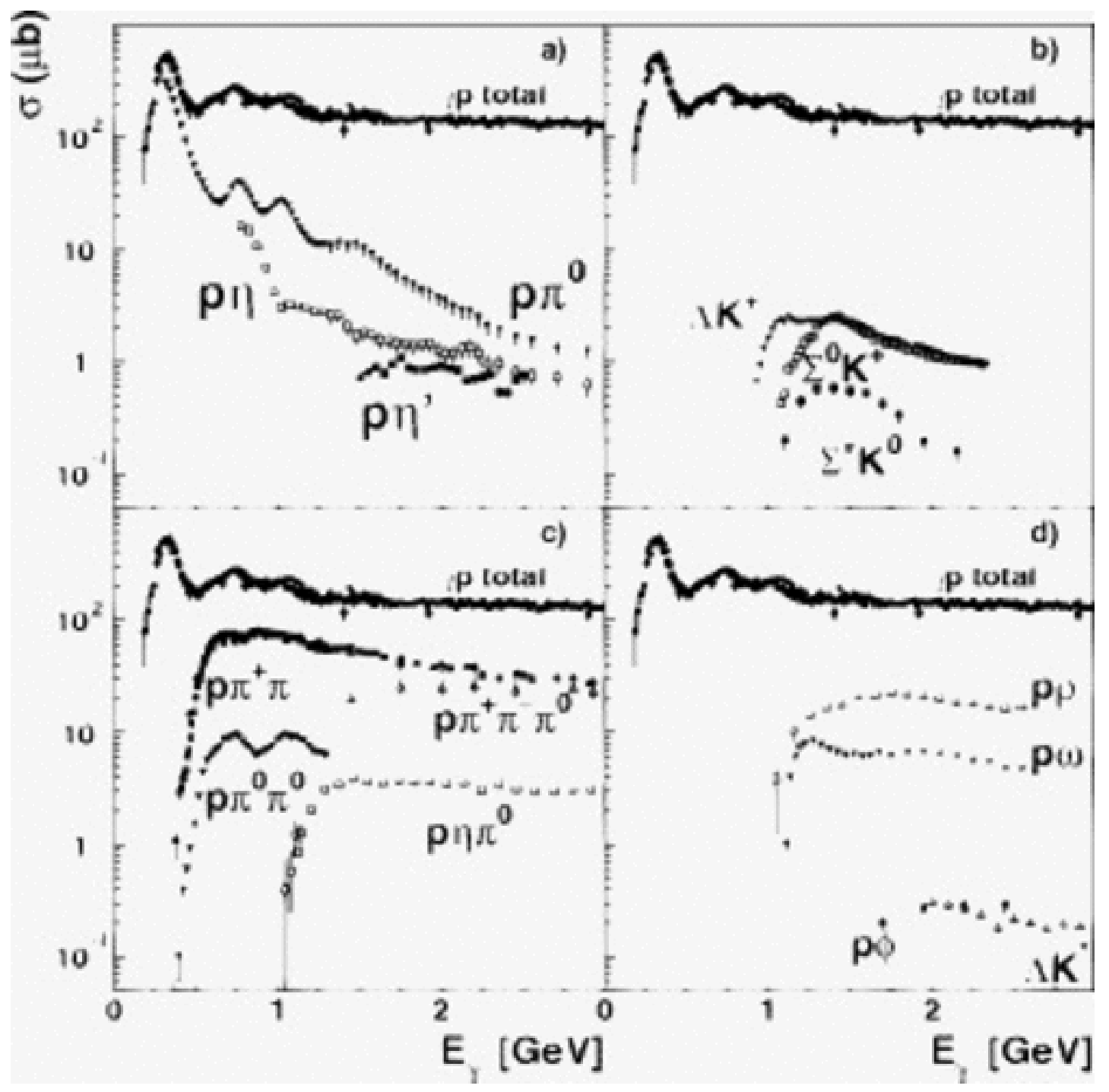

Fig. 1.1. Total photo-absorption cross section and exclusive cross sections for different channels as a function of the photon energy.

models. This model dependency, which can vary among different collaborations performing the partial wave analysis, results in several calculated nucleon resonance spectra. That is to say, some excited baryons are included and other baryon resonances are not.

The information from different groups is collected in the particle data group 
report and each possible resonant state is assigned with a number of stars ranging from $4(* * * *)$ to $1(*)$. An overall status of $* * * *$ or $* * *$ is considered to be established resonances; these come from data sets that include both differential cross sections and polarization observables and are confirmed by an independent analysis. A status of $*$ and $* *$ is given to all other signals. The table shown in figure 1.2 presents the Breit-Wigner masses and widths of $\mathrm{N}$ resonances [4] calculated from different partial wave analysis groups. Note that the region from $1.8-2.2 \mathrm{GeV}$ is poorly understood and needs to be studied in more detail.

\begin{tabular}{|c|c|c|c|c|c|c|c|}
\hline Resonance & $\begin{array}{c}\text { Our } \\
\text { estimate }\end{array}$ & $\begin{array}{l}\text { Our } \\
\text { rating }\end{array}$ & $\mathrm{KH}$ & $\mathrm{CM}$ & Kent & GWU & $\mathrm{BnGa}$ \\
\hline$N_{1 / 2}+(1440)$ & $1450 \pm 32 ; 300 \pm 100$ & $* * * *$ & $1410 \pm 12 ; 135 \pm 10$ & $1440 \pm 30 ; 340 \pm 70$ & $1462 \pm 10 ; 391 \pm 34$ & $1485 \pm 1 ; 284 \pm 18$ & $1440 \pm 12 ; 335 \pm 50$ \\
\hline$N_{3 / 2^{-}}(1520)$ & $1522 \pm 4 ; 115 \pm 10$ & $* * * *$ & $1519 \pm 4 ; 114 \pm 7$ & $1525 \pm 10 ; 120 \pm 15$ & $1524 \pm 4 ; 124 \pm 8$ & $1516 \pm 1 ; 99 \pm 3$ & $1524 \pm 4 ; 117 \pm 6$ \\
\hline$N_{1 / 2}-(1535)$ & $1538 \pm 10 ; 175 \pm 45$ & $* * * *$ & $1526 \pm 7 ; 120 \pm 20$ & $1550 \pm 40 ; 240 \pm 80$ & $1534 \pm 7 ; 151 \pm 27$ & $1547 \pm 1 ; 188 \pm 4$ & $1535 \pm 20 ; 170 \pm 35$ \\
\hline$N_{1 / 2^{-}}(1650)$ & $1660 \pm 18 ; 165 \pm 25$ & $* * * *$ & $1670 \pm 8 ; 180 \pm 20$ & $1650 \pm 30 ; 150 \pm 40$ & $1659 \pm 9 ; 170 \pm 12$ & $1635 \pm 1 ; 115 \pm 3$ & $1680 \pm 40 ; 170 \pm 45$ \\
\hline$N_{5 / 2-}(1675)$ & $1675 \pm 5 ; 153 \pm 22$ & $* * * *$ & $1679 \pm 8 ; 120 \pm 15$ & $1675 \pm 10 ; 160 \pm 20$ & $1676 \pm 2 ; 159 \pm 7$ & $1674 \pm 1 ; 147 \pm 1$ & $1678 \pm 5 ; 177 \pm 15$ \\
\hline$N_{5 / 2}+(1680)$ & $1683 \pm 3 ; 126 \pm 9$ & $* * * *$ & $1684 \pm 3 ; 128 \pm 8$ & $1680 \pm 10 ; 120 \pm 10$ & $1684 \pm 4 ; 139 \pm 8$ & $1680 \pm 1 ; 128 \pm 1$ & $1685 \pm 5 ; 117 \pm 12$ \\
\hline$N_{3 / 2^{-}}(1700)$ & $1725 \pm 50 ; 190 \pm 110$ & $* * *$ & $1731 \pm 15 ; 110 \pm 30$ & $1675 \pm 25 ; 90 \pm 40$ & $1737 \pm 44 ; 250 \pm 230$ & - & $1730 \pm 40 ; 310 \pm 60$ \\
\hline$N_{1 / 2}+(1710)$ & $1713 \pm 12 ; 220 \pm 180$ & *** & $1723 \pm 9 ; 120 \pm 15$ & $1700 \pm 50 ; 90 \pm 30$ & $1717 \pm 28 ; 480 \pm 330$ & - & $1725 \pm 25 ; 200 \pm 35$ \\
\hline$N_{3 / 2+}(1720)$ & $1730 \pm 30 ; 320 \pm 210$ & $* * * *$ & $1710 \pm 20 ; 190 \pm 30$ & $1700 \pm 50 ; 125 \pm 70$ & $1717 \pm 31 ; 380 \pm 180$ & $1750 \pm 5 ; 256 \pm 22$ & $1770 \pm 100 ; 650 \pm 120$ \\
\hline$N_{3 / 2^{-}}(1860)$ & $1850 \pm 40 ; 260 \pm 170$ & ** & - & $1880 \pm 100 ; 180 \pm 60$ & $1804 \pm 55 ; 450 \pm 185$ & - & $1870 \pm 25 ; 150 \pm 40$ \\
\hline$N_{5 / 2}+(1870)$ & $1880 \pm 40 ; 270 \pm 180$ & $\overline{* *}$ & $1882 \pm 10 ; 95 \pm 20$ & - & $1903 \pm 87 ; 490 \pm 310$ & $1818 ; 118$ & $1910 \pm 50 ; 360 \pm 80$ \\
\hline$N_{1 / 2}+(1880)$ & $1890 \pm 50 ; 210 \pm 100$ & * & - & - & $1885 \pm 30 ; 113 \pm 44$ & - & $1900 \pm 30 ; 300 \pm 40$ \\
\hline$N_{3 / 2+}(1900)$ & $1940 \pm 50 ; 340 \pm 150$ & * & - & - & $1879 \pm 17 ; 498 \pm 78$ & - & $1960 \pm 30 ; 185 \pm 40$ \\
\hline$N_{1 / 2^{-}}(1905)$ & $1905 \pm 50 ; 250 \pm 150$ & $*$ & $1880 \pm 20 ; 95 \pm 30$ & - & $1928 \pm 59 ; 414 \pm 157$ & - & - \\
\hline$N_{7 / 2+}(1990)$ & $2020 \pm 60 ; 410 \pm 110$ & $* *$ & $2005 \pm 150 ; 350 \pm 100$ & $1970 \pm 50 ; 350 \pm 120$ & $2086 \pm 28 ; 535 \pm 120$ & - & - \\
\hline$N_{3 / 2^{-}}(2080)$ & $2100 \pm 55 ; 310 \pm 110$ & $\overline{* *}$ & $2080 \pm 20 ; 265 \pm 40$ & $2060 \pm 80 ; 300 \pm 100$ & - & - & $2160 \pm 35 ; 370 \pm 50$ \\
\hline$N_{1 / 2^{-}}(2090)$ & & & & $2180 \pm 80 ; 350 \pm 100$ & - & - & - \\
\hline$N_{1 / 2}+(2100)$ & $2090 \pm 100 ; 230 \pm 200$ & * & $2050 \pm 20 ; 200 \pm 30$ & $2125 \pm 75 ; 260 \pm 100$ & - & - & - \\
\hline \multirow[t]{2}{*}{$N_{5 / 2}-(2200)$} & $2160 \pm 85 ; 350 \pm 50$ & *** & $2228 \pm 30 ; 310 \pm 50$ & $2180 \pm 80 ; 400 \pm 100$ & - & - & $2065 \pm 25 ; 340 \pm 40$ \\
\hline & & & KH & $\mathrm{CM}$ & Kent & GWU & Hendry \\
\hline$N_{7 / 2^{-}}(2190)$ & $2150 \pm 30 ; 440 \pm 110$ & $* * * *$ & $2140 \pm 12 ; 390 \pm 30$ & $2200 \pm 70 ; 500 \pm 150$ & $2127 \pm 9 ; 550 \pm 50$ & $2152 \pm 2 ; 484 \pm 13$ & $2140 \pm 40 ; 270 \pm 50$ \\
\hline$N_{9 / 2+}(2220)$ & $2260 \pm 60 ; 490 \pm 115$ & $* * * *$ & $2205 \pm 10 ; 365 \pm 30$ & $2230 \pm 80 ; 500 \pm 150$ & - & $2316 \pm 3 ; 633 \pm 17$ & $2300 \pm 100 ; 450 \pm 150$ \\
\hline$N_{9 / 2^{-}}(2250)$ & $2255 \pm 50 ; 420 \pm 150$ & $* * * *$ & $2268 \pm 15 ; 300 \pm 40$ & $2250 \pm 80 ; 400 \pm 120$ & - & $2302 \pm 6 ; 628 \pm 28$ & $2200 \pm 100 ; 350 \pm 100$ \\
\hline$N_{11 / 2-}(2600)$ & $2630 \pm 120 ; 650 \pm 250$ & $* *$ & $2577 \pm 50 ; 400 \pm 100$ & - & - & - & $2700 \pm 100 ; 900 \pm 100$ \\
\hline$N_{13 / 2}+(2700)$ & $2800 \pm 160 ; 600 \pm 300$ & *** & $2612 \pm 45 ; 350 \pm 50$ & - & - & - & $3000 \pm 100 ; 900 \pm 150$ \\
\hline
\end{tabular}

Fig. 1.2. Breit-Wigner masses and widths of $N^{*}$ resonances. Table taken from [4] with permission.

Furthermore, for processes with spin, we will need measurement of observables coming from a polarized beam and a polarized target towards obtaining a complete measurement of all the helicity amplitudes. 


\subsubsection{Photoproduction of Vector Mesons}

The quantum numbers associated with the photon are the same as the unflavored vector meson. For energies close to the threshold, which is the energy range studied in this thesis, three major mechanisms contribute to the cross section:

- Natural parity $t$-channel exchange or pomeron exchange,

- $t$-channel pion exchange,

- s-channel resonance excitation.

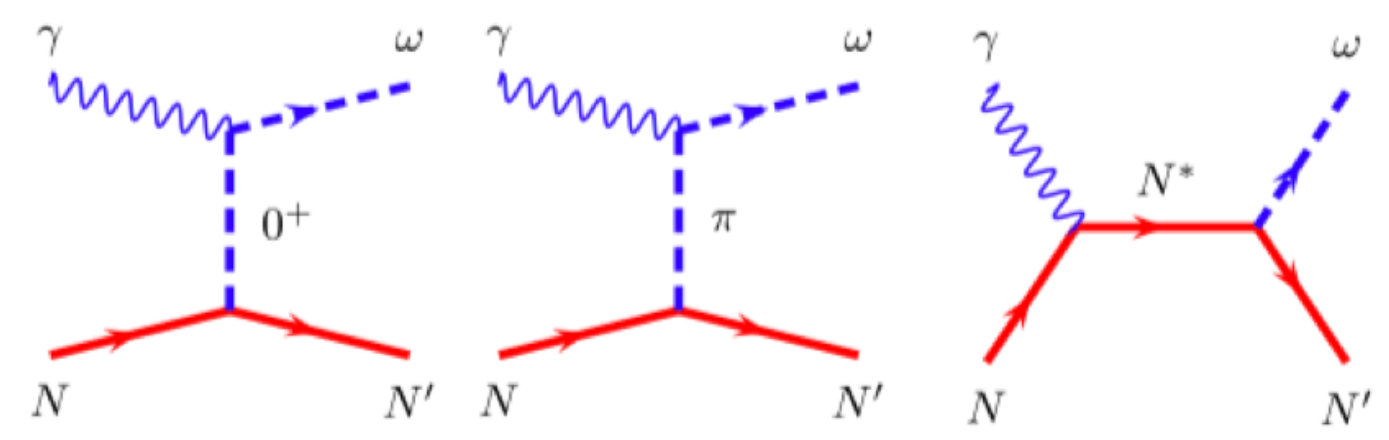

Fig. 1.3. Main mechanisms in the $\omega$ photoproduction using real photons. From left to right: $t$-channel pomeron exchange, $t$-channel pion exchange, $s$-channel $N^{*}$ resonance.

For energies near threshold, the leading term in the cross section for the photoproduction of vector mesons using real photon probes is natural parity exchange. This is evidenced by the typical diffractive behavior through exponential fall-off of the cross section. The $t$-channel pion exchange is expected to be large because of a large $\omega \rightarrow \pi^{0} \gamma$ coupling. Finally, the most relevant in the context of baryon spectroscopy, which is the main focus of this thesis, is the $s$-channel contribution. In the next chapter, we will discuss not only the previous experimental 
data, but also their interpretation and the $N^{*}$ contributions that these data have suggested. 


\section{Chapter 2}

\section{Photoproduction of $\omega$ Mesons}

The $\omega$ meson is the channel that we take under consideration for this work. It is a vector meson with quantum numbers $I^{G}\left(J^{P C}\right)=0^{-}\left(1^{--}\right)$. As reported in [5], the weighted average of the mass is $782.65 \pm 0.12 \mathrm{MeV} / \mathrm{c}^{2}$, with an average width of $8.49 \pm 0.08 \mathrm{MeV} / \mathrm{c}^{2}$. The three main decay modes for this meson are:

- $\omega \rightarrow \pi^{+} \pi^{-} \pi^{0}$ with a branching ratio of $89.2 \pm 0.7 \%$,

- $\omega \rightarrow \gamma \pi^{0}$ with a branching ratio of $8.40 \pm 0.22 \%$ and

- $\omega \rightarrow \pi^{+} \pi^{-}$with a branching ratio of $1.53 \pm_{0.13}^{0.11} \%$.

I shall focus on the calculation of the beam asymmetry for the photoproduction of $\omega$ mesons off the bound proton inside deuterium using a linearly polarized photon beam. This decay channel is of great interest in providing information about $N^{*}$ resonances as $\omega$ is an isospin filter because $\omega N$ states can only be produced by $N^{*}$ states with isospin $I=1 / 2$ and not by $\Delta^{*}$ states with isospin $I=3 / 2$. Furthermore, since its threshold is above the $\pi$ and $\eta$ photoproduction thresholds, the information obtained from the $\omega$ meson channel can give infor- 
mation of higher mass resonances (i.e., the region where the proton spectrum is less understood).

\subsection{Vector Meson Photoproduction}

In the reaction $\gamma p \rightarrow p \omega$ we can have a total of 24 helicity amplitudes since we can have three helicities for the vector meson and two helicities for the beam, the target, and the recoil proton $(3 \times 2 \times 2 \times 2=24)$. There are 12 relations between these amplitudes and one can reduce the number of independent complex helicity amplitudes to 12 [6]. It is important to notice, as discussed in the previous section, that the scattering amplitudes of different processes are very entangled, thereby forcing us to require not only the amplitude but also the phase of the scattering amplitudes. As a result, a total of 23 independent measurements have to be measured to have a "complete" description of the vector meson. Although such a complete experiment is not currently possible, polarization observables will constrain the bilinear combinations of the helicity amplitudes and thereby provide far more information that just from unpolarized cross sections. There is another set of observables defined in the 1970s by Schilling [7] termed the Spin Density Matrix Elements (SDMEs), which can be written in terms of a bilinear combination of the helicity amplitudes. These observables are tied to the polarization of the vector meson by understanding the decay distribution of the $\omega$ meson. 


\subsection{Previous Measurements for $\omega$ Photoproduction}

The study of $\omega$ meson photoproduction has been a subject of study for around 50 years. It is important to note that 20 years ago this topic regained relevance, given the great amount of data collected by modern experimental facilities around the world. For a free proton target, experiments from SLAC and Daresbury in the 1970s [8] and from CLAS in 2003 [9] extracted the unpolarized differential cross section for a kinematic region of $E_{C M} \in \sim[2.5-2.9 \mathrm{GeV}]$. The SLAC data were produced using monochromatic photons from a backscattered laser beam, going into a hydrogen bubble chamber. These two studies show a predominance of a $t$-channel production mechanism for this energy region. For a larger kinematic region including near-threshold energies, the unpolarized differential cross section and the unpolarized SDMEs were extracted by the SAPHIR collaboration in 2003 [10], the CLAS collaboration in 2009 [11, 12], and CBELSA/TAPS in 2015 [13]. Each collaboration agreed on the importance of $s$-channel processes to describe their data. The CLAS collaboration went a step further and performed an independent Partial Wave Analysis (PWA), where several additional resonances were found to contribute to the process. There was good agreement between the SDMEs from the two collaborations, but there is an energy-dependent disagreement in the normalization for the differential cross section; this discrepancy has not been resolved yet.

For polarized observables, the beam asymmetry $\Sigma$ has also been extracted from different experiments. The current published data from CBELSA/TAPS collaboration in 2008 [15], the GRAAL collaboration in 2006 and 2015 [16, 17], and the most recent and more precise data from CLAS in 2017 [14], all show the same general angular dependence (see figure 2.1). This similarity means that the 


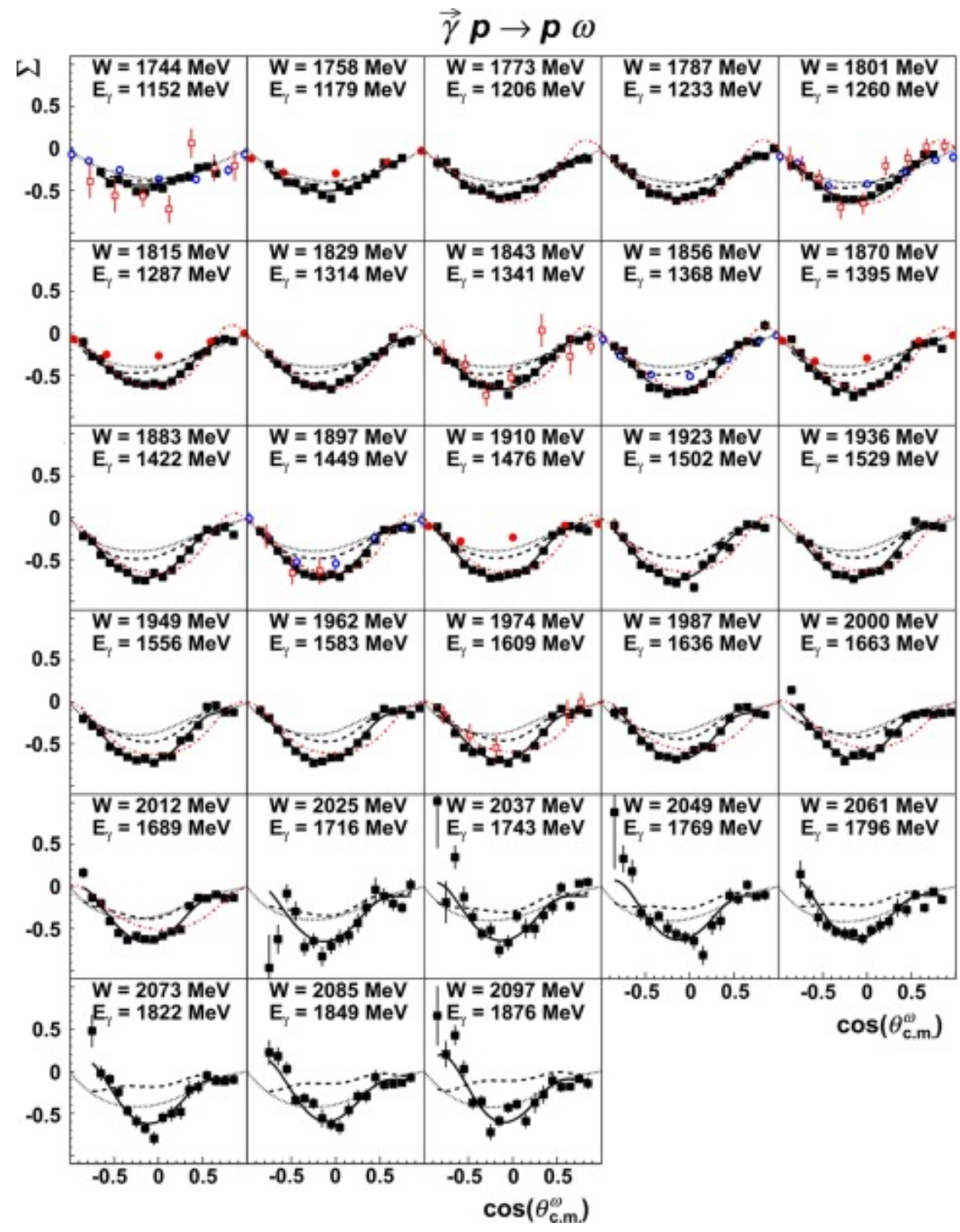

Fig. 2.1. Photon beam asymmetry $\Sigma$ as a function of $\cos \theta_{\mathrm{CM}}$ for the reaction $\vec{\gamma} p \rightarrow \omega p$ for photon energy $E_{\gamma} \in[1.152,1.876] \mathrm{GeV}$ as reported in [14]. The black squares are the result of CLAS 2017 study, it shows only statistical error and not a $6 \%$ systematic uncertainty for each energy bin. The open red squares are the results from the CBELSA/TAPS [15], the blue open open circles and the red solid circles from the GRAAL 2006 [16] and GRAAL 2015 [17] respectively. Figure taken from [14].

shape of the function describing the $\Sigma\left(\cos \theta_{C M}\right)$ for a fixed energy bin is the same. While the data from CBELSA/TAPS and CLAS are in agreement for most of the 
kinematic region, the two GRAAL results do not agree with each other or with the CLAS data. The biggest discrepancy is given for intermediate angles, while for the most forward and backward angles all of the measurements coincide with a value close to zero.

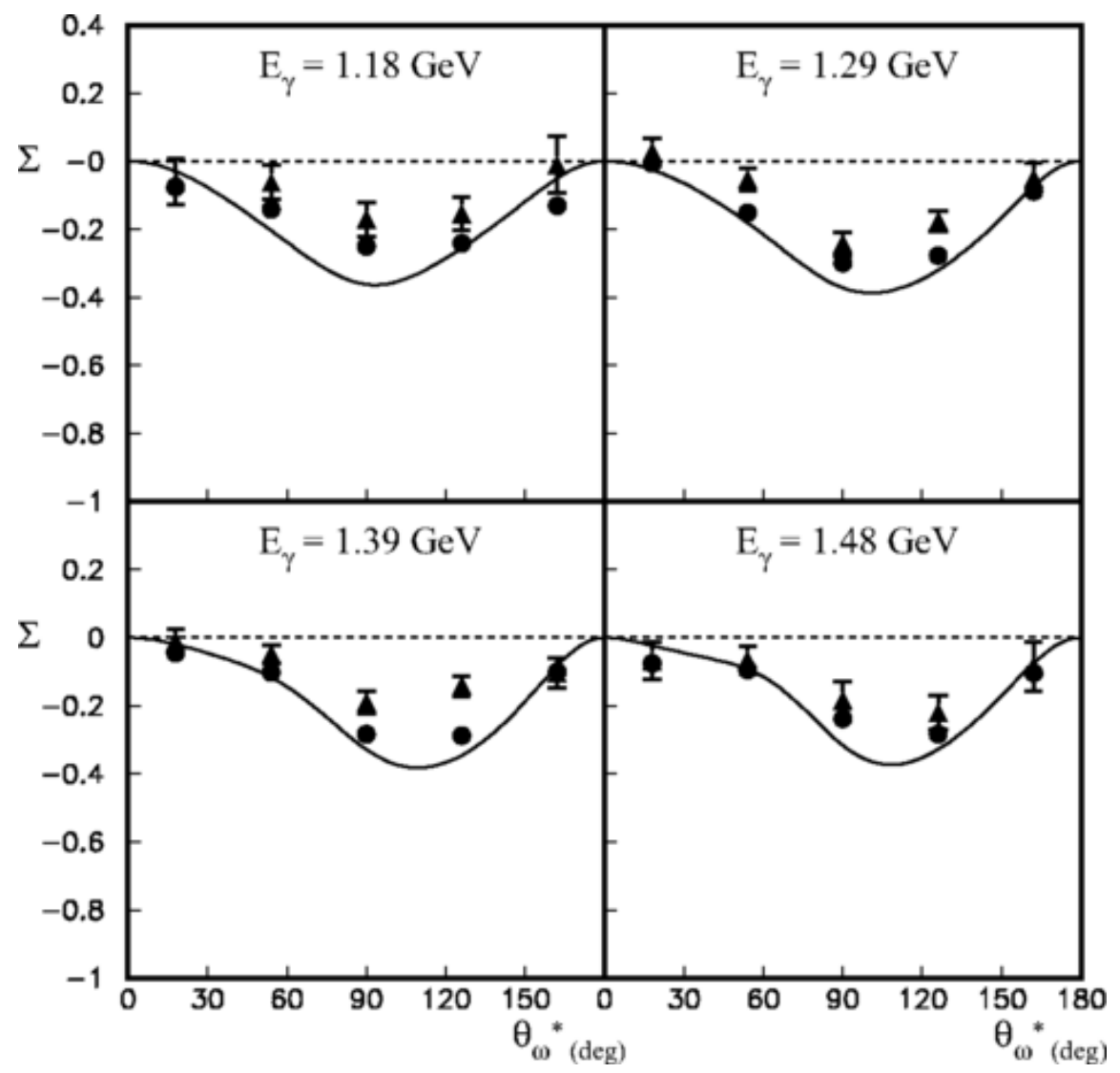

Fig. 2.2. Beam asymmetry $\Sigma$ as a function of $\theta_{C M}$. The full circles represent the free proton channel, the full triangles the quasifree proton in a deuteron target for $E_{\gamma} \in[1.18,1.48] \mathrm{GeV}$. Only statistical uncertainties are shown in the figure and reported in the paper. The lines represent the Zhao model described in [18-20]. Figure taken from [17].

All the measurements described above refer to $\omega$ photo-production off the free proton. Finally, we compare our data with GRAAL [17], as GRAAL is the experiment that has published data from photoproduced omegas from bound 
protons in deuterium. The GRAAL 2013 paper finds that the photon asymmetry observable $\Sigma$ to be the same for both the case of the free proton in hydrogen and the quasi-free bound proton in deuterium (see Figure 2.2). We will note later that the results of this analysis find that $\Sigma_{\text {free }}$ reported from the CLAS collaboration 2017 differs from $\Sigma_{\text {bound }}$ presented in this work. 


\section{Chapter 3}

\section{Experimental Layout}

The Superconducting Radio Frequency (srf) Continuous Electron Beam Accelerator Facility (CEBAF) at the Thomas Jefferson National Accelerator Facility (JLab) is a high-duty factor electron accelerator providing a multi-GeV electron beam (see figure 3.1). The high-current, high-duty-cycle accelerator (section 3.1) allows us to use electromagnetic probes to investigate the internal structure of hadrons and nuclei. To determine an excited hadron reaction exclusively, we need to efficiently detect the multi-particle final state over a large kinematic region. The CEBAF Large Acceptance Spectrometer (CLAS), located in Hall B, is a large acceptance detector capable of precisely detecting charged and neutral particles (see section 3.3). The CLAS detector was designed to be able to operate with

both electron and photon beams. In this document, we describe data produced using a linear polarized photon beam obtained via the coherent bremsstrahlung radiation technique explained in the section 3.2. 


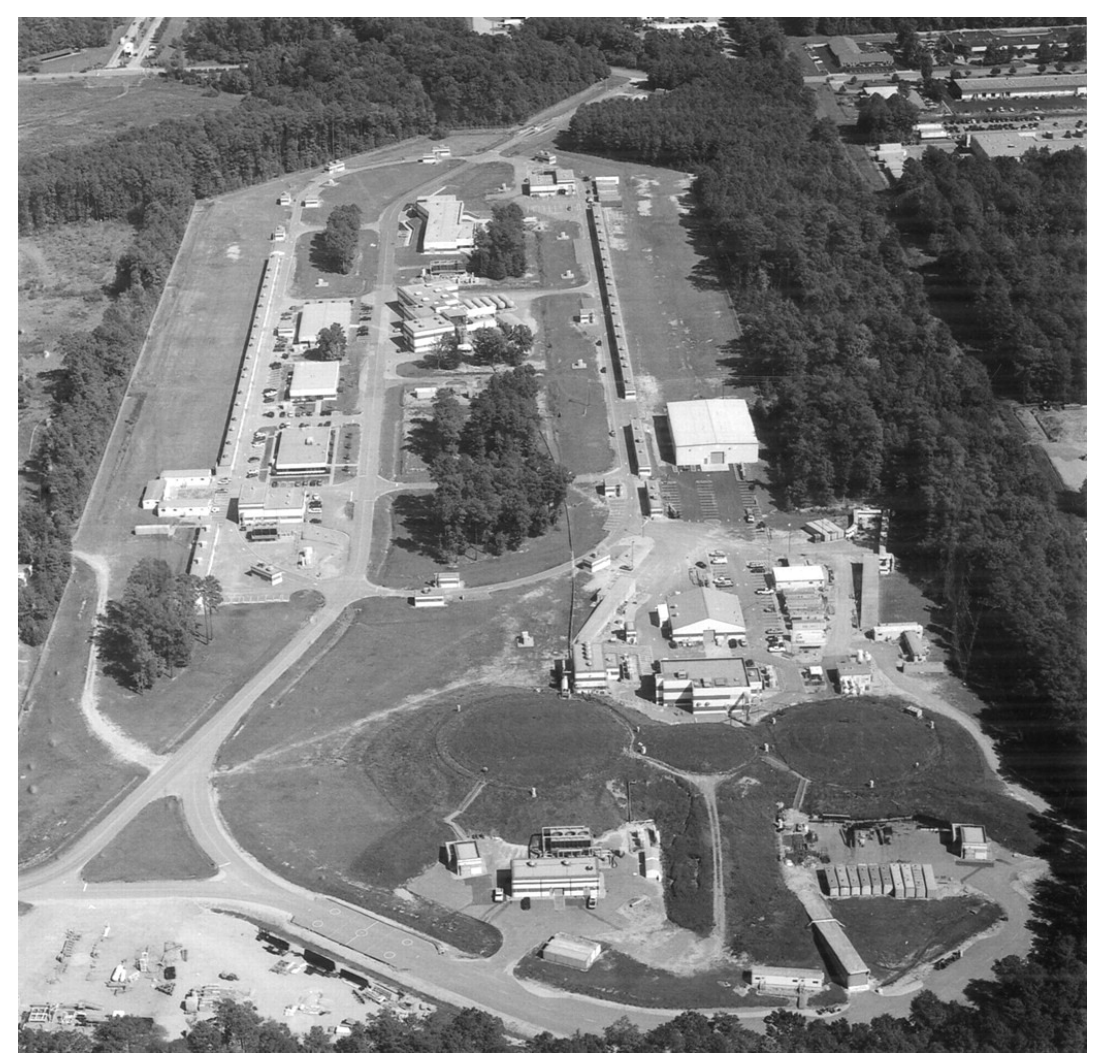

Fig. 3.1. Aerial view of the CEBAF at the Thomas Jefferson National Accelerator Facility (JLab). Picture taken from [21].

\subsection{Electron Beam}

CEBAF can deliver a continuous wave electron beams to three different experimental areas, Halls A, B and C, simultaneously. After the $12 \mathrm{GeV}$ upgrade, a fourth experimental area was added, Hall D. Since the data used in this work were taken before the upgrade, we will only discuss the accelerator composition for energies up to $6 \mathrm{GeV}$. CEBAF racetrack configuration (see figure 3.2) consists of two antiparallel LINear ACcelerator (LINAC) segments (north and south) connected by 9 recirculation beamlines allowing up to 5 passes. The beam currents supplied to Halls A and C range between $1-200 \mu \mathrm{A}$, while for Hall $\mathrm{B}$ the currents range between $1-100 \mathrm{nA}$ [21]. 


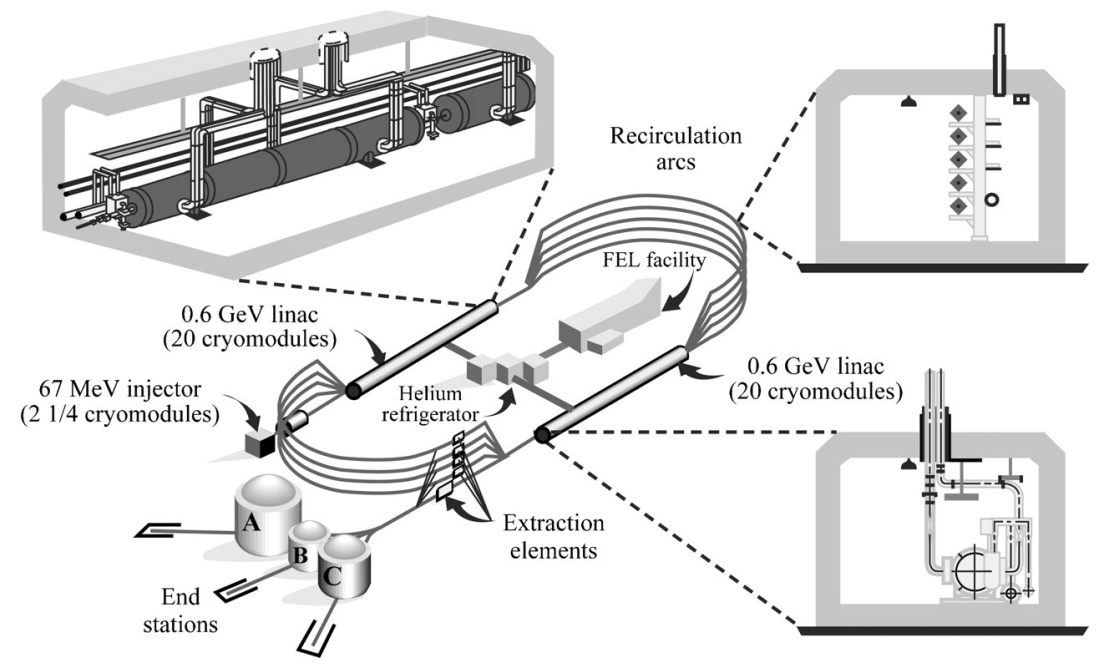

Fig. 3.2. Experimental Layout of the CEBAF accelerator before the $12 \mathrm{GeV}$ upgrade. Picture taken from [22].

Electrons are produced via photoemission using three independent radiofrequency-gain switched lasers directed at a GaAs photocathode. Each electron beam, one for each experimental hall, is generated at a $499 \mathrm{MHz}$ frequency with $120^{\circ}$ phase separation. The three beams are then combined in a $1497 \mathrm{MHz}$ electron bunch train, and the bunch train is accelerated to $67 \mathrm{MeV}$. The combined electron beam is then injected into one of the LINACs. Each LINAC contains 20 cryomodules, each one consisting of 8 superconducting niobium cavities. The cavities inside the cryomodules were immersed in liquid helium at temperatures of $2 \mathrm{~K}$ to ensure superconducting conditions. Microwaves are directed into these cavities causing a charge gradient along the beamline (see figure 3.3). The microwaves must cycle the position of the charged regions 1.5 billion times per second to ensure that the high-speed electrons from the beam have a positively charged area ahead of them and a negatively charged area behind them (see figure $3.4)$.

Every time the beam passes through one of the LINACs, its energy will be 


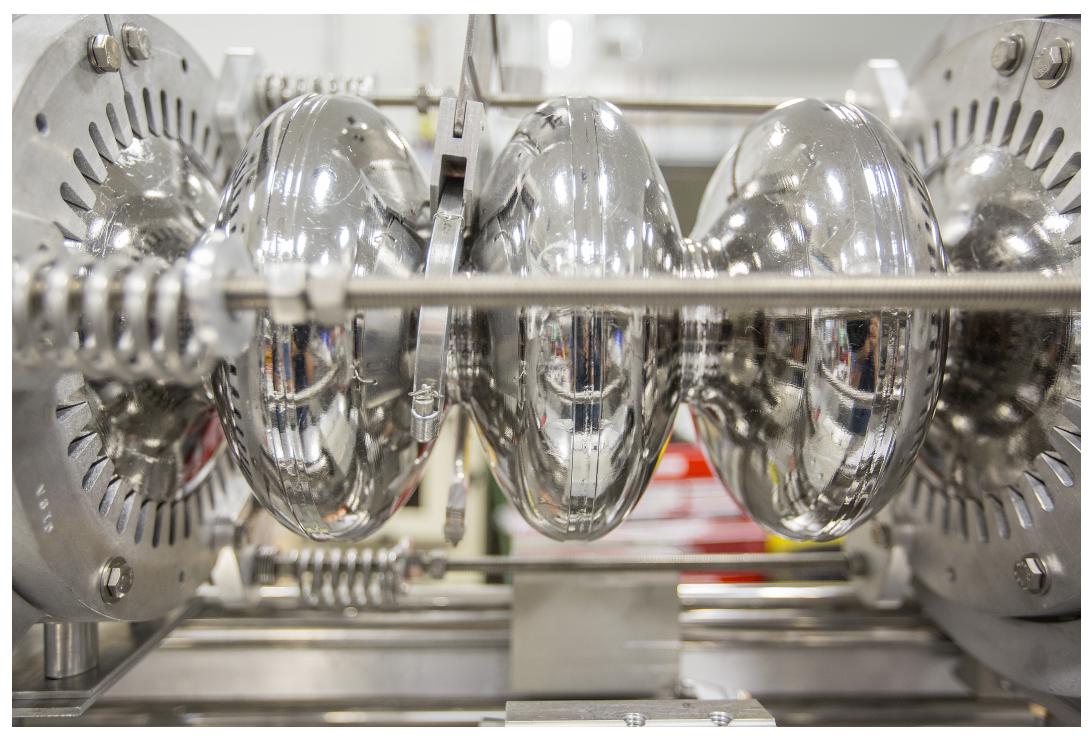

Fig. 3.3. Photo of a Niobium Cavity.

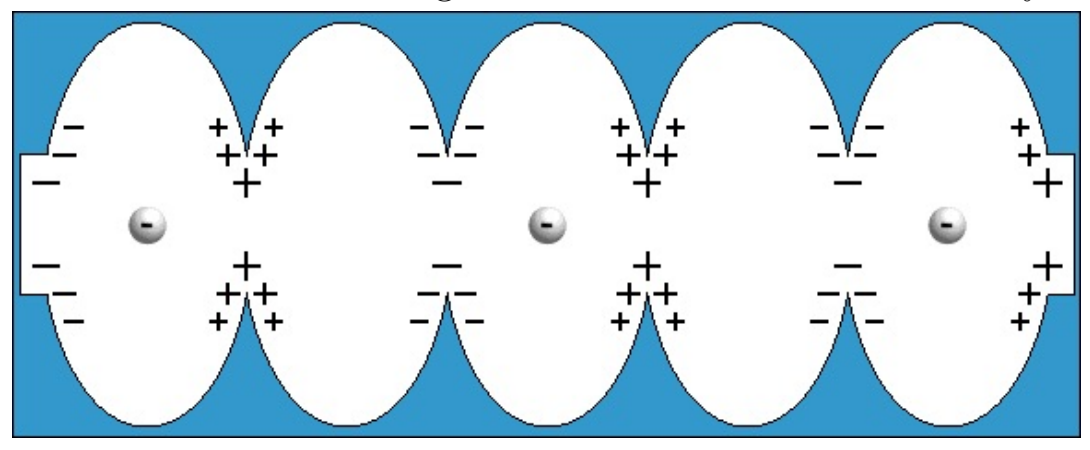

Fig. 3.4. Charge distribution in the cavity. 
increased by $0.6 \mathrm{GeV}$. LINACS are joined by nine recirculation arcs. Depending on the energy of the beam, 16 or 32 magnetic dipoles are used to bend the electrons $180^{\circ}$ (see a picture of the arcs in figure 3.5) around the racetrack. When the electron beam has reached the desired energy, it is finally extracted for each of the experimental halls, individually, using a system of five warm sub-harmonic RF separator cavities operating at $499 \mathrm{MHz}$. For this thesis, we will focus on experimental setup in Hall B. In particular, we will describe the subsystem within the CLAS detector (see section 3.3 below) used for the g13b run period specified in section 3.4 .

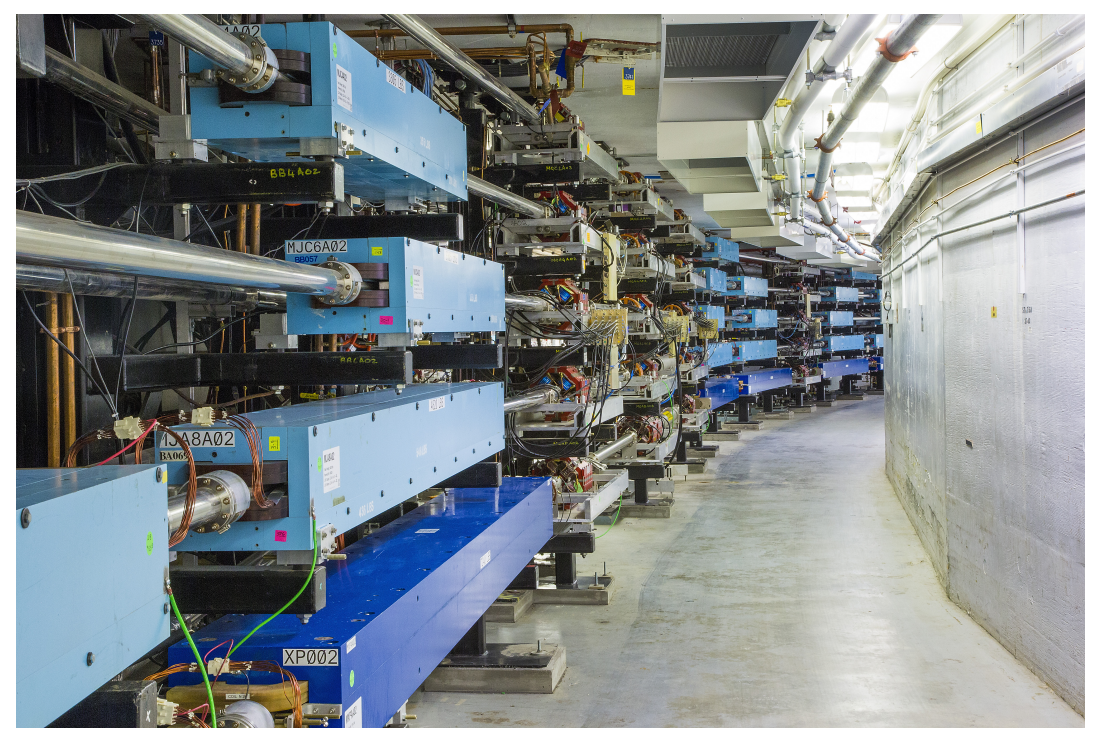

Fig. 3.5. Picture of the magnets in the arcs at Jefferson Lab.

\subsection{Coherent Bremsstrahlung Facility}

\subsubsection{Coherent Bremsstrahlung}

The bremsstrahlung technique is used for photon-induced experiments at Hall

$\mathrm{B}$ in JLab. It consists of the high energy electron beam interacting with the 
magnetic field of a radiator's nuclei. This interaction causes a deceleration of the electron, leading to the emission of a photon. A small amount of the momentum (called recoil momentum) will be transferred to the nucleus to ensure conservation of energy and momentum of the total system. The spectrum of the emerging electrons is not monoenergetic, but it is proportional to $1 / E_{\gamma}$, where $E_{\gamma}$ is the energy of the photon. Three main possibilities are currently used for photon beam experiments: unpolarized, circularly polarized, and linearly polarized. Each one of these possibilities allows us to extract different physics observables. Depending on the type of radiator and whether or not the electron beam is polarized, we can obtain one of these possibilities. In particular, the g13b experiment uses what is called Coherent Bremsstrahlung (CB) to produce linearly polarized photons.

When the direction of incidence of an unpolarized electron traveling through a crystal is close to a direction of major crystal symmetry, the transition amplitudes from the bremsstrahlung may interfere constructively (the amplitudes add up with definite phases) $[23,24]$. More specifically, two conditions imposed in the recoiled momentum have to be satisfied to obtain $\mathrm{CB}$ :

1. "pancake condition": which imposes the longitudinal $\left(q_{l}\right)$ and transverse $\left(q_{t}\right)$ components of the recoil momentum to be in a pancake-shaped region. These limits come from both kinematic restrictions and the fact that the bremsstrahlung cross section decreases rapidly with increasing recoil momentum. A good approximation of the pancake restrictions is presented in the following relations, 


$$
\begin{aligned}
\delta & \leq q_{l} \leq 2 \delta, \\
0 & \leq q_{t} \leq 2 x, \\
\text { with } \delta(x) \equiv q_{l}^{\min }= & \frac{1}{2 E_{0}} \frac{x}{1-x},
\end{aligned}
$$

where $x=\frac{E_{\gamma}}{E_{0}}$ is the fractional energy of the photon, $E_{0}$ is the energy of the electron beam.

2. The Laue condition, where the recoil momentum direction has to coincide with the direction of a reciprocal lattice vector.

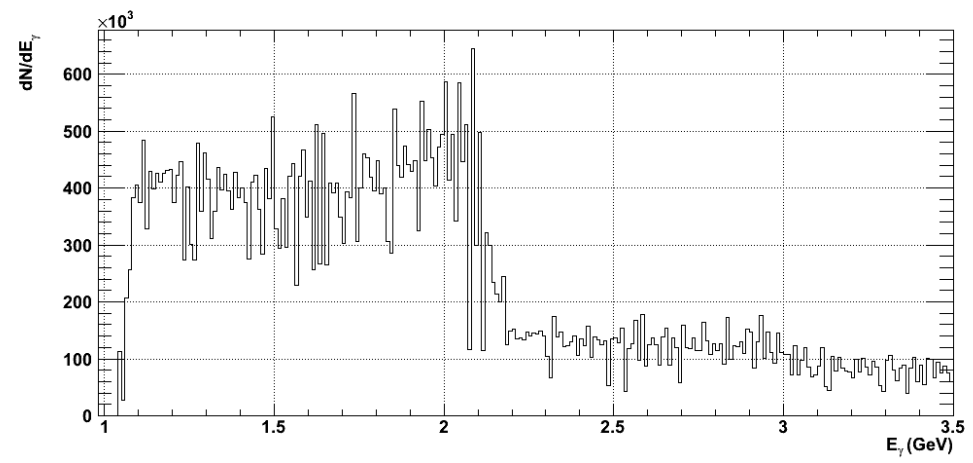

Fig. 3.6. Energy spectrum of linearly polarized photons, showing the coherent enhancement over the incoherent background. Data were taken during the g13b experiment, for a coherent edge of $2.1 \mathrm{GeV}$.

As a result, the total spectrum consists of a coherent enhancement on top of the incoherent, $1 / E_{\gamma}$ spectrum (figure 3.6). The relative intensity plot (or enhancement) shows the ratio of the total coherent spectrum produced using the diamond radiator, and the incoherent energy spectrum from the carbon radiator (see figure 3.7). The most prominent peak corresponds to photons with highest linear polarization and is referred to as the primary "coherent peak". The lead- 
ing edge of the coherent peak is called the "coherent edge". For fixed electron energy, the position of the coherent peak is precisely adjusted by aligning the diamond radiator axis to the electron beam. In the next section, the high-precision goniometer used for this alignment is described.

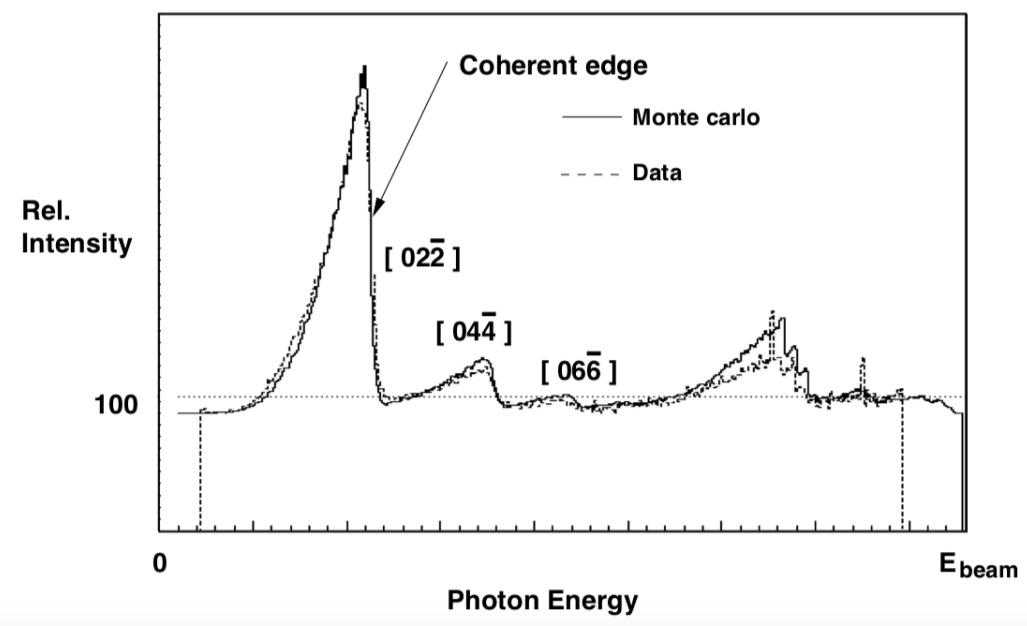

Fig. 3.7. Typical enhancement plot. Taken from [25]. Copyright 2018, with permission from Elsevier.

\subsubsection{Diamond Radiator and Goniometer}

In the g13b experimental period, the radiator was a $50-\mu \mathrm{m}$ diamond positioned $22 \mathrm{~m}$ upstream of the tagger. The experiment also collected a few unpolarized photons using an amorphous radiator. All these radiators were mounted in a target ladder located in the center of a high-precision goniometer (see appendix A from [26]). This apparatus was designed to accurately align the diamond, allowing control over five independent degrees of freedom: three rotational and two translational (See figure 3.8). The goniometer had an angular accuracy between $0.7-1.3 \mu \mathrm{rad}$ and a translational accuracy between $25-180 \mu \mathrm{m}$. In the g13b experiment, the electron beams were varied between $3.3-5.5 \mathrm{GeV}$ producing six 
coherent peak positions ranging from 1.1 to $2.3 \mathrm{GeV}$. The width of the coherent peak was typically $200 \mathrm{MeV}$ with a maximum polarization in the primary coherent peak of $\sim 90 \%$. For each subset of data with a different coherent peak position, the direction of the electric field of the photon was set to two orthogonal orientations: parallel (PARA) and perpendicular (PERP) relative to the floor of Hall B.

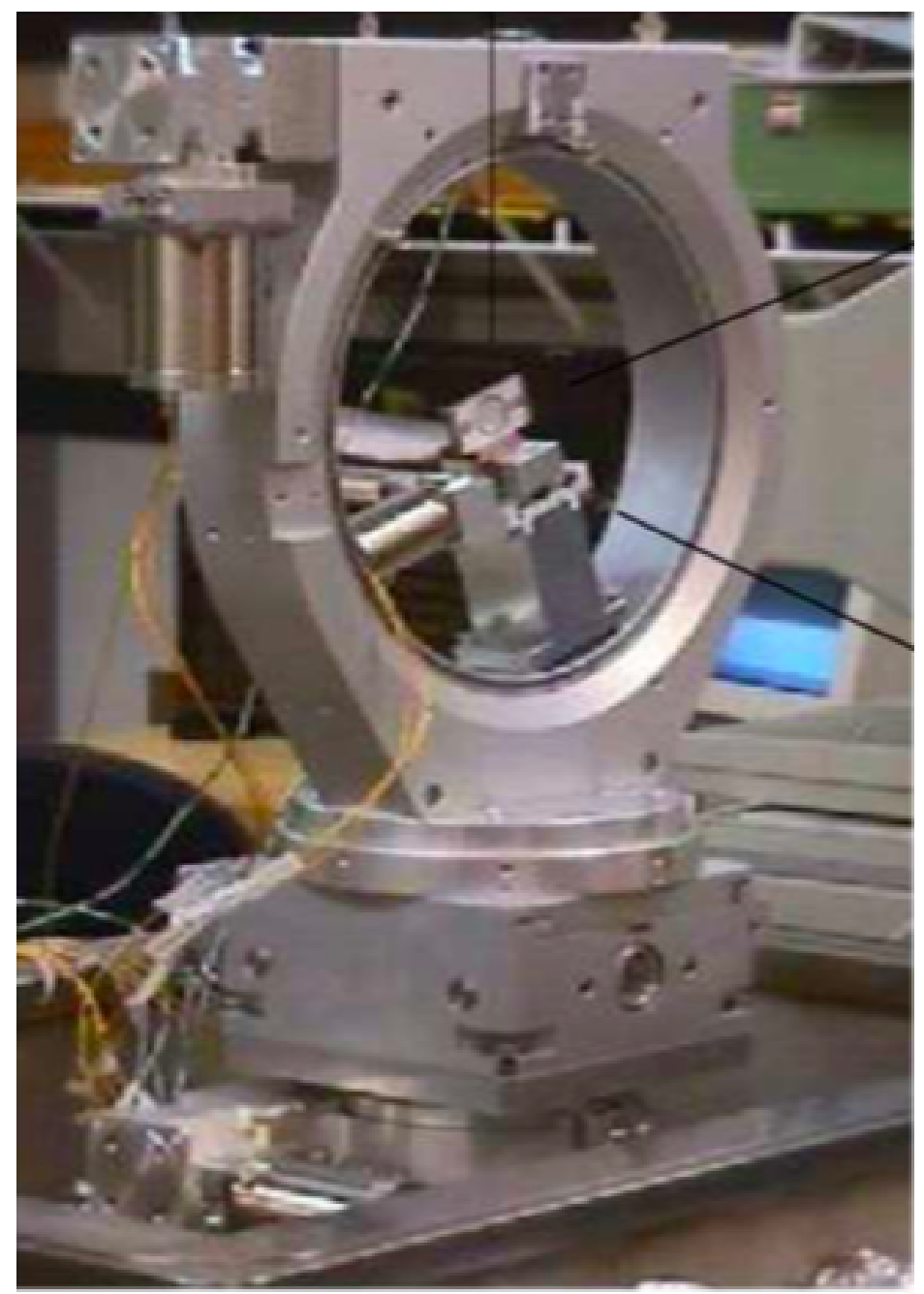

Fig. 3.8. Goniometer 


\subsubsection{Active Collimator}

While the angular distribution of incoherent bremsstrahlung photons is independent of the photon energy, the angle of the polarized photons is directly correlated to the relative photon energy, $x$, and decreases with increasing photon energy. The expression for the reduced angle, $\bar{\theta}$, in units of characterization angle is

$$
\bar{\theta}=\frac{1-x}{x} \frac{x_{d}}{1-x_{d}}-1
$$

where $x_{d}=\frac{2 E_{\mathrm{in}} q_{l}}{m_{e}^{2} \mathrm{c}^{3}+2 E_{\mathrm{in}} q_{l}}$ is the fractional energy. The characteristic angle $\theta^{*}$ is given by

$$
\theta^{*}=\frac{m_{\mathrm{e}} c^{2}}{E_{\text {in }}}
$$

where $E_{\text {in }}$ is the initial energy of the electron. An active collimator placed downstream of the photon tagger was used to reduce the incoherent radiation, enhancing the coherent part of the distribution. The photon beam must be collimated with a $2 \mathrm{~mm}$ aperture, for a collimation of half the characteristic angle [27], in order to achieve a beam polarization with an average of $70 \%$. Finally, it is important to note that the degree of linear polarization, which is fundamental to extract certain polarization observables, cannot be directly measured. This value was obtained by using an analytic expression, using the parameters extracted by fitting the enhancement with a model that takes into account all the relevant parameters.

\subsubsection{Photon Tagger}

The photon tagger [28] aims to tag the energy and time of the bremsstrahlung photons while deviating the electrons away from the beamline. The energy of 
the photon $E_{\gamma}$ can be determined from $E_{\gamma}=E_{\text {in }}-E_{\text {out }}-E_{\text {rec }}$, where the $E_{\text {in }}$ is the energy of the incident electron, $E_{\text {out }}$ is the energy of the outgoing electron, and $E_{\text {rec }}$ is the recoil energy ${ }^{1}$. Since $E_{\text {in }}$ is a known quantity determined by the accelerator and $E_{\text {rec }}$ is negligible, the only unknown energy to calculate $E_{\gamma}$ is the scattered electron energy. By tagging the time and energy of the outgoing electron using momentum analyzing dipole magnet and hodoscope, we can identify the energy of the photon on an event-by-event basis .

For the energies used in the g13b experiment, the characteristic angles of both electrons and photons are smaller than $0.15 \mathrm{mrad}$, which means that both the electron and photon are approximately still moving along the beamline. The beam at this point is composed of scattered and unscattered electrons, and the bremsstrahlung photons. The electrons are deflected by a magnetic field that is tuned to deflect the unscattered electrons into a shielded beam dump located in the floor of the hall. On the other hand, the scattered electrons deviate to a hodoscope with the first plane of E-counters and the second plane of T-counters as shown in figure 3.9. Knowing the magnetic field and the radius of curvature of the electron allows us to calculate its momentum and energy.

A precise and accurate determination of the position of the intersection of the electron trajectory and a plane (E-counter plane) is fundamental in order to extract the radius curvature. The hit position in the hodoscope is determined by 384 scintillator paddles, each $20 \mathrm{~cm}$ long, $4 \mathrm{~mm}$ thick and $6-14 \mathrm{~mm}$ wide. The variation in the width takes into account the variation of the momentum of the electrons, providing a broad tagging range between $0.20-0.95 E_{\text {in }}$. The paddles overlap by one third of their widths on each side, creating 767 channels with a final resolution of $\sim 10^{-3} E_{\text {in }}$.

\footnotetext{
${ }^{1}$ The recoil energy is the energy transferred to the crystal radiator.
} 


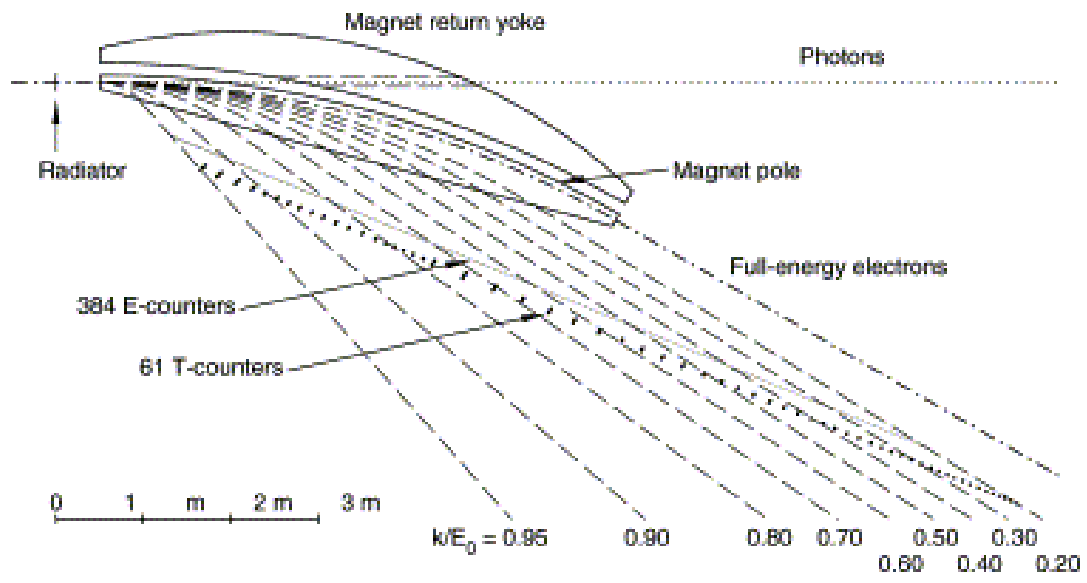

Fig. 3.9. Photon tagger.

Similarly, the T-counter plane contains 61 scintillator paddles, each $2 \mathrm{~cm}$ thick. The T-counter plane is positioned parallel to the E-counter plane, and the former sits $20 \mathrm{~cm}$ behind the latter. To maintain a uniform counting rate, the widths of the scintillators vary to compensate for the $1 / E_{\gamma}$ distribution of the incoherent bremsstrahlung. The T-counters slightly overlap, creating a total of 121 channels with a time resolution of 110 ps [28]. Furthermore, the T-counters use two PMTs at either end for better timing resolution.

\subsection{CLAS Detector}

The CEBAF Large Acceptance Spectrometer (CLAS) is devoted to studying nuclear and hadronic reactions produced from both electrons and photons by detecting the final-state particles. For charged particle detection, it provides coverage of almost the full $4 \pi$ solid angle. CLAS is located in Hall B at Jefferson Lab, and it is composed of several detector subsystems within a toroidal geometry. The torus consisted of six superconducting coils that produced a nonhomogeneous magnetic field that allowed it to bend charged particles. Each one of the super- 


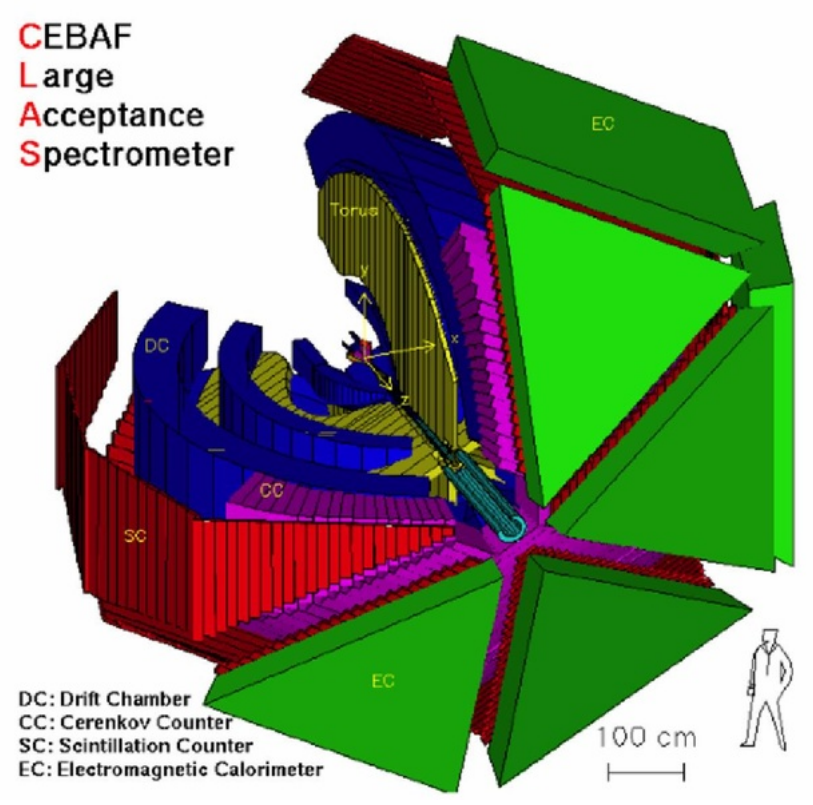

Fig. 3.10. Experimental Layout of the CLAS detector before the $12 \mathrm{GeV}$ upgrade. Picture taken from [22].

conducting coils divided CLAS in six independent sectors [22]. The real photon experiments used four major subsystems: STart counter (ST), Drift Chambers (DC), Time of Flight (TOF) scintillators and Electromagnetic Calorimeter (EC). The Cherenkov counters (CC) are not used for photon-induced experiments since CC's primary goal is to differentiate between electrons and pions. The sections below present a description of the torus magnet and the detectors used in this thesis.

\subsubsection{Torus Magnet}

The torus magnet is composed of six superconducting coils located around the beamline and shaped in a toroidal geometry (figure 3.12). Each coil separates the detector naturally into six independent spectrometers referred to as sectors. The symmetry of the magnet provides large coverage while preserving a central 
field free region [22]. As a result, the largest component of the magnetic field lies in the $\phi$ direction except for small deviations at the regions close to the coils (figure 3.11).

The magnet's dimensions are $5 \mathrm{~m}$ in diameter and $5 \mathrm{~m}$ in length. Each coil consists of four layers of 54 turns of aluminum-stabilized $\mathrm{NbTi} / \mathrm{Cu}$ conductor. The coils are cooled to $4.5 \mathrm{~K}$ by a tubing system running super-critical helium and are highly isolated to ensure and maintain superconducting conditions.

As described in the section 3.4, the magnetic field had a negative polarity at a torus current of $-1500 \mathrm{~A}$. This is referred to as out-bending data meaning that the magnetic field bent the negatively-charged particles away from the beamline (and thus positive ones toward the beamline).

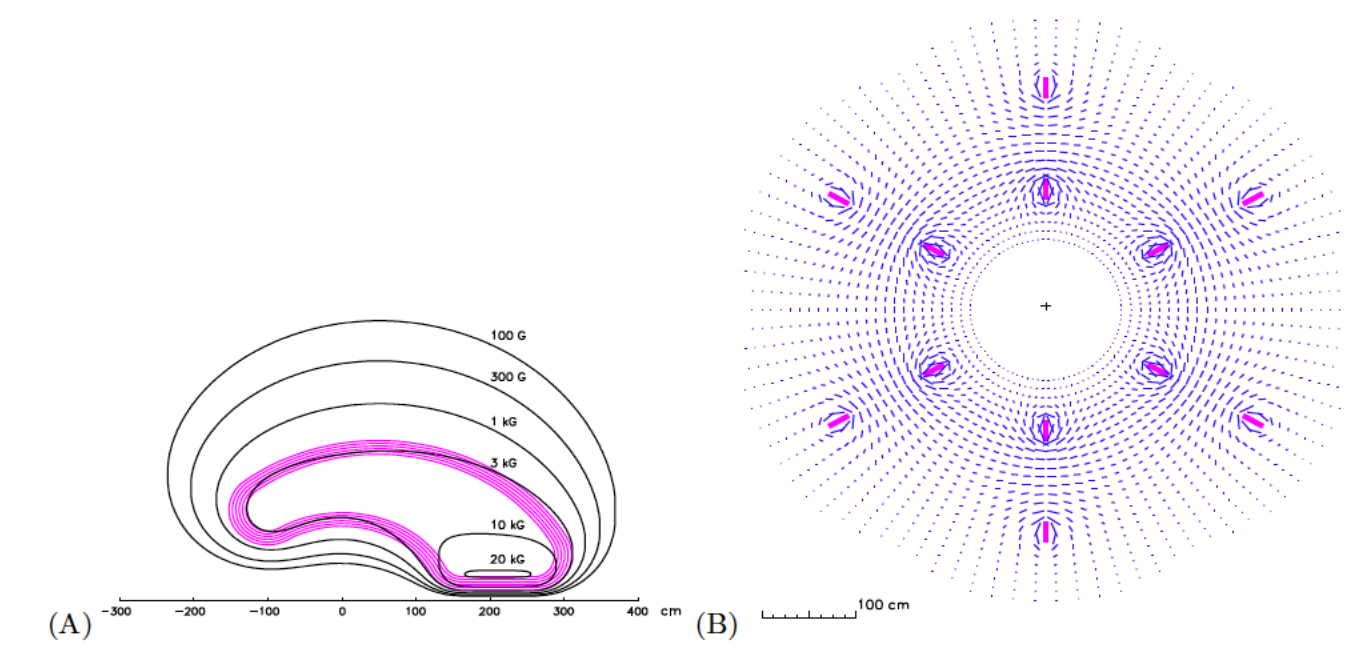

Fig. 3.11. a. Magnetic Field contour plot in the middle plane between coils. b. Magnetic field perpendicular to the beamline and centered in the target. The six coils can be seen in the intersection with the plane. Image taken from [22] with permission. 


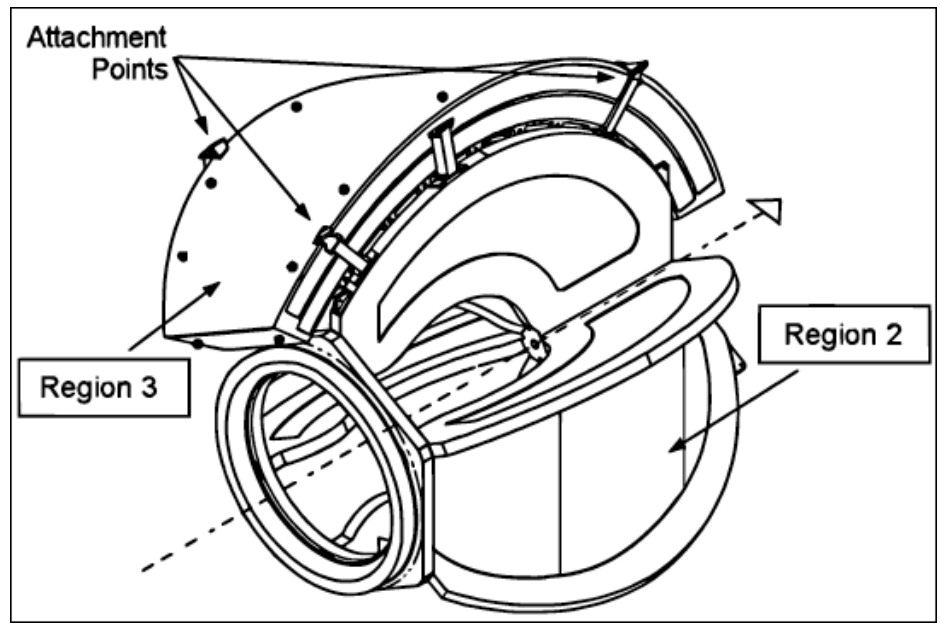

Fig. 3.12. Schematic view of the torus cryostat. Image taken from [22] with permission.

\subsubsection{Start Counter}

The start counter is used only in real photon beam experiments. It is placed around the target cell; therefore, it provides a fast input for the trigger. It also records the initial time of every event generated in the target that set a trigger. The determination of the start time, together with the time from the Time Of Flight (TOF) subsystem, is used to calculate the time of flight of the outgoing particles. The start counter is composed of 24 scintillator paddles, 4 for each sector, arranged in a hexagonal configuration around the target cell. Each paddle is a single continuous piece of EJ-200 scintillator, with a 502-mm straight section referred to as the "leg" and a tapered end called the "nose" [29]. When a charged particle passes through a scintillator paddle, radiated photons are induced from ionization, and they are collected by photomultiplier tubes attached at the end of each paddle.

The time resolution for the "leg" section is $292 \pm 1$ ps while it is $324 \pm 2$ ps for the "nose" [29]. This resolution is sufficient to be able to identify the photon 


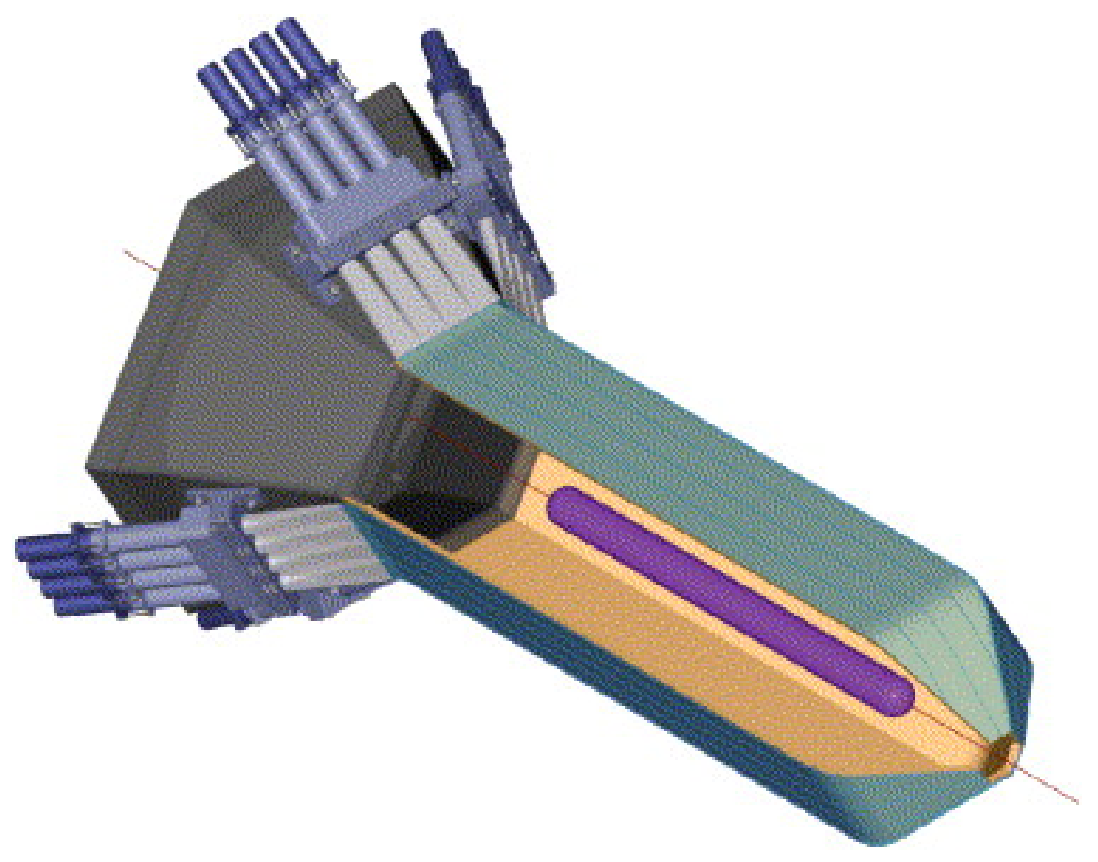

Fig. 3.13. Schematic 3D view of the start counter. The purple volume represents the target cell. Image taken from [29] with Elsevier permission.

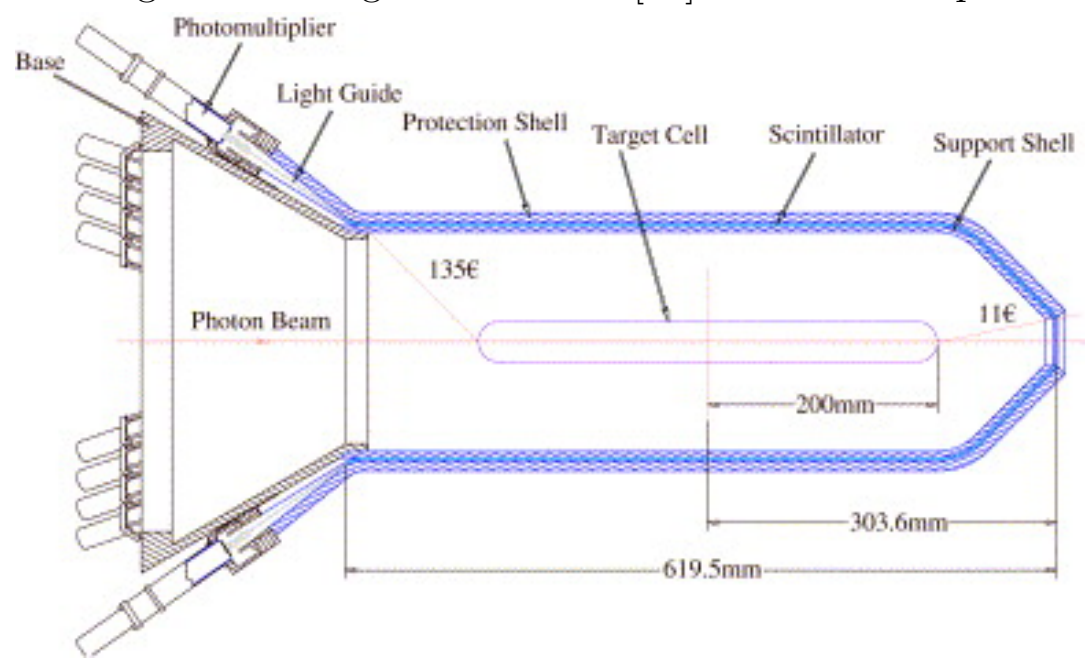

Fig. 3.14. Schematic cross section of the start counter subsystem. Image taken from [29] with Elsevier permission. 
bunch that initiated a reaction.

\subsubsection{Drift Chambers}

The Drift Chambers (DC) form the charged particle tracking system [30]. This subsystem is composed of 18 chambers, partitioned into three regions (R1, R2, R3). R1 is located before the magnetic field, R2 in the magnetic field, and R3 after the magnetic field in each sector (see figure 3.15). Each chamber is composed of two superlayers of 6 wire-layers each and is filled with a mixture of 90\% Argon and 10\% $\mathrm{CO}_{2}$ gas. Six field wires surround a sense wire creating a hexagonal cell. The field wires are set to a positive high voltage, while the sense wire is maintained at a negative high voltage, producing an electric field. In each region, the wires of one of the two superlayers are oriented axially relative to the magnetic field direction, while the wires from the other superlayer are oriented at a $6^{\circ}$ stereo angle. Charged particles crossing the chambers ionize the gas (in this case Argon). The electrons then drift, due to the electric field, toward the anode, while positive ions will drift to the cathode. Near the anode, the electric field is big enough for the electron to knock an electron from the molecules of the gas. This secondary electron can also ionize molecules in its way to the anode, and the repeated process forms an avalanche. $\mathrm{CO}_{2}$ serves as a quencher. When the electrons reach the sense wire, the drift time can be measured, and hence the distance of closest approach. This information together with the magnetic field information is used to reconstruct the trajectory of the particles inside the drift

chambers. A resolution of $\frac{\delta p}{p} \leq 0.5 \%$ was obtained for a nominal value of electron momentum of $1 \mathrm{GeV} / \mathrm{c}$. 


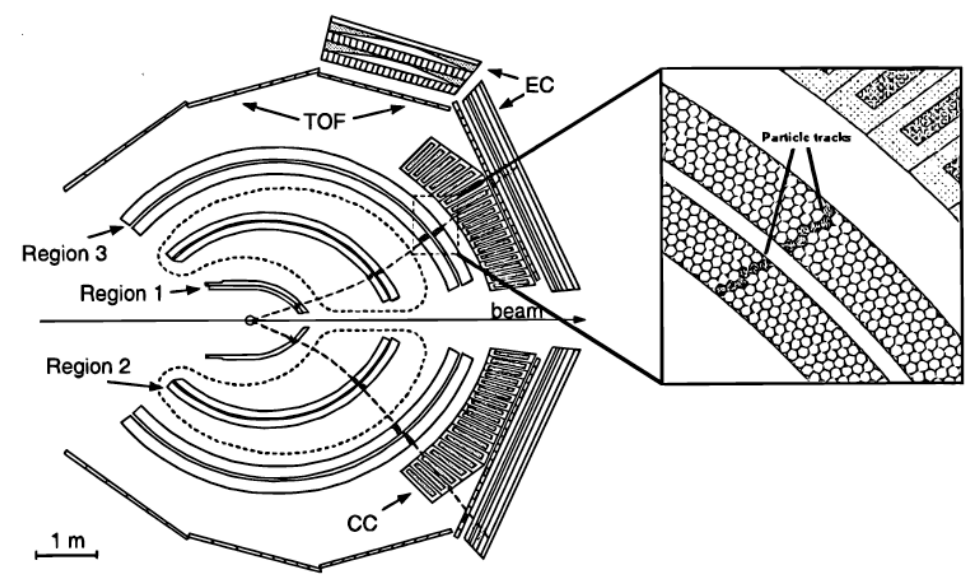

Fig. 3.15. Schematic 2D view of the CLAS detector cut at the beamline high. It shows a representation of two charged particles being detected. The dotted lines represent the projection on this plane of the torus coils position. Enlargement in the box exemplifies the hit pattern in the two superlayers of the region 3 of the drift chambers. The hexagonal cells are discussed in more detail in the text. Image taken from [30] with Elsevier permission.

\subsubsection{Time of Flight}

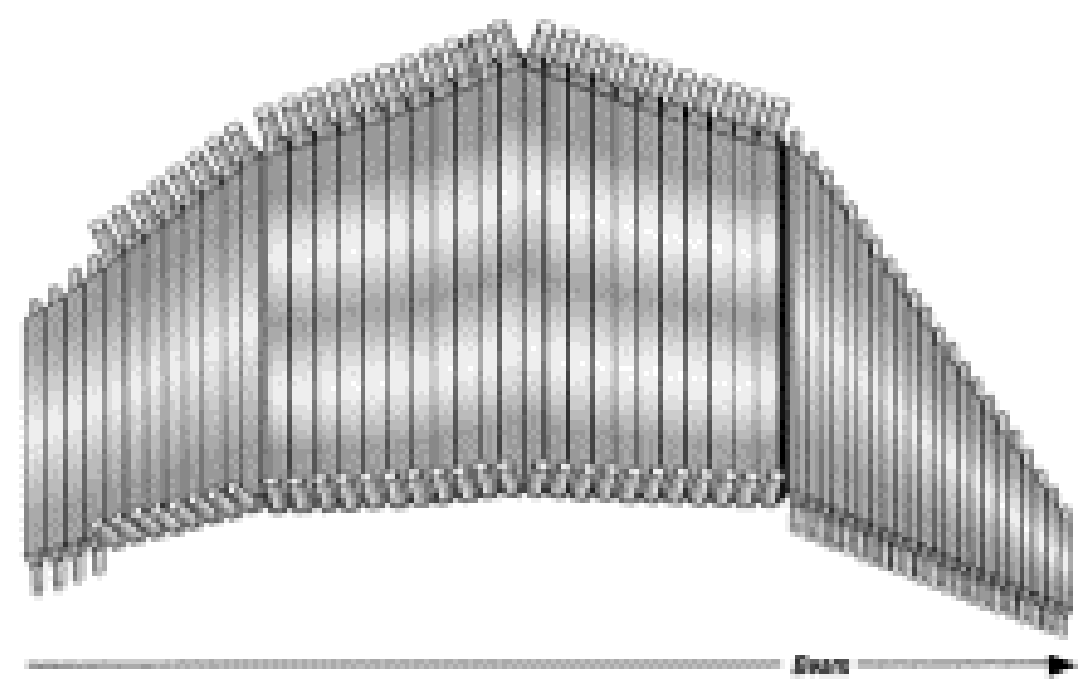

Fig. 3.16. TOF scintillator shell from one sector. Picture taken from [31] with Elsevier permission.

The Time of Flight (TOF) [31] is an arrangement of 336 plastic scintillator counters located 3.5 to 5 meters from the center of CLAS. The TOF subsystem 
covers a polar angle of $8^{\circ} \leq \theta \leq 142^{\circ}$. In each sector, the scintillator wall consisted of four panels of 57 bars of different lengths and widths (see figure 3.16). The TOF provides information of the time it takes a particle to travel from the reaction vertex in the target, through the DC, and then hit the TOF scintillator paddles. Thus, the time of flight is calculated as the difference between the time measured in the TOF and the time in the vertex. This information, in combination with the path length provided by the DC, is used to calculate the relative velocity of the particle which, in turn, is used for charged particle identification. The scintillator fluoresces as it is excited by ionizing radiation coming from a charged particle passing through the material. A good portion of these photons are reflected through the light guides into the photomultiplier tubes (PMTs) at each end. The overall time resolution is 150 ps.

\subsubsection{Electromagnetic Calorimeter}

The Electromagnetic sampling Calorimeter (EC) [32] was designed to accomplish three main functions: to improve the efficiency of differentiation between electrons and pions for energies above $0.5 \mathrm{GeV}$, to allow studies in the mass reconstruction of neutral particles that decay in two or three photons, and to detect neutrons. This subsystem was particularly relevant in this work because, to reconstruct the $\pi^{0}$ meson, coming from the $\omega$ meson decay $\left(\omega \rightarrow \pi^{+} \pi^{-} \pi^{0}\right)$, we have to detect the two photons obtained in the decay $\pi^{0} \rightarrow \gamma \gamma$.

The EC is composed of alternating layers of the bc412 scintillator and lead $(Z=82)$ sheets; each sector has 39 layers with equilateral triangle shape (see figure 3.17). Each scintillator layer has 36 strips aligned with one of the sides, and each consecutive layer is rotated $120^{\circ}$. Three consecutive layers form a group of $\mathrm{U}$, 
$\mathrm{V}$, and $\mathrm{W}$ views, each containing 13 layers, providing information of the energy deposited and the position. Each view is further subdivided into two regions: inner (5 layers) and outer (8 layers).

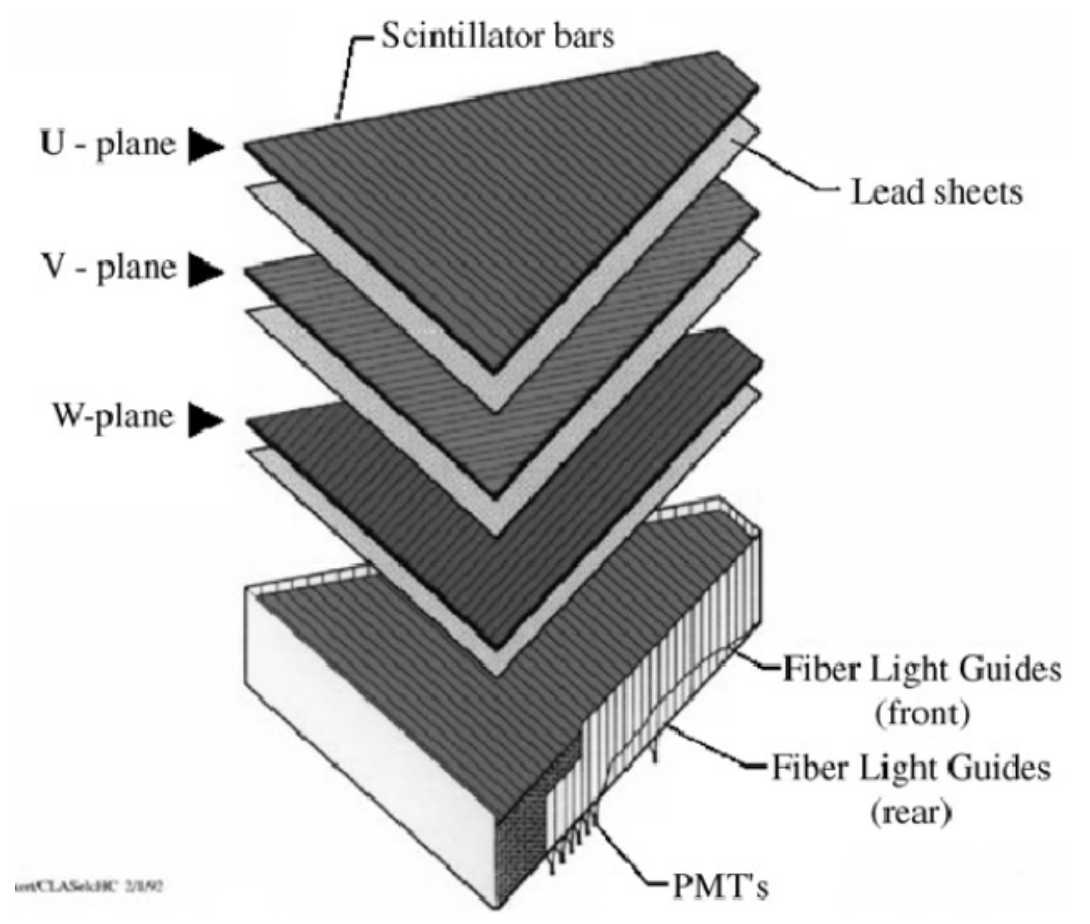

Fig. 3.17. Deconstructed view of one of the electromagnetic modules. Image taken from [32] with Elsevier permission.

When a particle hits a lead layer, it creates a shower which produces photons in the scintillators. The light is collected by the PMT and sent to the ADC (analog to digital converter) and to the TDC (time to digital converter) boards. The sampling fraction, which is the fraction of energy deposited in the active part of the calorimeter, is estimated to be 0.273 . The energy resolution is $\frac{\Delta E}{E}=$ $0.003+\frac{0.093}{\sqrt{E(\mathrm{GeV})}}$ and the time resolution is about 600 ps for neutral particles, which is $\sim 10 \%$ for $1 \mathrm{GeV}$. 


\subsection{The g13b Experiment}

The CLAS g13 experiment was performed between October 2006 and June 2007. The g13 running period had both linear (g13b) and circular (g13a) polarized photon beams incident on a $40 \mathrm{~cm}$ liquid deuterium target [33]. The target is described in the section 3.4.1. In this analysis, we will study the g13b experiment. The linearly polarized photons were obtained via the bremsstrahlung process with an unpolarized electron beam incident on a $50-\mu \mathrm{m}$ diamond radiator. The orientation of the diamond radiator (located in a goniometer) made it possible to define the photon polarization plane and the energy of the coherent peak. The range of energies of the incoming photons ranged from 1.1 to $2.3 \mathrm{GeV}$ with the coherent peak set every $200 \mathrm{MeV}$, for a total of 6 different settings. This was obtained by changing the diamond orientation, and the relative energy of the energy of the photon.

The collimation enhanced the polarization because the angle of emission of the coherent bremsstrahlung is smaller than the one for the unpolarized background. The polarization of the radiated photons in the coherent peak was estimated to be between $70 \%$ and $90 \%$. The electron bremsstrahlung technique is described in detail in section 3.2. The beam current was $10 \mathrm{nA}$. The torus magnet was set to bend negatively charged particles outward from the beamline, with a current of -1500 A. A single sector trigger was used producing approximately 30 billion events recorded on tape during the whole running period.

\subsubsection{Target Cell}

The Kapton target cell had a slightly conical shape (see figure 3.18) with a thin aluminum window at the front. The target cell was designed to hold either liquid 
hydrogen or liquid deuterium using a cryogenic system.

The target was 40-cm long and had a maximum diameter of $4 \mathrm{~cm}$. It was located $20 \mathrm{~cm}$ upstream from the center of CLAS to maximize acceptance. The length of the target ensured high statistics while maintaining a good rate of photons in the tagger. The width of the cell provided coverage for the $\sim 1 \mathrm{~cm}$ spread of the photon beam and minimal beam misalignment. Most of the runs for the g13 run period used a liquid-deuterium target which has a density of $0.1625 \mathrm{~g} / \mathrm{cm}^{2}$.

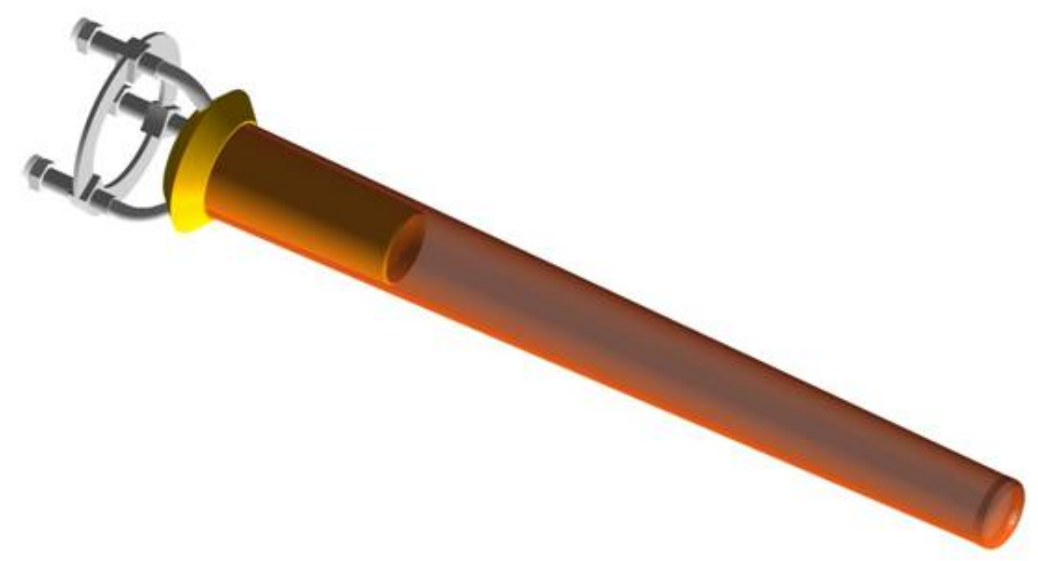

Fig. 3.18. Liquid-deuterium target cell. Picture taken from [34].

\subsubsection{Trigger Conditions}

Not all the information registered by each subsystem corresponds to an event of interest. It is fundamental to establish an optimal trigger system to select events of interest while minimizing the dead time. In general, CLAS has a twolevel hierarchical system [22]. The level 1 trigger allows the use of all available prompt information coming from the PMT channels, while the level 2 trigger 
uses the slower track information from the drift chambers to determine whether to write an event to the disk. The g13 experiment only used a level 1 trigger. In particular, for the g13a run period, a two-sector coincidence between the ST and the TOF was used. On the other hand, the g13b experimental period required only one sector coincidence between the ST and the TOF.

\subsubsection{DAQ}

After the trigger conditions are satisfied, the data acquisition (DAQ) system records the information onto magnetic tape. For the g13 period, the physics events were recorded at a rate of around $10 \mathrm{kHz}$ with a dead time of approximately $15 \%$. In total, the number of events recorded for the g13a run period was $\sim 20$ billion, while for g13b the number was $\sim 30$ billion. The average size for each event is around $2.5 \mathrm{kB}$, making a total of $\sim 130 \mathrm{~TB}$ dataset. These raw data were recorded in Bank Object System (BOS) format, and each bank contains the information of the particular subsystem position and electric signals ready for

off-line analysis. To extract physical quantities from these data, a complex and multi-step process has to be performed. In the next chapter, we will discuss the process of reconstructing a physical event. 


\section{Chapter 4}

\section{Data Analysis: Event \\ Reconstruction}

\subsection{Overview}

The data coming from CLAS undergo a multistep process before being used in a physics analysis. The "raw" data coming from the different subsystems first have to be "cooked"; this means we have to calibrate each one of the subsystems. The calibration is applied only for a selected number of runs and by iteration until the data reach a stable value after which the calibration constants are frozen. After this, data are available for reconstruction of physical quantities that will be used to identify different channels. For this particular analysis, we have $\vec{\gamma} d \rightarrow p \omega(n)$, where the particle in the parenthesis denotes the "spectator". In the spectator model, it is assumed that the photon interacts only with one of the nucleons, in this case the proton, while the neutron acts only as a spectator. It is in general assumed the impulse approximation, implying that during the collision there is no three-momentum transferred to the spectator. As a consequence, the momentum 
of the neutron in the final state corresponds to the Fermi momentum it had inside the deuteron at the time of the interaction. Therefore, the neutron spectator will have a momentum lower than $\sim 200 \mathrm{MeV}$, and it will not be possible to detect it with the EC subsystem. Because of this, we have to identify all final state particles from the decay.

This chapter explains in detail the method that we used to exclusively identify

$$
\begin{aligned}
& \gamma d \rightarrow \omega \quad p(n), \\
& \omega \rightarrow \pi^{+} \pi^{-} \pi^{0}, \\
& \text { and } \quad \pi^{0} \rightarrow \gamma \gamma
\end{aligned}
$$

The branching ratio for the charged decay channel of the $\omega \rightarrow \pi^{+} \pi^{-} \pi^{0}$ is $89.2 \%$ and for the $\pi^{0} \rightarrow \gamma \gamma$ decay is $98.8 \%$.

This chapter explains in detail the method that we used to identify the finalstate particles of the decay described in equation (4.1) and the reconstruction of the $\omega$ meson. The particles to be detected are five: the recoil proton, $\pi^{+}, \pi^{-}$and two photons from the $\pi^{0}$ decay.

\section{2 "Cooking" and Detector Calibration}

The information collected by the CLAS detector subsystems is transferred on an event-by-event basis to a dynamic memory structure called BOS (Bank Operating System) ([35]). The BOS format consists of several banks ${ }^{1}$, at least one per subsystem at this stage. The information included in the so-called "raw" BOS files consisted of channel ID and values coming from the Charge to Digital

\footnotetext{
${ }^{1}$ In dynamic memory management field, the term banks refers to structures of similar data. The banks that are the building blocks of the BOS [36].
} 
Converters (QDC) and Time to Digital Converters (TDC).

The "raw" data are then "cooked", which is the process of data reconstruction using the software RECSIS (REConstruction and analySIS package [37]). What this means is that the banks from the original BOS files are now translated into physical quantities like momentum, position, energy, etc., and are stored in new banks in the "cooked" BOS file. It is important to account for differences in the conditions during the whole experimental period by calibrating each CLAS subsystem and the tagger. The complete data range was then divided into subperiods where the experimental conditions were stable. For each subperiod, a "golden" run was chosen for calibration in all the subsystems. For example, figure 4.1 shows the start-counter-offset mean value and the standard deviation $\sigma$ for $\pi^{-}$for an intermediate stage of calibration. The different color lines show the subperiods for g13 and in the bottom the golden runs. The calibration from the start counter will ensure that the mean value $<100$ ps for all the runs while maintaining the standard deviation of $\sim 400 \mathrm{ps}$.

The calibration of all the subsystems is done in a particular order, given that some of the values from individual detectors are used in other calibrations. A group of collaborators performed the calibration for each subsystem: Charles Taylor and Danny Martinez from Idaho State University for the ST and TOF calibration, Edwin Munevar and Nicholas Zachariou for the DC and tagger calibration and Paul Mattione for the EC. Their dedication to this work ensured high-quality data for this analysis. After calibration, the set of "calibration constants" is stored in a centralized MySql database and linked to RECSIS. Data are then cooked and monitored again until the data reach a stable and acceptable condition for physics analysis ${ }^{2}$.

\footnotetext{
${ }^{2}$ The "acceptable conditions" for data vary for each detector. For example, for the start
} 


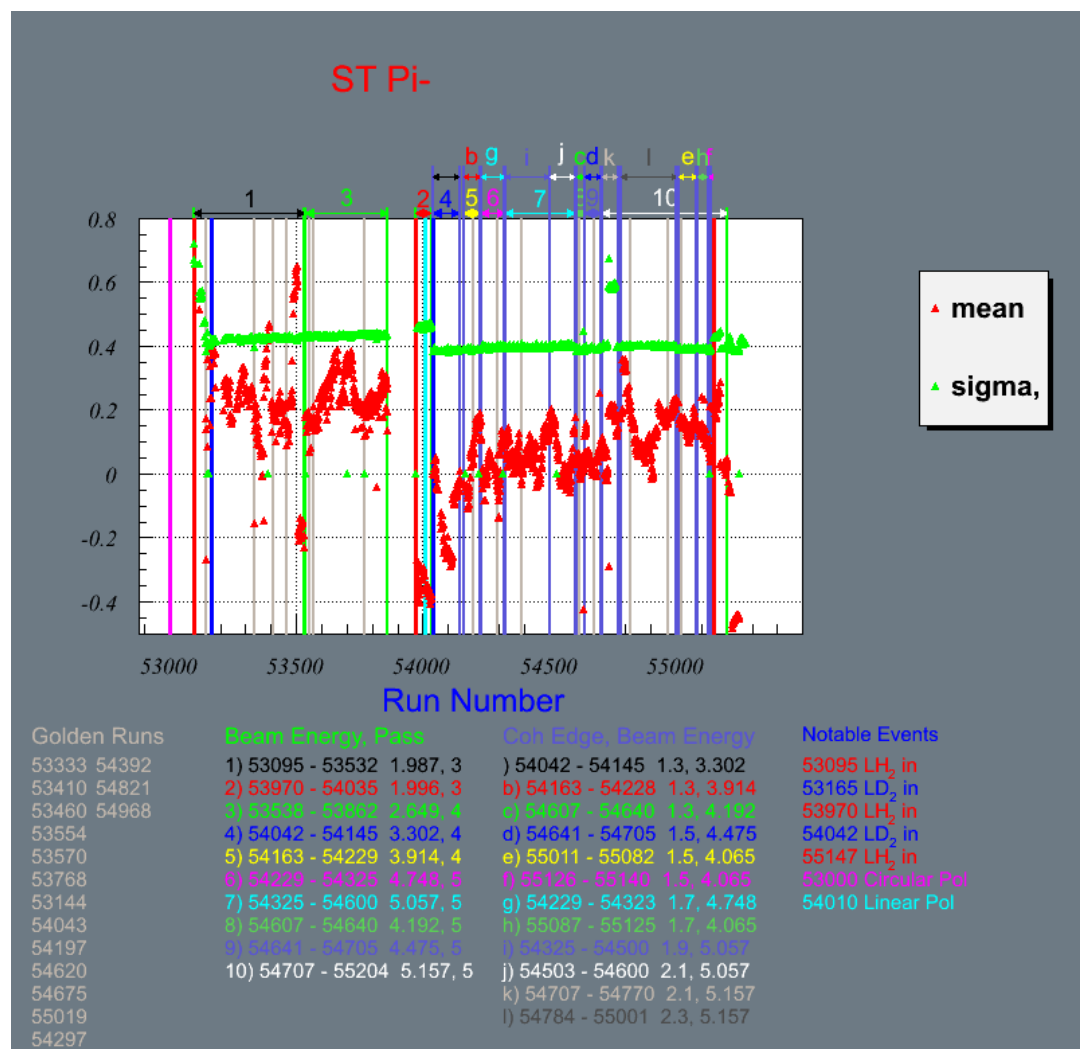

Fig. 4.1. Start Counter offset for $\pi^{-}$particles for an intermediate stage of the Start Counter calibration. The figure shows the subperiod division, and the runs that were chosen to be golden runs. 
The following is a list of banks that were used in the analysis and will be referenced later in this thesis:

- EVNT: this bank has general information about the event after reconstruction.

- MVRT: primary vertex reconstruction calculation by assuming all the final state particles coming from the target.

- EPICS: information about the coherent edge, the radiator, coherent plane.

- TAGR: information from all the incident photons as well as the electron associated with each photon.

- ECPB: Electromagnetic Calorimeter information. This bank was particularly used for the $\pi^{0}$ reconstruction.

For more information on the bank structure of the BOS files, refer to reference [35]. The "cooked" files are now analyzed with ROOT [39], a scientific software framework developed at CERN. ROOT provides big data handling, powerful mathematical and statistical tools, and data visualization.

\subsection{Data Exclusion}

The appropriate selection of good-quality data will translate into a reduction of the systematic uncertainty. During the experimental period, some of the runs were tagged as "junk" from the shift takers, or were taken from different studies counter the mean value of the offset for protons, $\pi^{+}$and $\pi^{-}$were asked to be less than $0.2 \mathrm{~ns}$ while the sigma should be $\sigma<0.5 n s$. Monitoring plots were generated to check if these parameters fulfilled these conditions for almost all the runs. These plots can be seen in reference $[38]$. 
and not for production. A list of "good runs" can be found in the g13 wiki page. This list combines information from the logbook, the g13 wiki, the status obtained from the database, and studies on the EPICs bank.

\subsection{Skimming}

\subsubsection{First SKIM}

Data at the cooking stage were filtered according to the minimum number of tracks. This process is known as "skimming" and allows a faster data analysis as it reduces the file size, by cutting out events that do not correspond to the minimum track requirement. Three topologies were created and the one that was used for this analysis required at least three tracks: one positive, one negative, and one neutral (1pos1neg1neu).

\subsubsection{Second SKIM}

The second skim is a filter that was applied to the skimmed BOS files. This time, the criteria for reducing the data are decay specific, and the skim process retrieves only information that will be later used in this analysis. The resulting root files have only events with the following requirements:

- Two tracks associated with positively charged particles, one track associated with one negatively charged particle and two tracks associated with neutral particles. For all the tracks, a positive status on the EVNT bank was required ${ }^{3}$.

\footnotetext{
${ }^{3}$ According to the dictionary for the BOS banks [35], an out of time particle will be given a 0 status on the EVNT bank.
} 
- The positive track with higher calculated mass is assumed to be a proton. Therefore, the other track is assumed to be $\pi^{+}$. All negative particles are assumed to be $\pi^{-}$, and all neutral particles are assumed to be photons.

- We perform a loose cut on the $\Delta \beta<0.6$ for the positively charged tracks. $\Delta \beta$ will be discussed below.

\subsection{Particle Identification}

After the initial cuts, the good tracks have to be identified as the particles in the final state. In this section, the process to identify a proton, $\pi^{+}, \pi^{-}$and two photons is described.

\subsubsection{Charged Particles}

The relative velocity $\beta \equiv \frac{v}{c}$ in the EVNT bank was calculated with the help of the track length $l$ and the time $t$ values obtained from the TOF subsystem, and the time, $t_{\mathrm{ev}}$, of the event vertex; $\beta_{\text {meas }}=\frac{l}{c\left(t-t_{\mathrm{ev}}\right)}$. The difference between the $\beta_{\text {meas }}$ in the bank and the $\beta_{\text {calc }}$ was calculated for the different tracks,

$$
\begin{aligned}
\Delta \beta & =\beta_{\text {meas }}-\beta_{\text {calc }} \\
\beta_{\text {calc }} & =\frac{p}{\sqrt{p^{2}+m_{\mathrm{PDG}}^{2}}}
\end{aligned}
$$

where $m_{\mathrm{PDG}}$ is the mass of the particle reported by the particle data group [5] and $p$ the momentum of the particle. A $3 \sigma$ momentum dependent cut in the $\Delta \beta$ was performed to identify charged particles. The projections of the $2-\mathrm{D}$ plot

$\Delta \beta$ vs. momentum along the momentum axis were fit with a Gaussian. The 
$\mu \pm 3 \sigma$ profiles were then fit with a polynomial function as described below. The parameters extracted from these fits are then used to define the upper $(\mu+3 \sigma)$ and lower limit $(\mu-3 \sigma)$ for the charged particle identification cuts. For largemomentum and low-momentum bins with poor statistics, an extrapolation of a 0th-order polynomial was performed, taking the value of the closest momentum bin that had good statistics. In figure 4.2 , we can see the $2-\mathrm{D} \Delta \beta$ vs p plot. The red lines represent the final cut to select the proton. Three regions were defined based in the momentum of the track: for $p<0.26 \mathrm{GeV} / \mathrm{c}$ and $p>1.6 \mathrm{GeV} / \mathrm{c}$ were defined with 0th order polynomial, while the central region was fitted with a 6 th order polynomial. In the two plots on the right in figure 4.2, we can see more clearly the fit for the middle region. The bands contaminating the sample can come from different sources: out-of-time particles that correspond to a different electron bunch, misidentified particles, and accidentals. As can be seen in figure 4.2 , most of the background is cut out of the signal, although an additional cut for out of time particles will be performed later in this analysis.

A similar analysis was performed for the identification of pions. The central region $p \in[0.2,1.2] \mathrm{GeV} / \mathrm{c}$ was fit with a 8 th order polynomial. The constants are slightly different for $\pi^{+}$and $\pi^{-}$, but the regions on momentum are the same. The results for $\pi^{-}$can be seen in figure 4.3 and for the $\pi^{+}$in figure 4.4 .

The rest mass $m_{0}$ of the particles can be determined as a function of the two independent measurements, $p$ and $\beta_{\text {meas }}$, as follows:

$$
m_{0}^{2}=\left(\frac{p^{2}}{\beta_{\text {meas }}^{2}}\right)\left(1-\beta_{\text {meas }}^{2}\right) .
$$

The effect of the $\Delta \beta$ cuts on the mass-squared distribution can be seen in figure 4.5 for tracks with positive charge and figure 4.6 for negatively charged particles. 

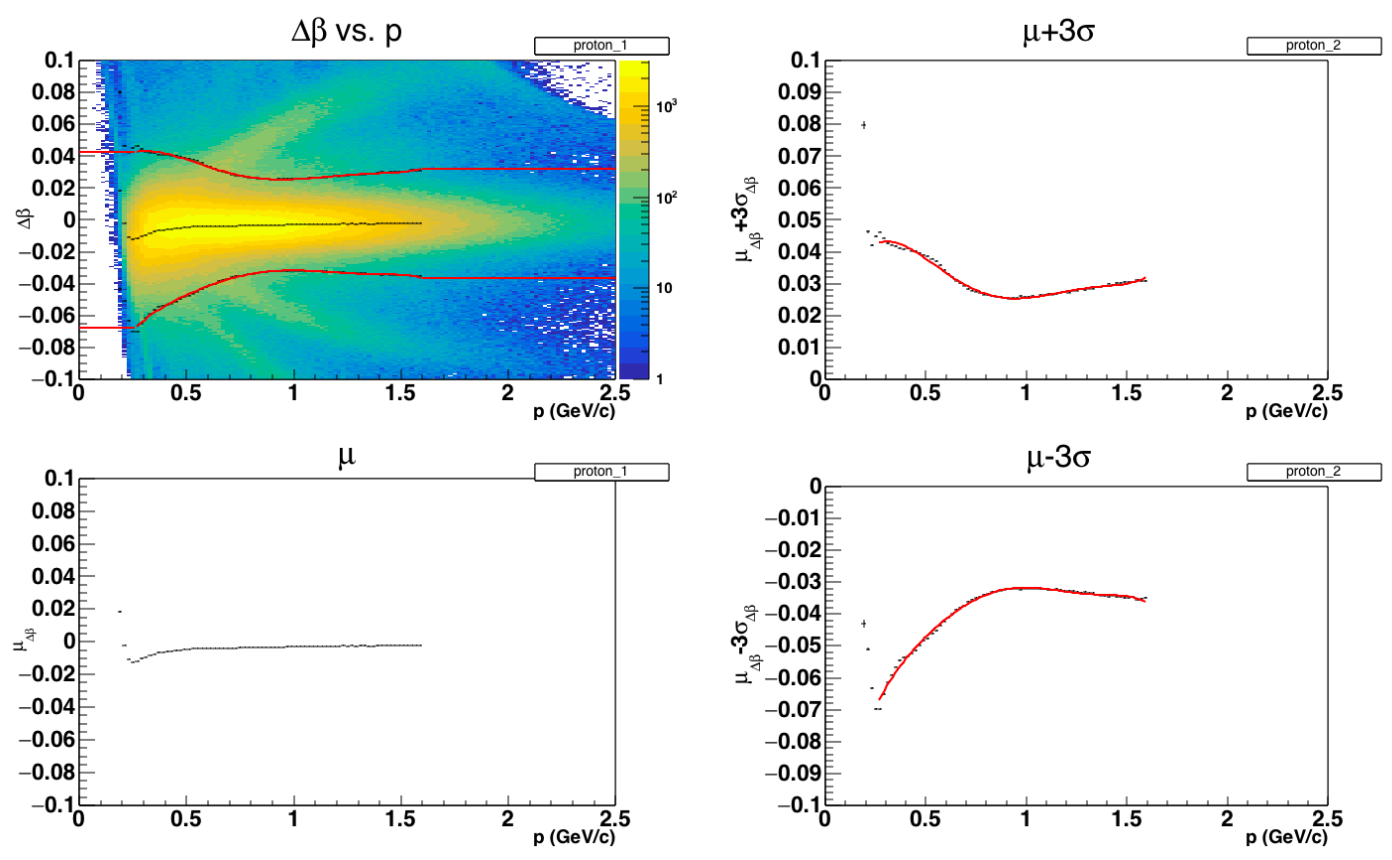

Fig. 4.2. Proton identification. Top left figure is the $\Delta \beta$ vs p plot. The events between the red curves, are considered $\pi^{+}$. The two right plots show the fit for the central region for $\mu+3 \sigma$ (top) and $\mu-3 \sigma$ (bottom), where $\mu$ and $\sigma$ are respectively the mean and the standard deviation of a Gaussian fit over each momentum bin. The left bottom represents $\mu$ for each momentum bin.

The large peak at $\sim 0.02 \mathrm{GeV}^{2} / c^{4}$ corresponds to the pion, the peak around $\sim 0.9 \mathrm{GeV}^{2} / c^{4}$ in the positive corresponds to the protons. The red distribution corresponds to the mass-squared distribution before the $\Delta \beta$ cut. A small peak around $\sim 0.27 \mathrm{GeV}^{2} / c^{4}$ can be identified as $K^{+}$background ${ }^{4}$.

\footnotetext{
${ }^{4}$ This peak can also be seen in the negative particles distribution, but it was largely attenuated after the second skim filter was applied.
} 

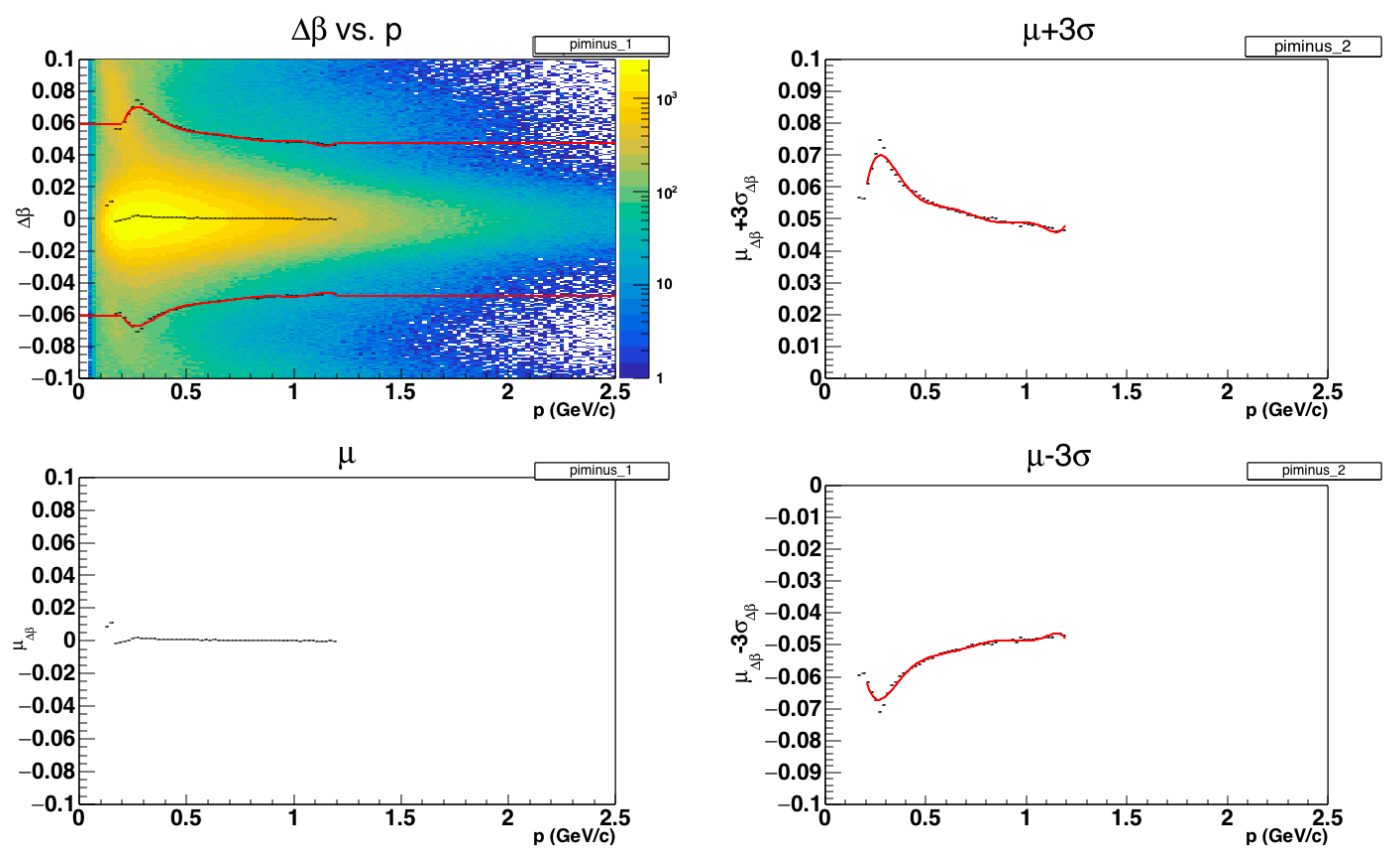

Fig. 4.3. $\pi^{-}$identification. Top left figure is the $\Delta \beta$ vs p plot. The events between the red curves, are considered $\pi^{-}$. The two right plots show the fit for the central region for $\mu+3 \sigma$ (top) and $\mu-3 \sigma$ (bottom), where $\mu$ and $\sigma$ are respectively the mean and the standard deviation of a Gaussian fit over each momentum bin. The left bottom is the mean value for each momentum bin.

\subsubsection{Neutral Particle Identification}

Tracks with positive EC status and DC status $=0$ were considered neutral particles. The separation of photons and neutrons is based on their relative velocities,

$$
\beta_{\mathrm{EVNT}}^{(n)}=\frac{l_{\mathrm{EC}}}{t_{\mathrm{EC}}-t_{\gamma}}
$$

where $l_{\mathrm{EC}}$ and $t_{\mathrm{EC}}$ are the path length and the time information from the EC, and $t_{\gamma}$, defined in equation (4.7), is the vertex time of the incoming photon. The built-in particle identification from CLAS assumes neutral particles to be photons if $\beta_{E V N T}^{(n)}>0.95$ and its energy was considered to be $p_{E V N T}=E_{\mathrm{EC}} / c$ with $E_{\mathrm{EC}}=E_{\text {tot }} / 0.273 . E_{\text {tot }}$ is the total energy deposited in the calorimeter, and 

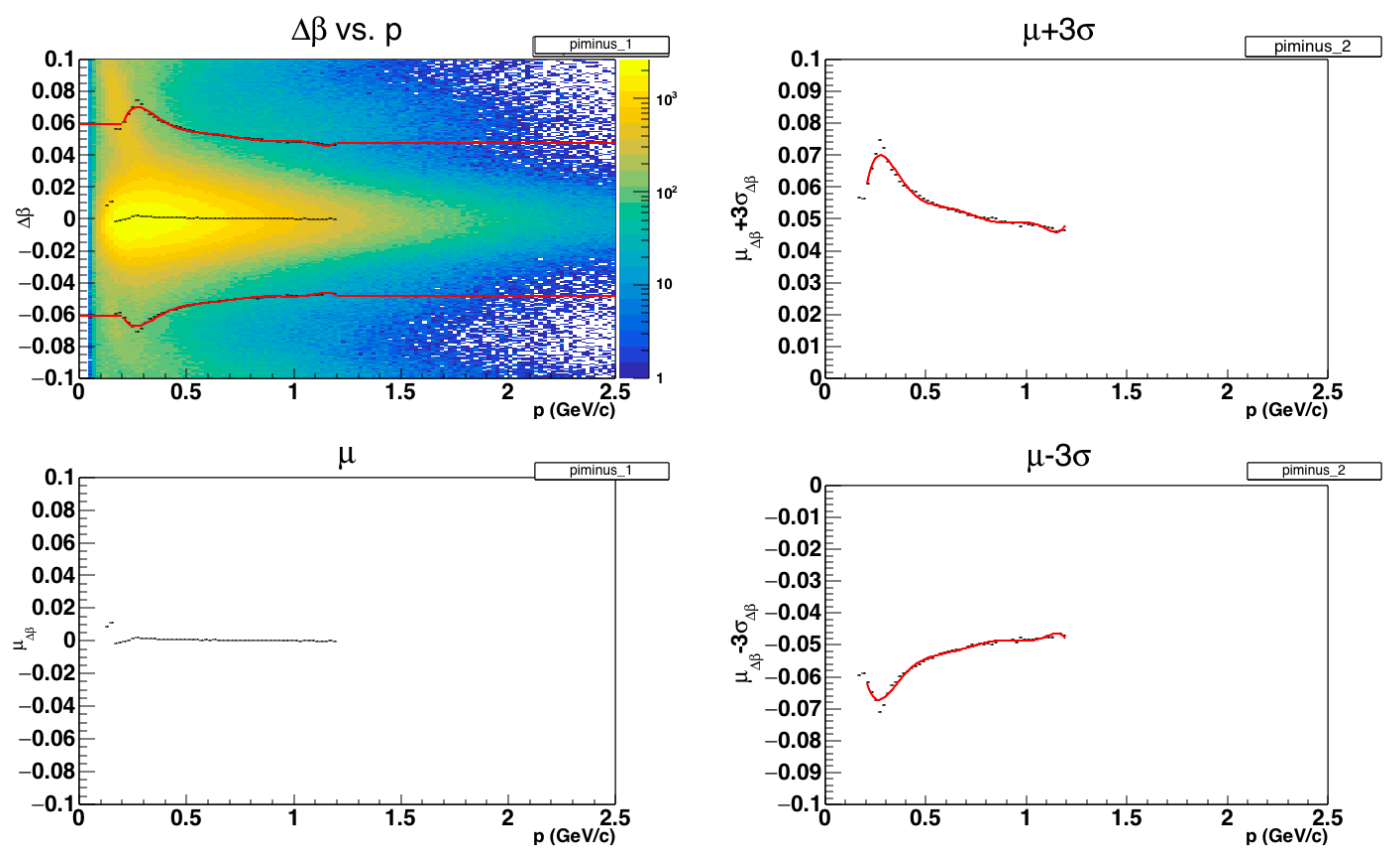

Fig. 4.4. $\pi^{+}$identification. Top left figure is the $\Delta \beta$ vs p plot. The events between the red curves, are considered $\pi^{+}$. The two right plots show the fit for the central region for $\mu+3 \sigma$ (top) and $\mu-3 \sigma$ (bottom), where $\mu$ and $\sigma$ are respectively the mean and the standard deviation of a Gaussian fit over each momentum bin. The left bottom represents $\mu$ for each momentum bin.

the constant is the sampling fraction explained in section 3.3.5. Figures 4.7 and 4.8 show the $\beta^{(n)}$ distribution for the two neutral tracks. In the left plot, the broad distribution of the sharp peak around $\beta \sim 0.9$ corresponds to the photons, while the broad distribution peaking at 0.8 to the left is associated with neutrons. The second plot to the right has a lower contamination from neutrons. This may be due to the order in which the particles are written in the bank. For this work, all the good neutral tracks with $\beta_{\mathrm{EVNT}}>0.95$ were assumed to be photons. A couple of corrections to the information of the neutral tracks were applied:

- Energy Correction. The momentum calculation is sensitive to the determination of the energy. For this analysis, we chose in the ECPB bank the maximum value between $E_{\mathrm{TOT}}$ and $E_{\mathrm{IN}}+E_{\mathrm{OUT}}$ 


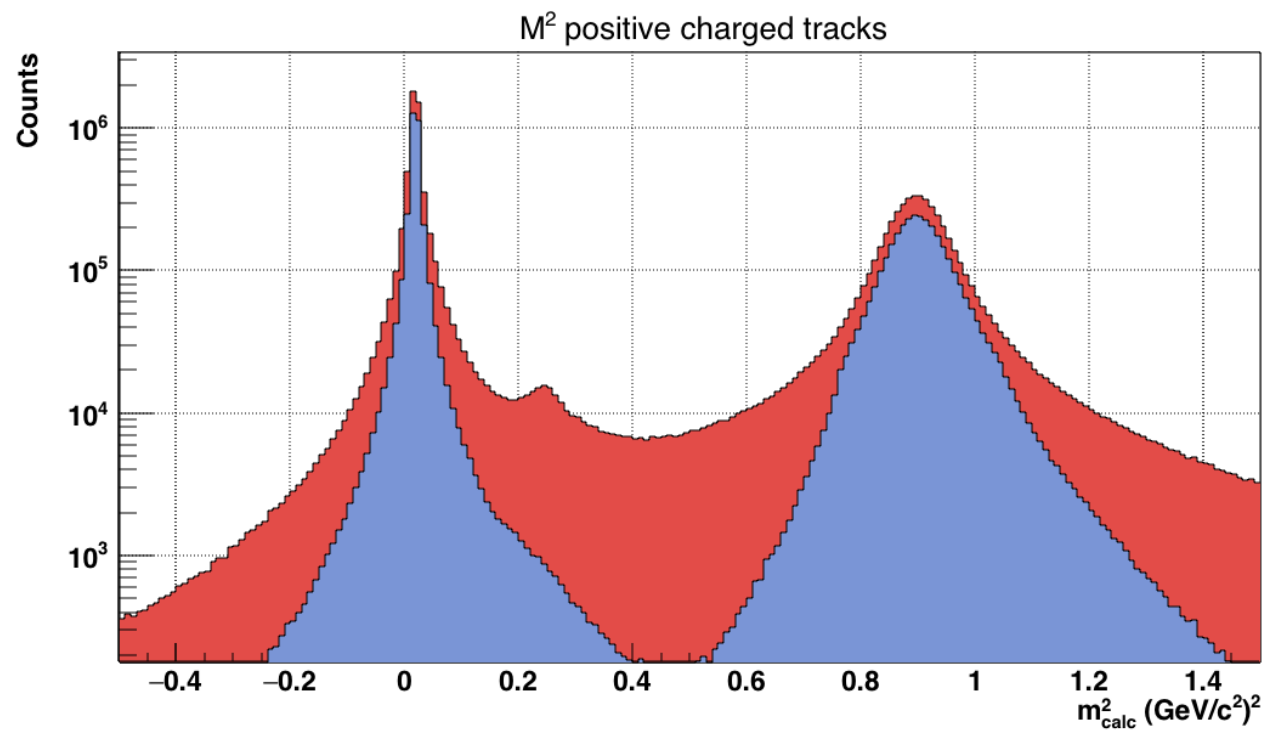

Fig. 4.5. Rest mass squared distribution for positive particles. In red the distribution before $\Delta \beta$ cut and in blue after the $\Delta \beta$ cut. For further explanation refer to the text.

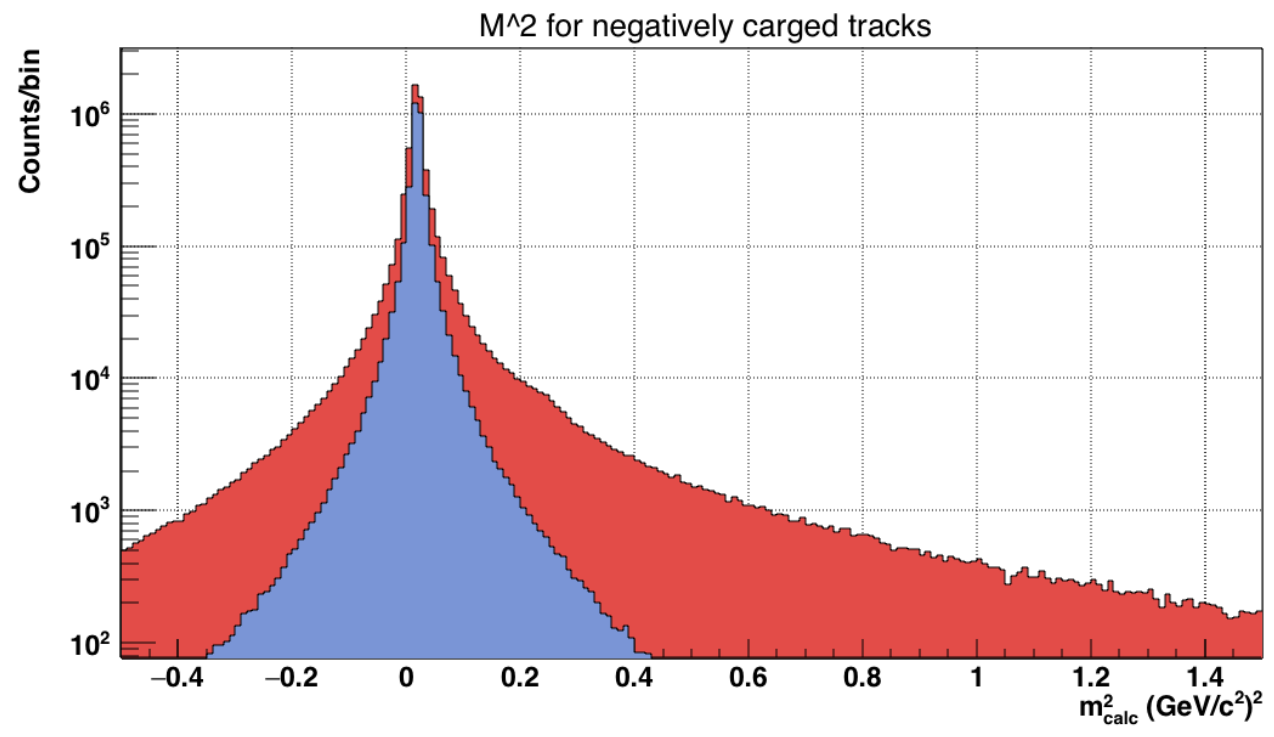

Fig. 4.6. Rest mass squared distribution for negative particles. In red the distribution before $\Delta \beta$ cut and in blue after the $\Delta \beta$ cut. For further explanation refer to the text. 


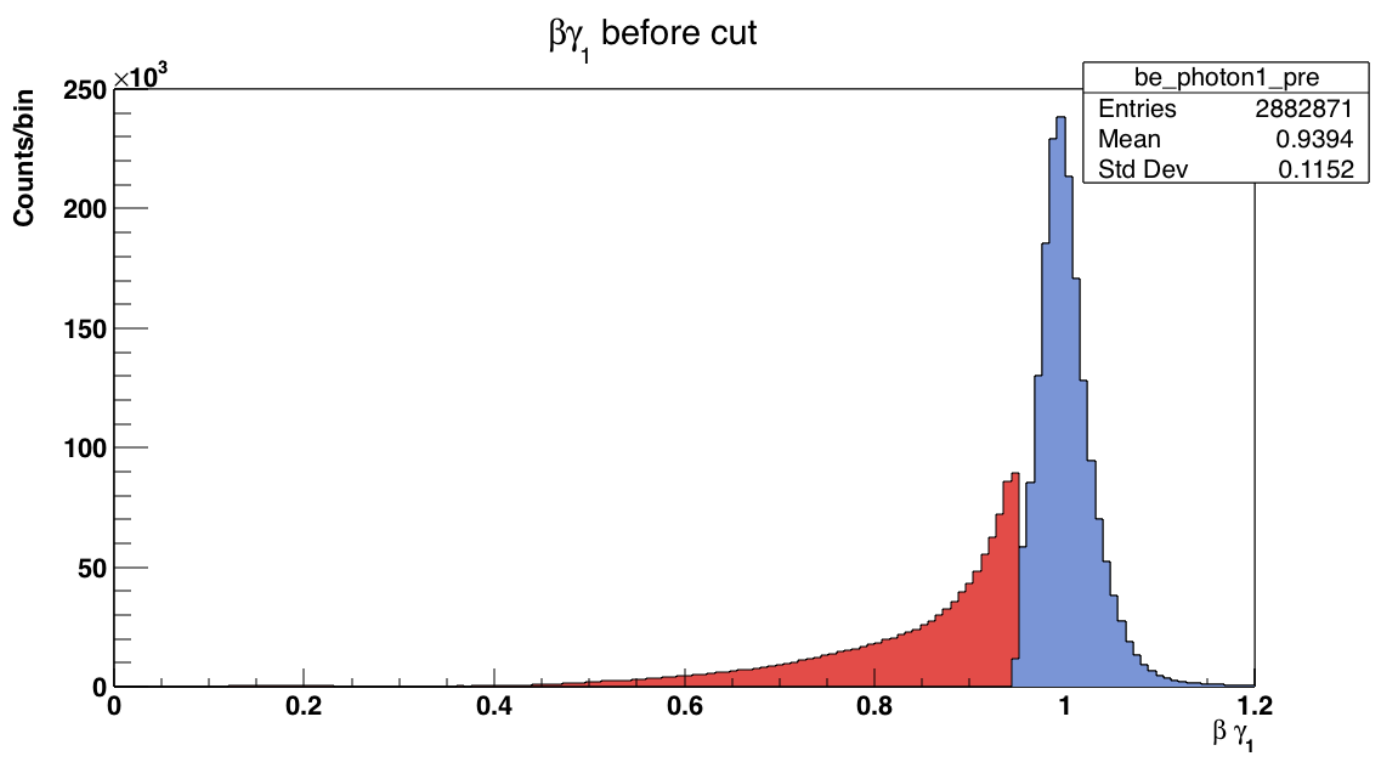

Fig. 4.7. $\beta_{\gamma_{1}}$. The events in blue are those that are considered photons, while the distribution in red is cut out.

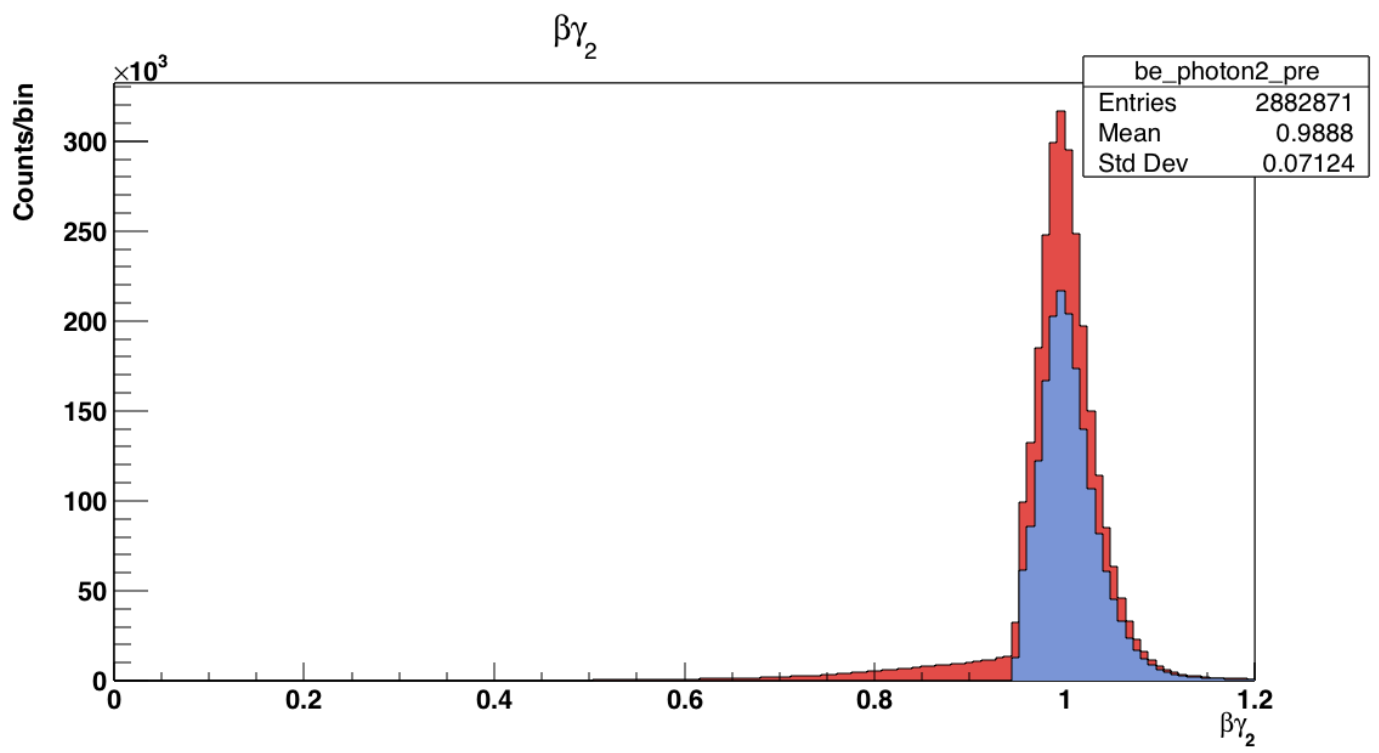

Fig. 4.8. $\beta_{\gamma_{2}}$. The events in blue are those that are considered photons, while the distribution in red is cut out. 
- Path Length Correction. The $\beta$ as well as $\theta$ and $\phi$ are sensitive to the determination of the path length. This information was directly recalculated using the MVRT bank for the interaction vertex and the information of the hit position from the ECPB bank.

\subsection{Incoming Photon Identification}

After obtaining the information on the properties of the particles in the final state, it is necessary to select the correct incoming photon that started the reaction. The photon beam produced via bremsstrahlung (as explained in 3.2) has the same 2.004 ns structure as has the electron beam. In particular, for the g13b experiment, an average of 14 electrons were detected in the tagger per trigger. These in-time electrons produce an average of 14 candidate photons as can be seen in figure 4.9. Other possible sources of electron hits in the tagger can come from random background accidentals.

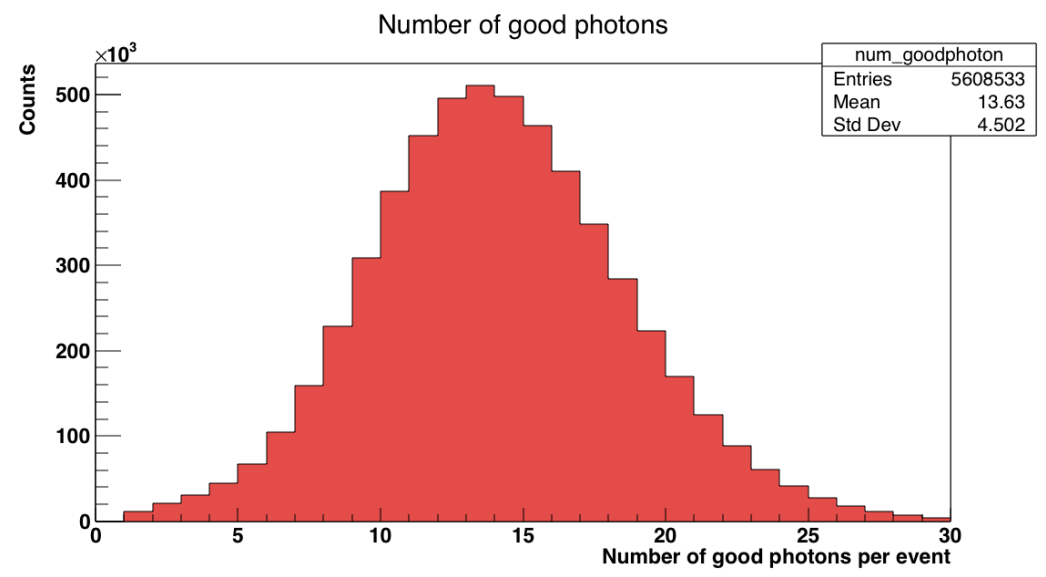

Fig. 4.9. Number of good photons per event.

The incident photon was identified by matching its time vertex, $t_{\gamma}$, with the time vertex of the particles, $t_{h}$. The calculation of the time of the hadron and 
the photon is as follows,

$$
\begin{aligned}
& t_{\gamma}=t_{\mathrm{tag}}+\frac{z+z_{\mathrm{off}}}{c}, \\
& t_{\mathrm{h}}=t_{\mathrm{sub}}-\frac{l_{\mathrm{sub}}}{c \beta_{\mathrm{calc}}}
\end{aligned}
$$

where $t_{\text {sub }}$ and $l_{\text {sub }}$ are respectively the time stamp and length from either the start counter $(\mathrm{SC})$ or the time of flight (TOF) subsystem. The $z_{\text {off }}=20 \mathrm{~cm}$ is the offset between the center point of the CLAS detector and the center of the target. The distribution of $\Delta t_{\gamma \mathrm{h}} \equiv t_{\gamma}-t_{\mathrm{h}}$ shows a big peak centered around zero and several small peaks every 2 ns corresponding mainly to events coming from different RF bunches. To select the incoming photon candidates, a cut of $\left|\Delta t_{\gamma \mathrm{h}}\right|<1$ ns was applied to the data in figure 4.10.

Additionally, some events had more than one photon in the same RF bunch as shown in figure 4.12. Only events with one photon in the same RF bunch were selected to avoid ambiguity. Before any cut, the estimate of the number of events with just one photon is $\sim 68 \%$.

\subsection{Hadron Time Coincidence}

The majority of the background from out-of-time particles was removed with the $\Delta \beta$ momentum-dependent cut. However, some of these particles lay underneath the big peak around $\Delta \beta=0$. This can be seen in the $\Delta \beta$ vs momentum plot as bands that cross the distribution centered at zero. In order to ensure that all the charged hadrons were coming from the same event, we did a cut for the time 


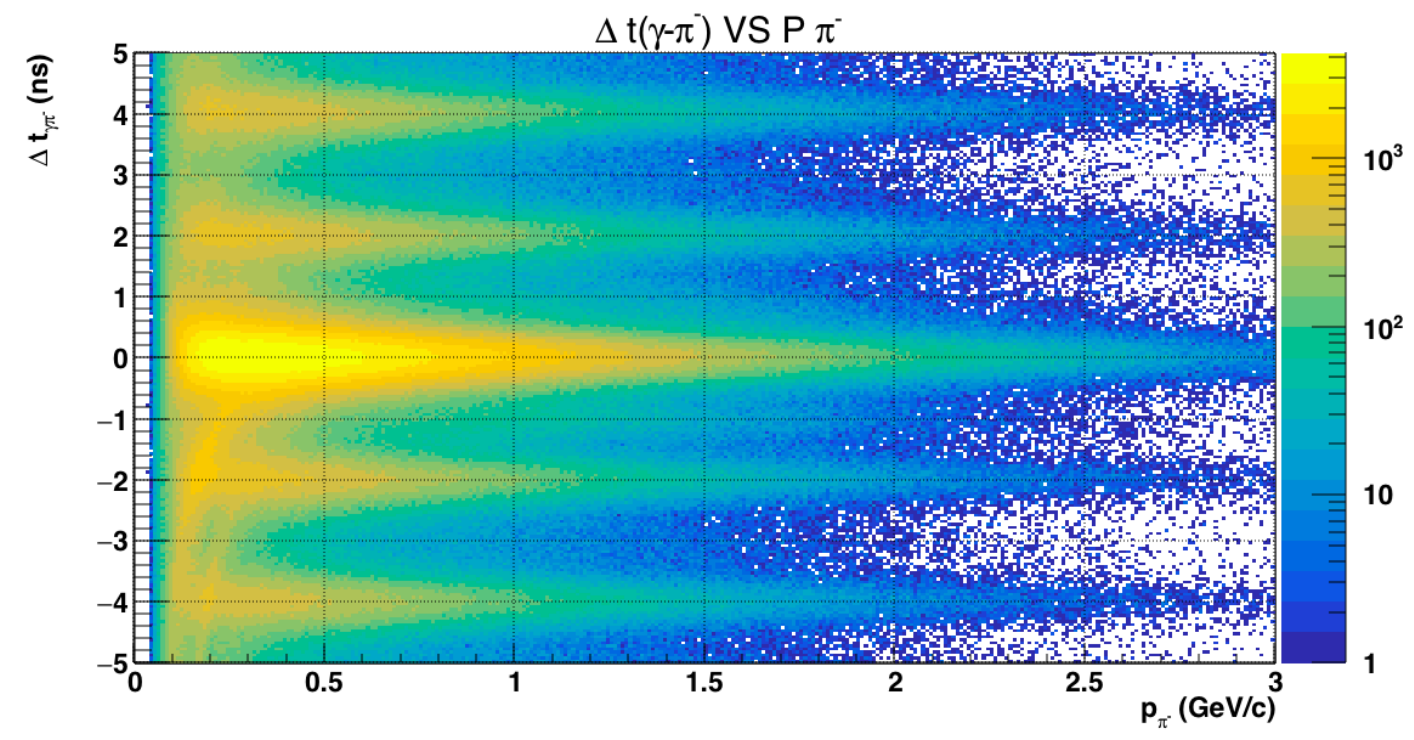

Fig. 4.10. $\Delta t_{\gamma \pi^{-}}$as a function of the $\pi^{-}$momentum. The RF structure every 2 ns is clear in the figure.

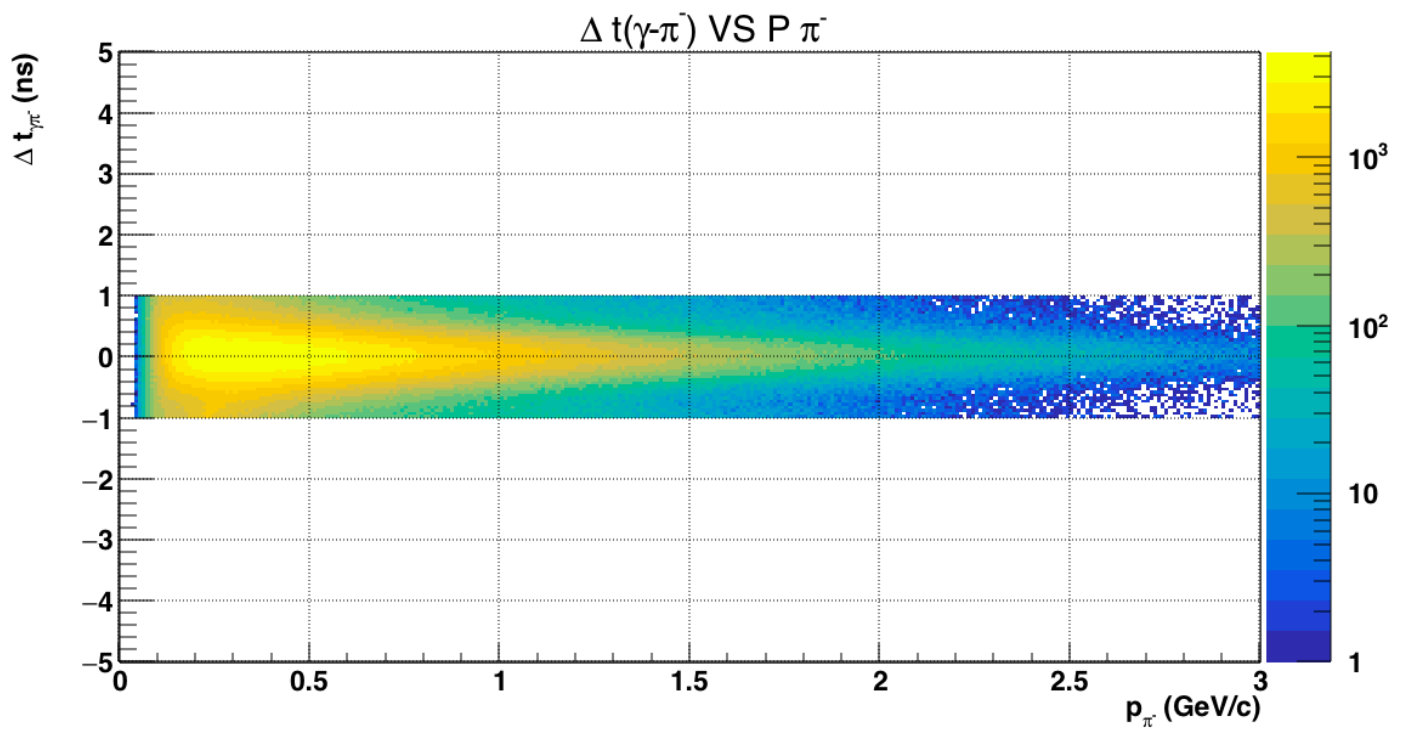

Fig. 4.11. $\Delta t_{\gamma \pi^{-}}$as a function of the $\pi^{-}$momentum after cuts.

differences of the charged tracks with respect to the $\pi^{-}$,

$$
\Delta t_{h \pi^{-}} \equiv t_{h}-t_{\pi^{-}}
$$




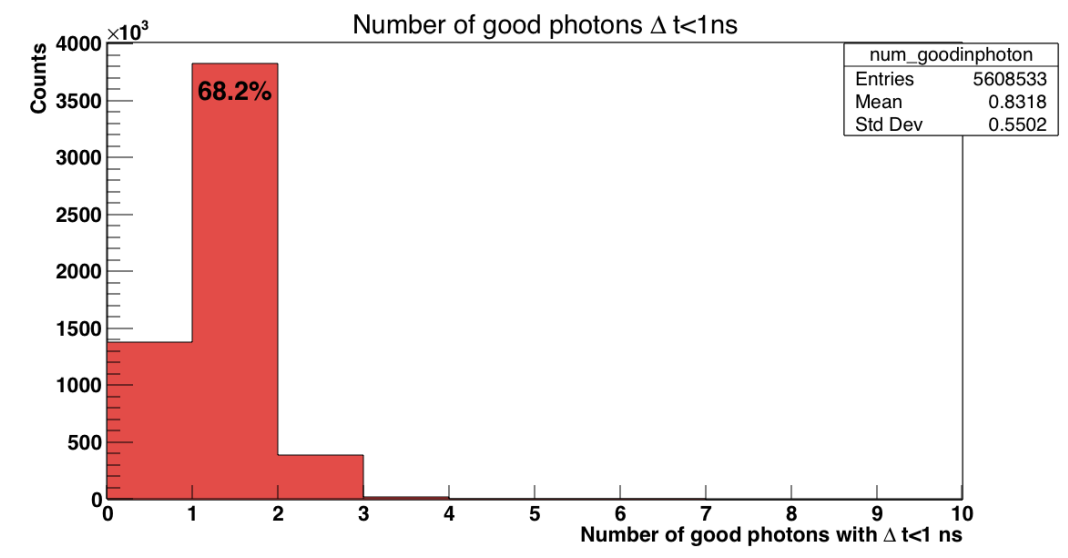

Fig. 4.12. Number of good photons per event that are in the same RF bucket, that is, $|\Delta t|<1$ ns.

with $t_{h}$ the time of the hadron defined in equation (4.7). The cut was $\left|\Delta t_{\pi^{-}}\right|<$ $1.2 \mathrm{~ns}$ and $\left|\Delta t_{\pi^{-} \pi^{+}}\right|<1.2 \mathrm{~ns}$. In figure 4.13, we can see the two cuts performed simultaneously marked by the red lines. The time of the $\pi^{-}$hit was taken as a reference time ${ }^{5}$, because having a negative polarity on the torus optimized $\pi^{-}$ identification at low momentum.

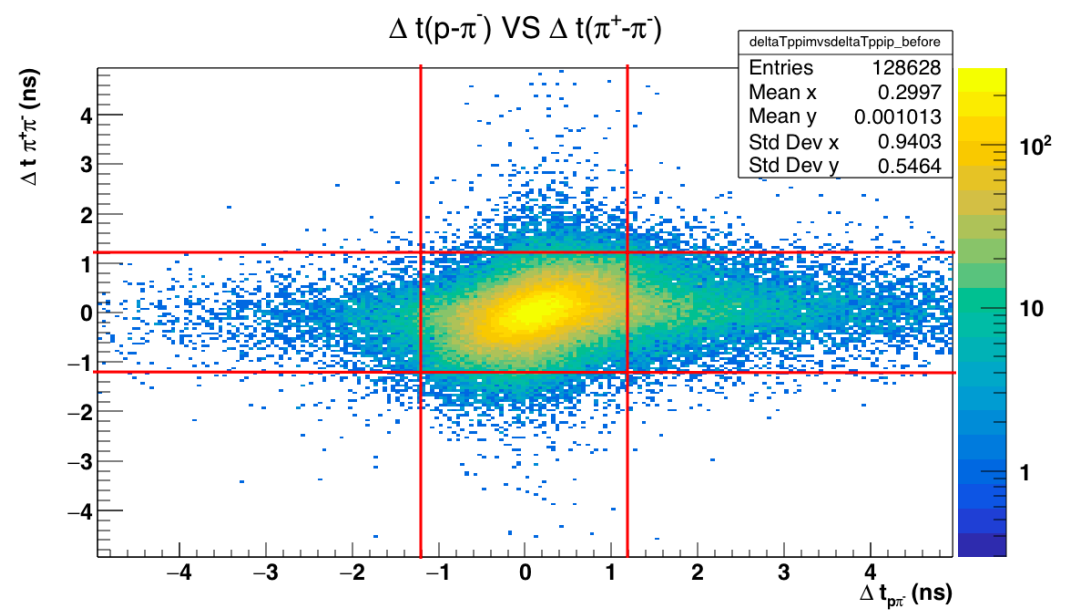

Fig. 4.13. 2-D plot of $\Delta t_{p \pi^{-}}$vs $\Delta t_{\pi^{+} \pi^{-}}$. The red lines represent the cut performed $\left|\Delta t_{h \pi^{-}}\right|<1.2 \mathrm{~ns}$.

\footnotetext{
${ }^{5}$ All the $\Delta t$ were calculated with respect to $t_{\pi^{-}}$.
} 


\subsection{Vertex Cut}

The vertex information was taken from the MVRT bank, which calculates the averaged reaction vertex using the information of all charged particles present in the event. This bank is very accurate when two or more tracks come from the primary vertex. The higher the number of tracks, the more accurate the calculation of the event vertex. The lifetime of the $\omega$ meson as reported by the

particle data group [5] is $7.75 \pm 0.07 \times 10^{-23} \mathrm{~s}$; this means that the assumption of the two charged pions coming from the primary vertex is good. In the figure 4.14 is shown the $z$ coordinate from the MVRT bank. The events in red are the events excluded after this cut. No other cut was done in the $x$ or $y$ coordinate as seen in figure 4.15 .

\section{$4.9 \quad \pi^{0}$ Reconstruction}

The reconstruction of $\pi^{0}$ was done by calculating the invariant mass squared of the two photons, given by the expression,

$$
m^{2}=\frac{2 E_{\gamma 1} E_{\gamma 2}}{c^{4}}(1-\cos \alpha)
$$

Where $\alpha$ is the angle between the two photons, and $E_{\gamma 1(2)}$ the energy in the lab frame of the photon 1(2). The invariant mass squared distribution can be seen in figure 4.16. In this plot, a clear peak can be seen around the nominal value of the $\pi^{0}$ mass squared over a smooth background. There is also a peak at zero, which represents a nonresonant background, that is, two photons that are produced in the decay, but do not come from a $\pi^{0}$. The $\pi^{0}$ events are selected by fitting a Gaussian distribution to the $m^{2}(\gamma \gamma)$ and applying a $3 \sigma$ cut around the 


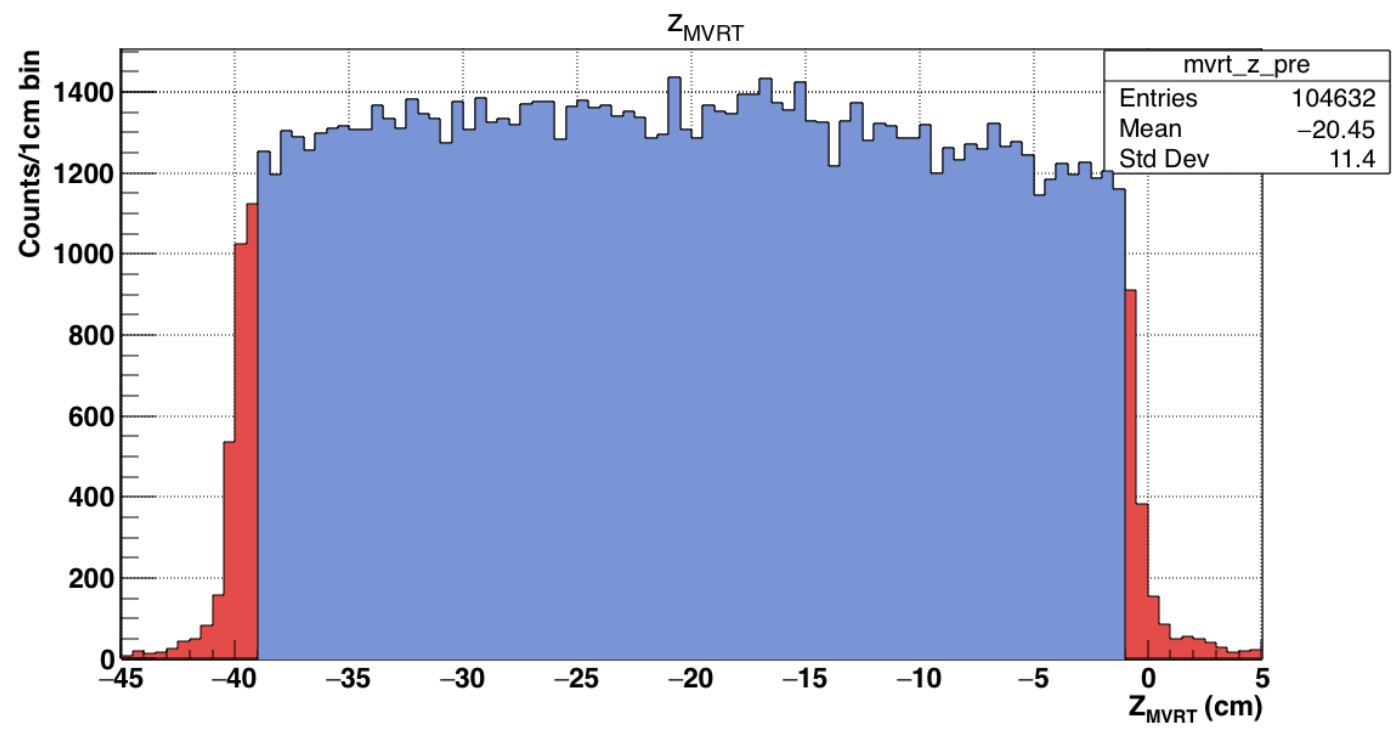

Fig. 4.14. $z$ coordinate, with respect to the center of CLAS, of the event vertex as found in the MVRT bank. The vast majority of the events are produced within the nominal values of the target. The events in red are those that are cut out of the data sample.

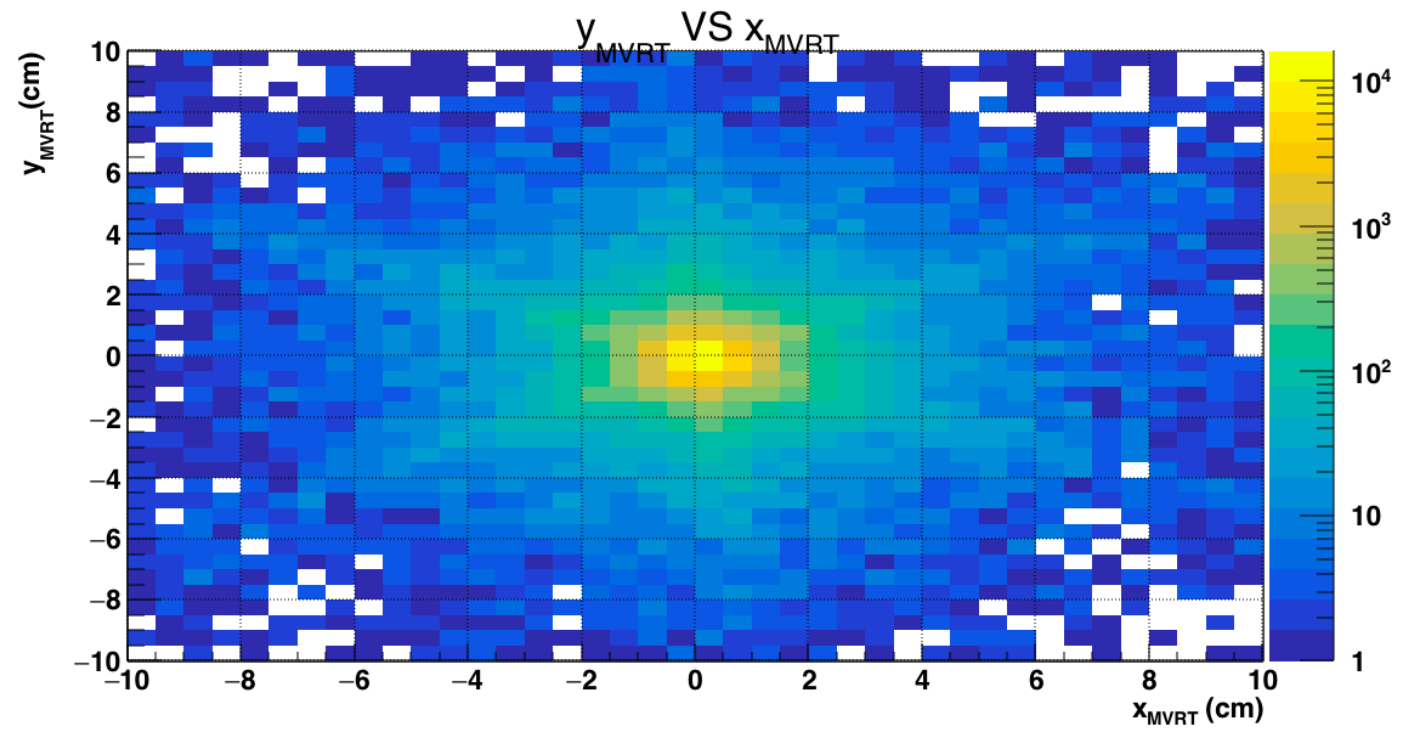

Fig. 4.15. $y$ vs. $x$ coordinate for the primary vertex from MVRT bank. No cut was performed for these quantities. 
distribution centroid. The centroid is shifted $\sim 0.0007 \mathrm{GeV}^{2} / \mathrm{c}^{4}$ with respect to the nominal value.

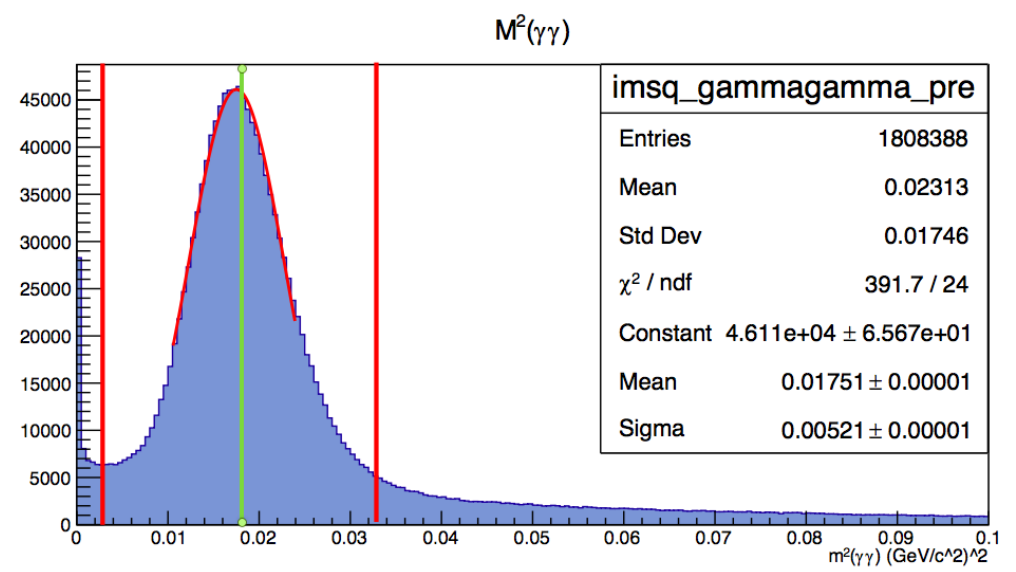

Fig. 4.16. Invariant mass squared of the two photons. The red lines mark the cut. The green line indicates the position of the nominal value of the $m_{\pi^{0}}^{2}=$ $0.01822 \mathrm{GeV}^{2} / \mathrm{c}^{4}$

\subsection{Fiducial Cuts}

The efficiency and reliability of the track reconstruction are reduced at the edges of the drift chambers. It is also possible that the charged particles that hit the support frames or the cryostats of the torus escaped detection completely. On the other hand, the magnetic field close to the cryostats' surfaces is not uniform, making the task of mapping and modeling very difficult. Tracks coming from those regions are associated with more significant systematic uncertainties in the measured momentum. This uncertainty will propagate in the calculation of the physical observables, increasing also their uncertainty. For these reasons, fiducial cuts are applied to ensure the tracks are more reliable. The code used to perform these cuts was developed by a collaborator, Paul Mattione, for the g13 group. 


\subsection{Energy and Momentum Corrections}

\subsubsection{Energy Loss Corrections}

Charged particles passing through a material, other than electrons and positrons, lose energy primarily by ionization. The atomic electron collisions are in nature a statistical phenomenon, but given that the number of collisions per path length is large, the fluctuations in the total energy loss are small. This means that it is possible to describe the macroscopic behavior by defining the average energy loss per unity length, called stopping power $\frac{d E}{d x}$ [40]. The stopping power is described by the Bethe-Bloch formula. In particular, the charged particles in CLAS lose energy in their path from the primary vertex, passing through the target material and target cell structure, the beam pipe, supporting structures, the start counter, and the air gap between the start counter, and region one of the drift chambers. From region one to region three, the reconstruction software calculates the momentum $p_{\text {meas }}$ without taking into account the previous energy loss. The four-momentum is found by multiplying the measured momentum and a factor which is different for each particle ${ }^{6}$ :

$$
\begin{gathered}
P_{p}^{\mathrm{Eloss}}=\zeta_{p} P_{p}^{\mathrm{EVNT}}, \\
P_{\pi^{+}}^{\mathrm{Eloss}}=\zeta_{\pi^{+}} P_{\pi^{+}}^{\mathrm{EVNT}}, \\
P_{\pi^{-}}^{\mathrm{Eloss}}=\zeta_{\pi^{-}} P_{\pi^{-}}^{\mathrm{EVNT}} .
\end{gathered}
$$

$P_{i}^{\mathrm{EVNT}}$ is the momentum of the $i$ particle as found in the EVNT bank, $P_{i}^{\text {Eloss }}$ is the momentum after energy loss correction, $\zeta_{i}$ is the correction factor and $i$ is the

\footnotetext{
${ }^{6}$ The Bethe-Bloch formula does not depend on the mass of the particles, but it does depend on the momentum of the particle
} 
type of particle $\left(\pi^{+}, \pi^{-}\right.$, proton). A collaborator, Eugene Pasyuk, developed a program to account for this energy loss. The correction is on the order of a few $\mathrm{MeV}$. Figures 4.17, 4.18 and 4.19 give the relative correction, $\Delta p / p$, for protons, $\pi^{+}$, and $\pi^{-}$respectively.

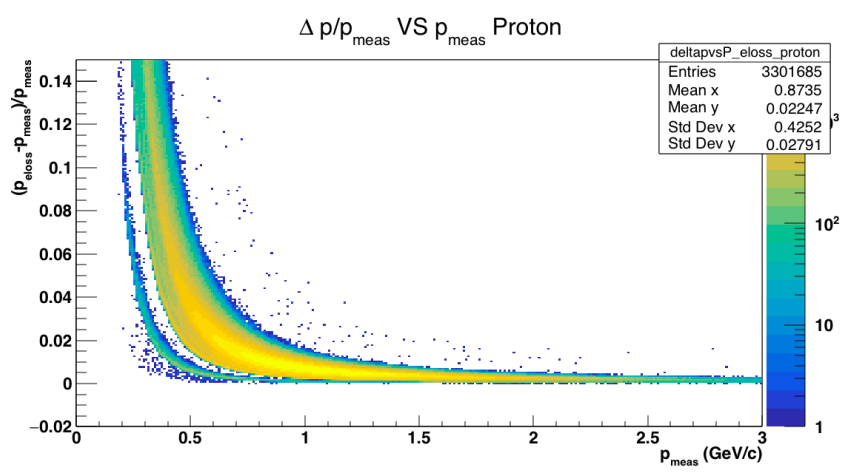

Fig. 4.17. Relative momentum difference after energy loss $\Delta p / p_{\text {meas }}$ for the proton after particle identification cuts.

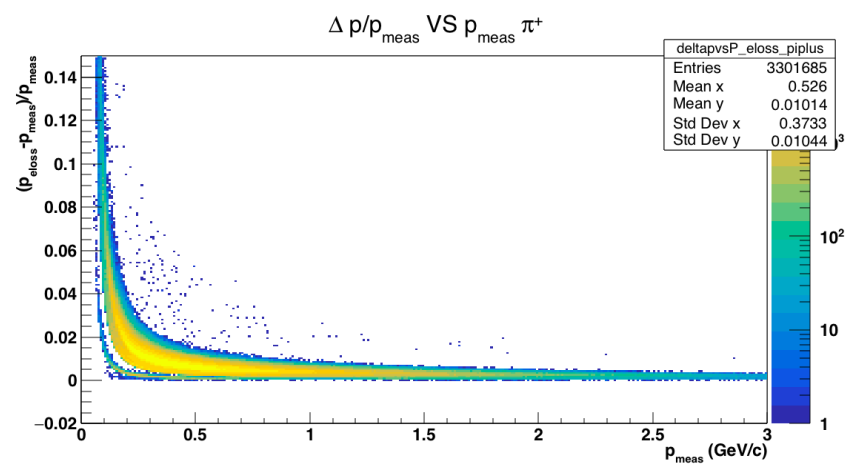

Fig. 4.18. Relative momentum difference after energy loss $\Delta p / p_{\text {meas }}$ for the tracks identified as $\pi^{+}$after particle identification cuts.

\subsubsection{Momentum Corrections}

After applying the energy loss corrections, momentum corrections for the charged particles were applied. These corrections account for geometrical drift chambers misalignments and inaccuracies for the toroidal magnetic field map. The correc- 


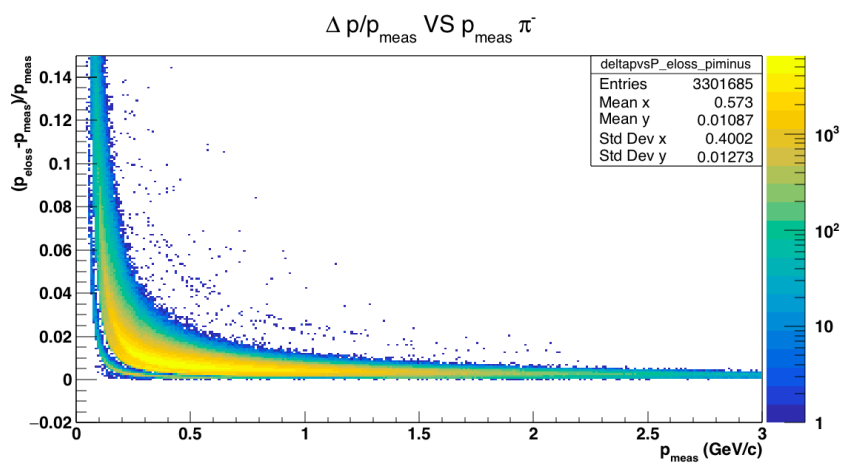

Fig. 4.19. Relative momentum difference after energy loss $\Delta p / p_{\text {meas }}$ for the tracks identified as $\pi^{-}$after particle identification cuts.

tions were calculated by applying kinematic fitting (see reference [41] ) of the reaction $\gamma d \rightarrow p p \pi^{-}$using data from g13a. The case in which all the tracks were detected was compared with two topologies: $\gamma d \rightarrow p p\left(\pi^{-}\right)$and $\gamma d \rightarrow p \pi^{-}(p)$. The information from the missing particle (denoted as $(X)$ ) was obtained via energy and momentum balance. The corrections were then determined by calculating the mean energy loss corrected tracks and the momentum of the missing particle. The error in the measurement of the track was largest for low-momentum protons. The corrections applied to the subset of events with coherent peak of $2.3 \mathrm{GeV}$ is shown in the figures $4.20,4.21$, and 4.22 , for the proton, $\pi^{+}$, and $\pi^{-}$ respectively. For more details, see reference [42].

\subsubsection{Incoming Photon Energy Corrections}

As previously discussed in section 3.2.4, the energy of the incoming photon is determined by knowing the energy of the electron beam and the energy of the scattered electron. The latter is determined by knowing the magnitude of the magnetic field and the hit into the physical paddle in the tagger hodoscope. A study made by one collaborator, Mike Williams (see reference [43]), noticed a 


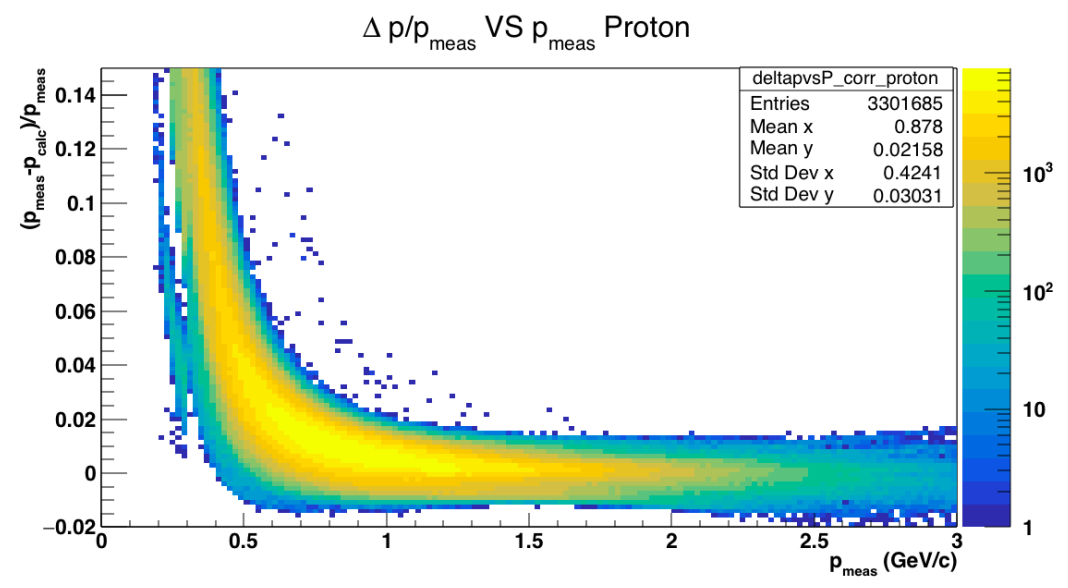

Fig. 4.20. Relative momentum difference after momentum correction $\Delta p / p_{\text {meas }}$ for the tracks identified as protons.

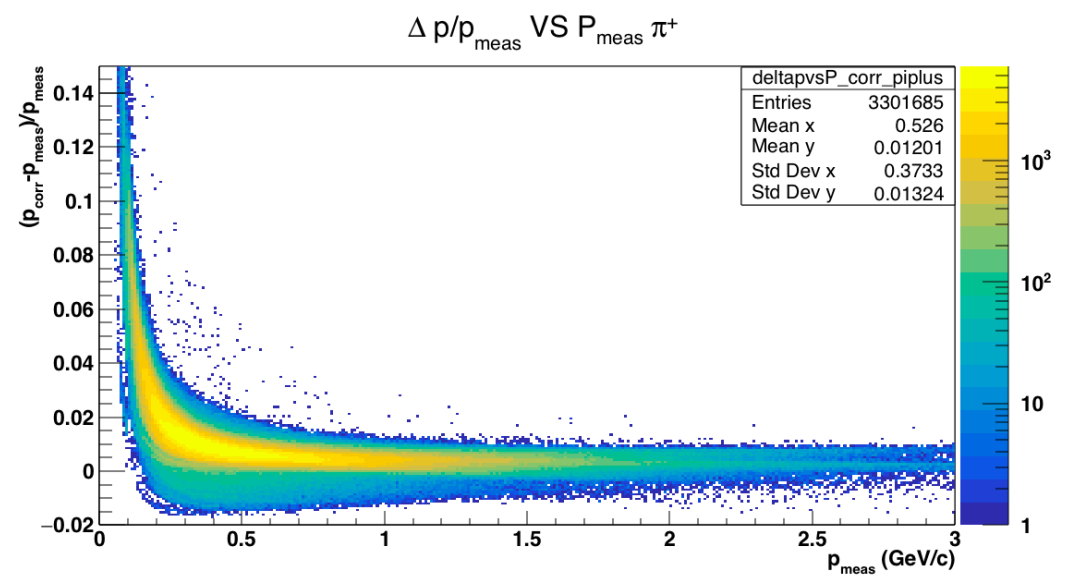

Fig. 4.21. Relative momentum difference after momentum correction $\Delta p / p_{\text {meas }}$ for the tracks identified as $\pi^{+}$.

systematic shift in the photon energy value that is compatible with a gravitational sag of some of the tagger components. The overestimate on the energy of the electron produces an underestimate of the photon energy. A multiplicative constant was calculated to correct this error and can be seen in figure 4.23. 


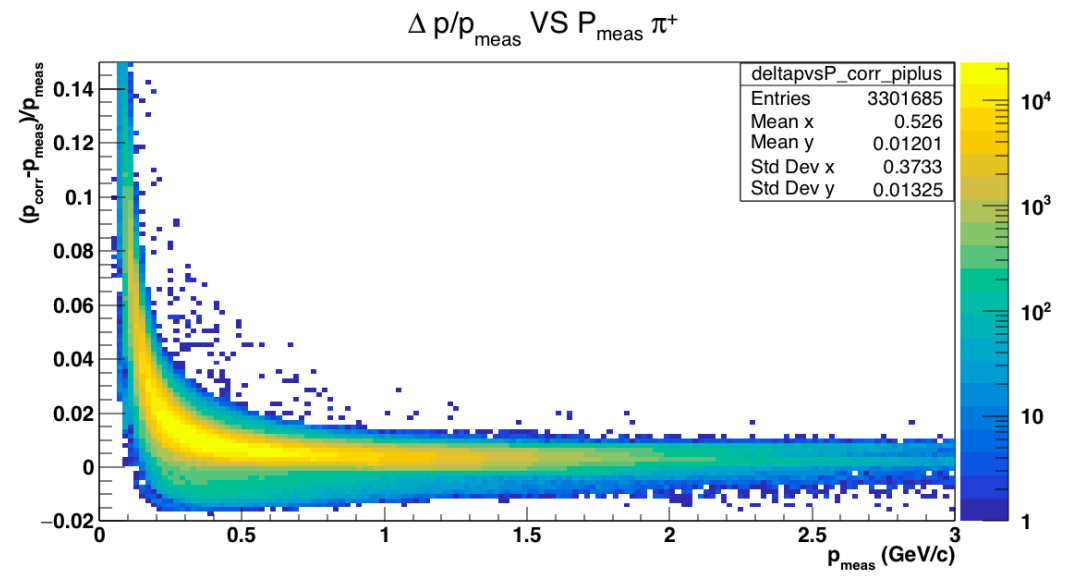

Fig. 4.22. Relative momentum difference after momentum correction $\Delta p / p_{\text {meas }}$ for the tracks identified as $\pi^{-}$.

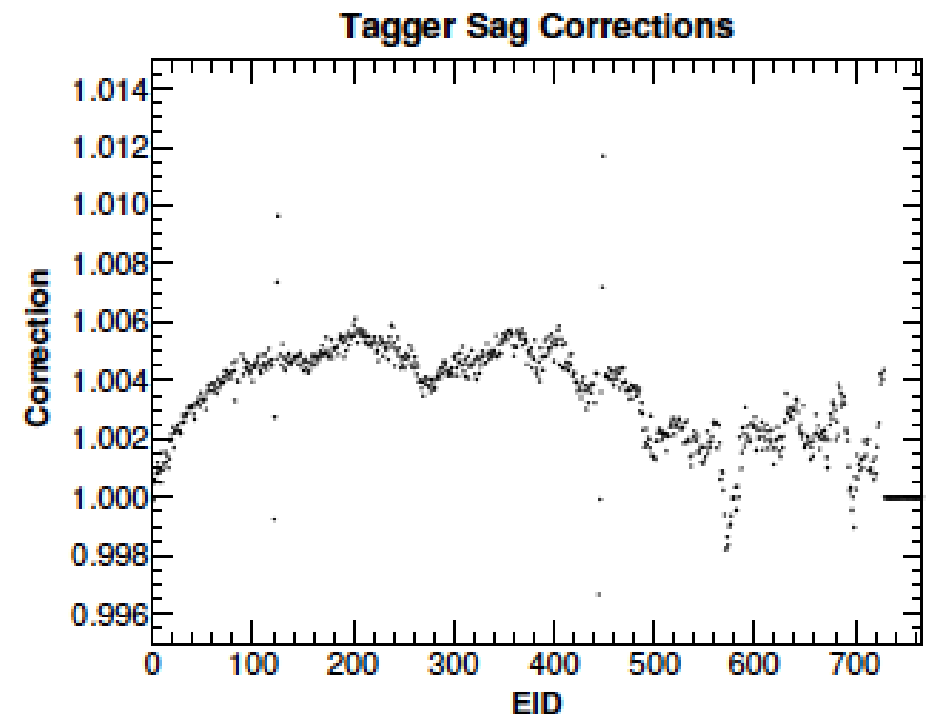

Fig. 4.23. Tagger correction accounting for the gravitational sag.

\subsection{Missing Momentum Cut}

The reaction $\vec{\gamma} d \rightarrow p \omega(n)$ is identified by using the kinematic information of all the final-state particles. The only particle that is not detected, hence the particle that is "missing", is the spectator neutron, and its kinematics can be found by conservation of four momentum. The missing momentum $p_{X}$ is calculated as 
follows,

$$
p_{X}=\left|\vec{p}_{\gamma}-\left(\vec{p}_{p}+\vec{p}_{\pi^{+}}+\vec{p}_{\pi^{-}}+\vec{p}_{\gamma_{1}}+\vec{p}_{\gamma_{2}}\right)\right|
$$

where $\vec{p}_{i}$ is the momentum of the detected particles after momentum and Eloss corrections.

The signal events ${ }^{7}$ that are considered so far can come from different sources; one is the quasi-free $(\mathrm{QF}) \omega$ photoproduction, and, on the other hand, processes dominated by final-state interactions. The QF channel, which is the one that is considered in this work, assumes that the spectator neutron does not interact with any of the final state particles. What this means is that the momentum of the spectator will be the Fermi momentum that it had inside the deuteron at the moment of the interaction. By applying a missing-momentum cut for $P_{X}\left(\gamma d \rightarrow p \pi^{+} \pi^{-} \gamma \gamma X\right) \leq 0.2 \mathrm{GeV} / c$, the final state interaction contributions are reduced.

\section{$4.13 \omega$ Meson Reconstruction}

After all the cuts described in this chapter, it is important to separate the background events from the signal events. This separation was achieved by analyzing the invariant mass squared of the three pions $m^{2}\left(\pi^{+} \pi^{-} \pi^{0}\right)$ and the missing mass squared $m_{X}^{2}$ simultaneously. As it is possible to see in the figure 4.26 (left), it is possible to distinguish three main regions: the signal region (the big peak), the nonresonant background, and a small peak that is $\eta$ background. It is important to notice that the nonresonant background intersects with the signal region. For each one of the photon energy subsets in g13b, we applied a missing-mass-squared

\footnotetext{
${ }^{7}$ These are events that do not come from background sources.
} 
dependent cut. The projection on missing mass squared bins was fitted with a double Gaussian as seen in figure 4.24.

Finally, the $\mu_{i} \pm 3 \sigma_{i}$ for the ith bin were then plotted and fitted with a seconddegree polynomial as seen in figure 4.25. This cut, as can be seen in figure 4.26, removes the $\eta$ background and the majority of the nonresonant background. However, there are still some events under the $\omega$ peak. This issue will be addressed in the next chapter. 

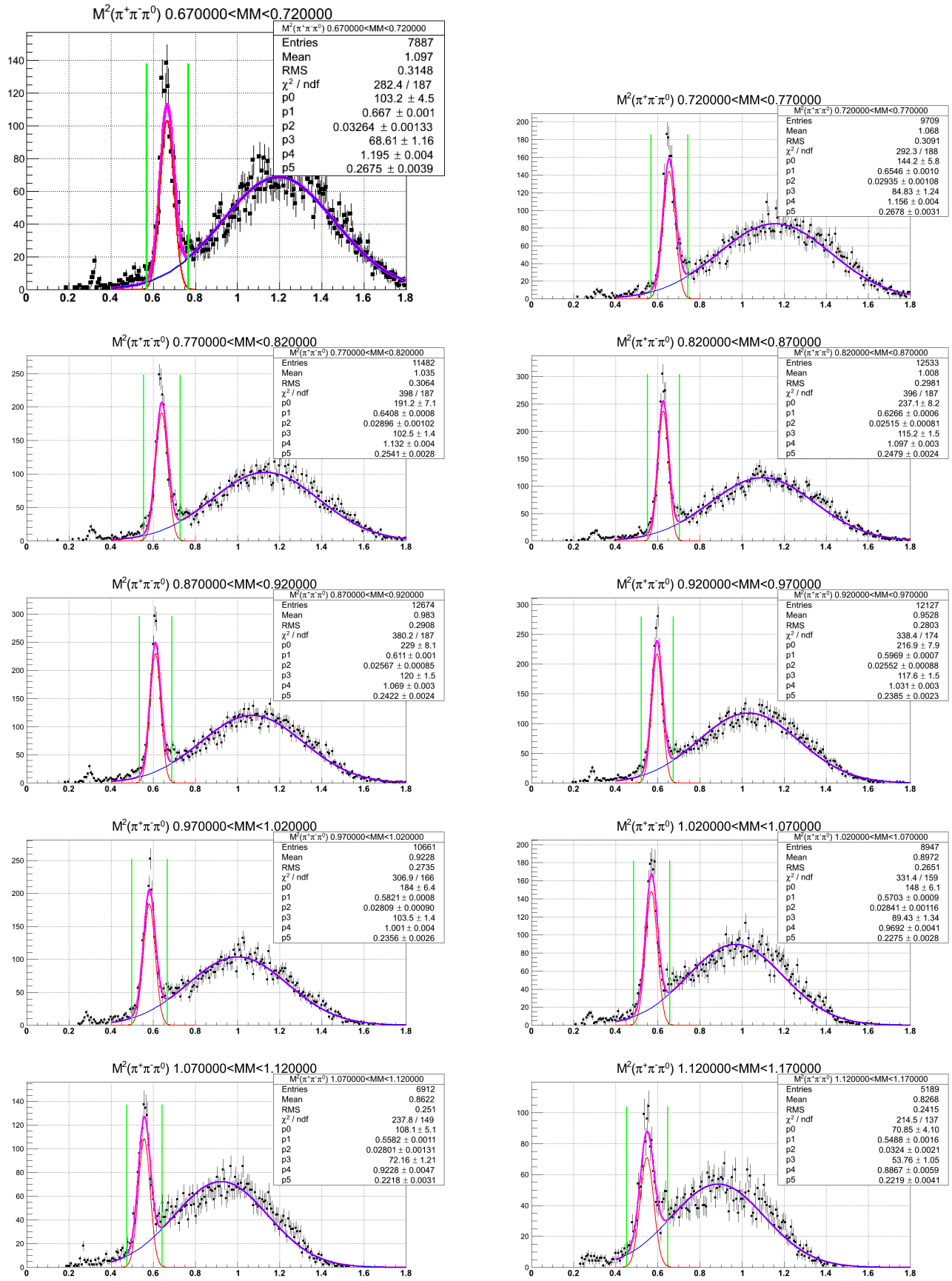

Fig. 4.24. Two-Gaussian fit to the projection of the invariant mass squared of the three pions for different missing mass squared bins. The red lines show the $3 \sigma$ cut. 

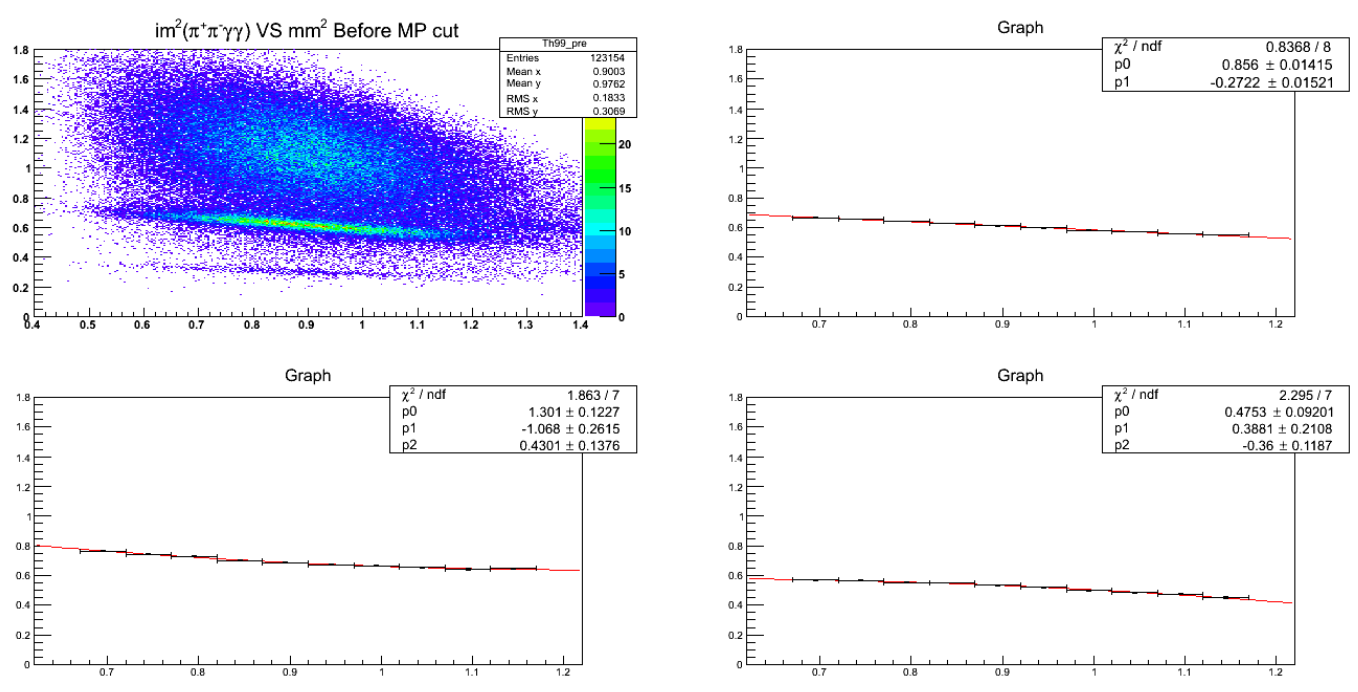

Fig. 4.25. $3 \sigma$ cut around the centroid. In the two bottom plots, it is possible to see the 2 nd degree polynomial fit to the $\mu \pm 3 \sigma$.
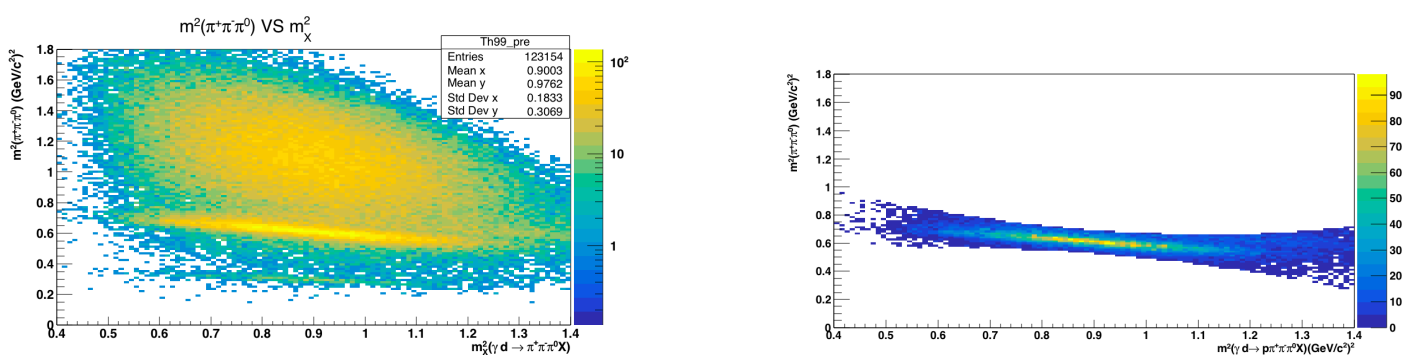

Fig. 4.26. Invariant mass squared of the three pions $m^{2}\left(\pi^{+} \pi^{-} \pi^{0}\right)$ vs. the missing mass squared $m_{X}^{2}\left(\gamma d \rightarrow p \pi^{+} \pi^{-} \pi^{0} X\right)$ before (left) and after the cut (right). This is an example for the energy set with coherent peak at $2.3 \mathrm{GeV}$. 


\section{Chapter 5}

\section{Data Analysis: Beam Asymmetry}

After selecting the candidate $\omega$ photoproduction events, we then calculated the polarization observable $\Sigma$ that parametrizes the beam spin asymmetry. For this work, we are using linearly polarized photons incident on an unpolarized target. In this chapter, the methodology for the calculation of the observable is explained in detail.

\section{$5.1 \quad$ Binning}

The kinematics of a two-body final state reaction can be completely described

by two parameters. In this work, the energy of the incoming photon $E_{\gamma}$ was chosen to be in the lab frame and the cosine of the polar angle of the $\omega$ meson in the center of momentum frame $\left(\cos \theta_{C M}^{\omega}\right)$. The $z$-axis is defined by the incoming photon direction (see figure 5.1).

Two energy bins were extracted for each coherent peak subset, with a width of $100 \mathrm{MeV}$, covering a total range from $1.1 \mathrm{GeV}$ to $2.3 \mathrm{GeV}$. Although the energy bins could overlap between subsets, this option was discarded because the degree 


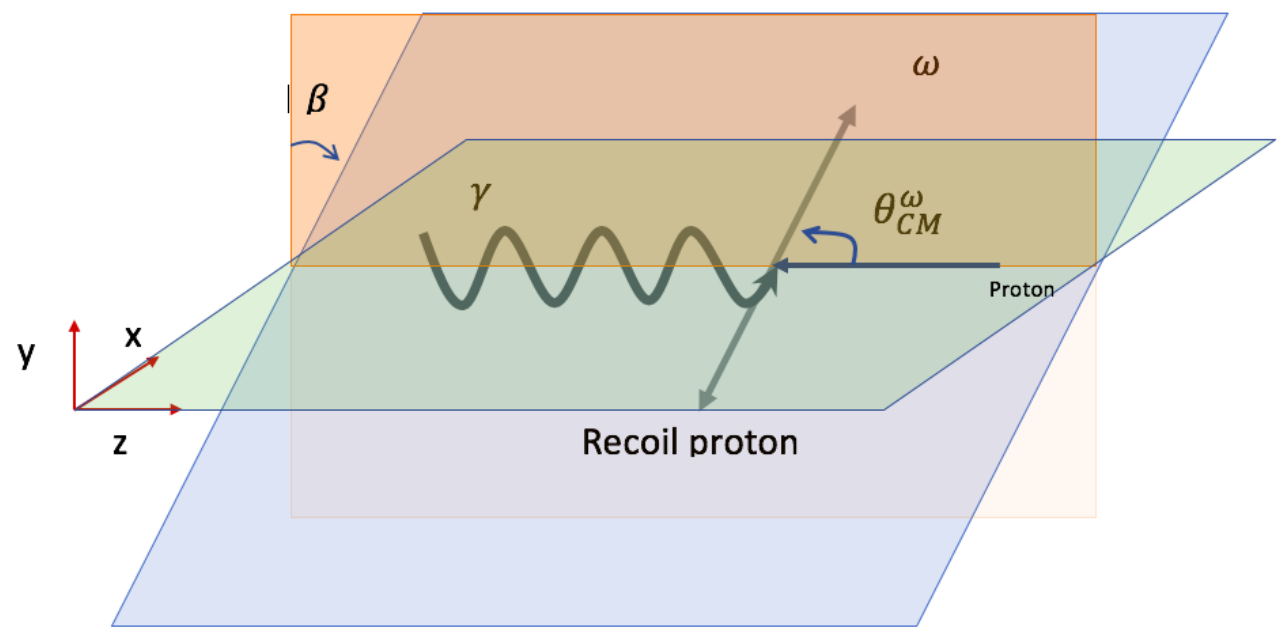

Fig. 5.1. Schematic representation of the reaction $\gamma p \rightarrow p \omega$ in the center-of-mass frame. The blue rectangle represents the decay plane, and its normal unitary vector is defined by $\hat{n}=\frac{\hat{z} \times p_{C M}^{\omega}}{\left|\hat{z} \times p_{C M}^{\omega}\right|}$. The orange rectangle represents the polarization plane for the incoming photon. The angle between the two planes is denoted as $\beta$. Finaly, the $\theta_{\mathrm{CM}}$ is the angle between the proton and the $\omega$ meson in the center-of-mass frame. The $z$-axis is the direction of the incoming photon.

of linear polarization decreases as the photon energy gets farther from the energy of the coherent edge $\left(E_{\mathrm{coh}}\right)$. In particular, the degree of photon polarization increases with the fractional photon energy $x \equiv E_{\gamma} / E_{0}$, where $E_{\gamma}$ denotes the photon energy and $E_{0}$ the electron beam energy. The figure of merit (FOM) is defined as

$$
\mathrm{FOM}=L P^{2}
$$

where $L$ is the luminosity ${ }^{1}$, and $\mathrm{P}$ is the degree of polarization of that photon. An event with lower polarization will not have as significant an impact on the

\footnotetext{
${ }^{1}$ The luminosity does not depend on the energy of the incoming photon, but on the beam energy.
} 

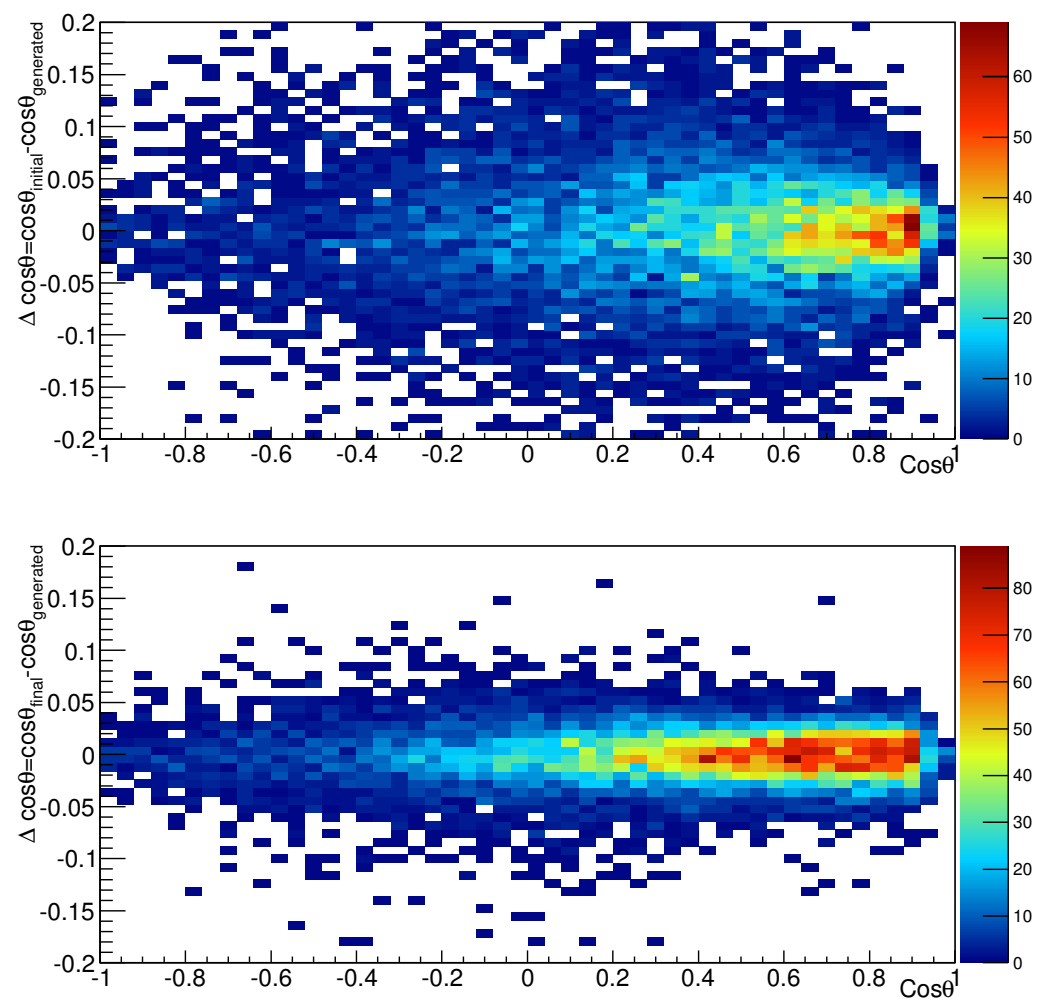

Fig. 5.2. Difference between the $\cos \theta$ generated with the Monte Carlo, and the $\cos \theta$ "detected" as a function of $\cos \theta_{\mathrm{CM}}$. The top plot takes the information of the incoming particles assuming the proton at rest. The bottom plot takes the information from the final-state particles without any assumption.

FOM.

To accurately calculate the polar and azimuthal angle in the center-of-momentum frame, the boost transformation was calculated with the final-state particles instead of the initial particles. A Monte Carlo study of the uncertainty in the kinematic variables coming from the resolution of the detectors was performed. The conclusion of this study was that the uncertainty of the kinematic variables due to the resolution of CLAS and the Fermi momentum was smaller than assuming the proton target was at rest, as can be seen in figure 5.2.

The $\cos \theta_{\mathrm{CM}}$ variable was divided in 6 bins as well. A total of 72 kinematic 
bins were calculated and are reported later in this document.

\subsection{Beam Asymmetry: the $\phi$ Binned Method}

The polarized cross section for vector mesons can be written as,

$$
\left(\frac{d \sigma}{d \Omega}\left(E_{\gamma}, \theta_{\mathrm{CM}}^{\omega}, \beta\right)\right)_{\mathrm{pol}}=\left(\frac{d \sigma}{d \Omega}\left(E_{\gamma}, \theta_{\mathrm{CM}}^{\omega}\right)\right)_{\mathrm{unpol}}(1-P \Sigma \cos (2 \beta))
$$

where $\left(\frac{d \sigma}{d \Omega}\right)_{\text {unpol }}$ is the unpolarized differential cross section, $E_{\gamma}$ is the energy of the incoming photon, $\theta_{\mathrm{CM}}^{\omega}$ is the polar angle of the $\omega$ in the center-of-mass frame (see figure (5.1)), $\mathrm{P}$ is the degree of polarization of the photon, $\beta=\phi-\varphi$ is the azimuthal angle between the polarization plane and the production plane, and $\Sigma$ is the beam asymmetry observable to be extracted; $\Sigma$ is a dimensionless quantity.

The observable $\Sigma$ can be determined in different ways: one can, for example, perform a fit to the polarized cross section with a function similar to equation (5.2). The problem with this method is that the unpolarized differential cross section would have to be known precisely. Also, a very accurate study of the acceptance would have to be done, since the $\phi$ distribution is very dependent on the CLAS acceptance. A more standard way to calculate these kinds of asymmetries is to calculate a ratio of linear combinations of the $\phi$ distribution for two orthogonal data sets (for example, PARA and PERP). ${ }^{2}$ Taking the ratio of the parallel and perpendicular distributions will serve to reduce systematic errors as it will cancel out the acceptance dependence of the detector which is not a function of the polarization.

\footnotetext{
${ }^{2}$ PARA (PERP) is short for parallel (perpendicular), which denotes the orientation of the photon electric field with respect to the floor of the hall.
} 
For this analysis, we have used the calculation of the distribution $Y$,

$$
Y \equiv \frac{\left(\frac{d N}{d \beta}\right)^{\perp}-\left(\frac{d N}{d \beta}\right)^{\|}}{\left(\frac{d N}{d \beta}\right)^{\perp}+\left(\frac{d N}{d \beta}\right)^{\|}}
$$

The azimuthal distribution of the events produced with linearly polarized photons on an unpolarized target is

$$
\left(\frac{d N}{d \beta}\right)^{\|, \perp} \sim A(\phi) F^{\|, \perp}\left(1-P^{\|, \perp} \Sigma \cos \left(2 \beta^{\|, \perp}\right)\right)
$$

where $A(\phi)$ is the acceptance of the detector, $F^{\|, \perp}$ is the flux of the incoming photons, and $P^{\|, \perp}$ is the degree of polarization. For the PARA data set, we have $\varphi=0$, and for the PERP data set, we have $\varphi=\pi / 2$, therefore, the yield in equation (5.4) can be rewritten as a function of $\phi$ as

$$
\left(\frac{d N}{d \phi}\right)^{\|, \perp} \sim A(\phi) F^{\|, \perp}\left(1 \mp P^{\|, \perp} \Sigma \cos (2 \phi)\right)
$$

Using equation (5.5), the distribution $Y$ described in equation (5.3) can be rewritten as

$$
Y=\frac{1-F_{\mathrm{R}}+\frac{F_{\mathrm{R}} P_{\mathrm{R}}+1}{P_{\mathrm{R}}+1} 2 \bar{P} \sum \frac{\sin \Delta \phi}{\Delta \phi} \cos \left(2\left(\phi-\phi_{0}\right)\right)}{1+F_{\mathrm{R}}+\frac{1-F_{\mathrm{R}} P_{\mathrm{R}}}{P_{\mathrm{R}}+1} 2 \bar{P} \sum \frac{\sin \Delta \phi}{\Delta \phi} \cos \left(2\left(\phi-\phi_{0}\right)\right)},
$$

with the flux ratio $F_{\mathrm{R}}=\frac{F^{\|}}{F^{\perp}}$, polarization ratio $P_{\mathrm{R}}=\frac{P^{\|}}{P^{\perp}}$, and average of the polarization $\bar{P}=\frac{P^{\|}+P^{\perp}}{2}$. The $\phi_{0}$ parameter is introduced to account for the offset of the photon polarization vector. $\frac{\sin \Delta \phi}{\Delta \phi}$ is a correction for the bin of width $\Delta \phi$. 


\subsubsection{Parameter Optimization}

Several studies have been carried out testing the $\phi$-bin method ([44] [45]). Given the statistical restrictions, it is necessary to fix the values of the most of the parameters to have a more accurate estimate of the observable $\Sigma$. These parameters are:

- the offset of the photon polarization vector $\phi_{0}$,

- the flux ratio $F_{\mathrm{R}}$,

- the level of polarization ratio $P_{\mathrm{R}}$ and the average polarization $\bar{P}$.

\section{$\phi_{0}$ Offset}

This parameter gives information about the offset in the alignment of the diamond radiator in the goniometer. Since $\phi_{0}$ is a mechanical parameter, we do not expect it to change during the experiment except for diamond realignments. Also, $\phi_{0}$ does not depend on the channel and can be extracted accurately from a highstatistics channel from a fit on the asymmetry integrated over a large kinematic region. The channel with very high statistics was the single-pion channel $(\gamma n \rightarrow$ $p \pi^{-}$) worked by collaborator Daria Sokhan. The value reported is a weighted mean $0.03^{\circ} \pm 0.071^{\circ}$ ranging from maximum values of $\pm 2^{\circ}$.

\section{Photon Polarization}

The degree of photon polarization has to be determined to fix the two parameters

$P_{R}$ and $\bar{P}$. The photon polarization was obtained by fitting the enhancement distributions with a realistic theoretical model of the coherent spectrum [46]. The 
enhancement distribution is a common quantity used in these types of experiments, and it is calculated by dividing the coherent photon spectrum by the incoherent spectrum obtained with an amorphous radiator (in this case, the carbon radiator). This quantity, since it is a ratio, removes systematic effects and makes the determination of the photon polarization more reliable. The method used for this calculation takes into account the electron beam energy, the smear from beam collimation, beam size, fluctuations of the beam position and angle, and multiple scattering [27]. In figure 5.3, it is possible to see a typical enhancement distribution and a calculated degree of photon polarization. A program calculating the degree of polarization of the photon, on an event-by-event basis, was developed by the g13 collaborator Nicholas Zachariou. An overview of the steps for the method used to calculate the level of polarization is given below:

- The enhancement is calculated by dividing the coherent-photon-energy spectrum by the incoherent spectrum. Event-by-event data were used to account for the effect of collimation.

- The exact position of the coherent edge was calculated by fitting the enhancement in the region of the edge with a $3^{\text {rd }}$-order polynomial. The energy equivalent to the biggest slope in the enhancement was chosen as the energy of the coherent edge.

- Drift in the coherent peak position forced to bin the data as a function of the beam position. The bin width was $2 \mathrm{MeV}$ and the photon polarization was determined for each bin.

- The function used to fit the enhancement distribution is characterized by 5 parameters. These parameters account for the angle between the beam 
and the crystal plane defined by the [022] direction, the beam divergence, relative angle of collimation (in characteristic angle units), the smearing factor for collimation, and the amplitudes of the discrete peaks. An initial fitting for the coherent-edge-position bin with higher statistics was done to fix two of the five parameters in the fit.

- The three floating parameters are calculated for the other coherent-edgeposition bins.

- The level of photon polarization is calculated by an analytic function using the parameters extracted in the fitting routine.

- The level of polarization is then plotted as a function of the coherent edge position for each E-counter. In order to remove statistical fluctuations, the polarization is fitted with a $3^{\text {rd }}$-degree polynomial.

This method of determining the polarization is more accurate for E-counters located close to the nominal coherent edge.

This information was then stored in tables that give the average degree of linear polarization per run per E-counter. After the analysis cuts and event selection, the polarization average was calculated for each energy bin (a bin width of $200 \mathrm{MeV}$ under the coherent peak) and for each set: PARA for those with the electric field parallel to the lab floor, and PERP for those perpendicular to the floor. The values calculated are shown in table 5.1. The errors associated with the parameters reported in this section will be discussed in the next chapter. 

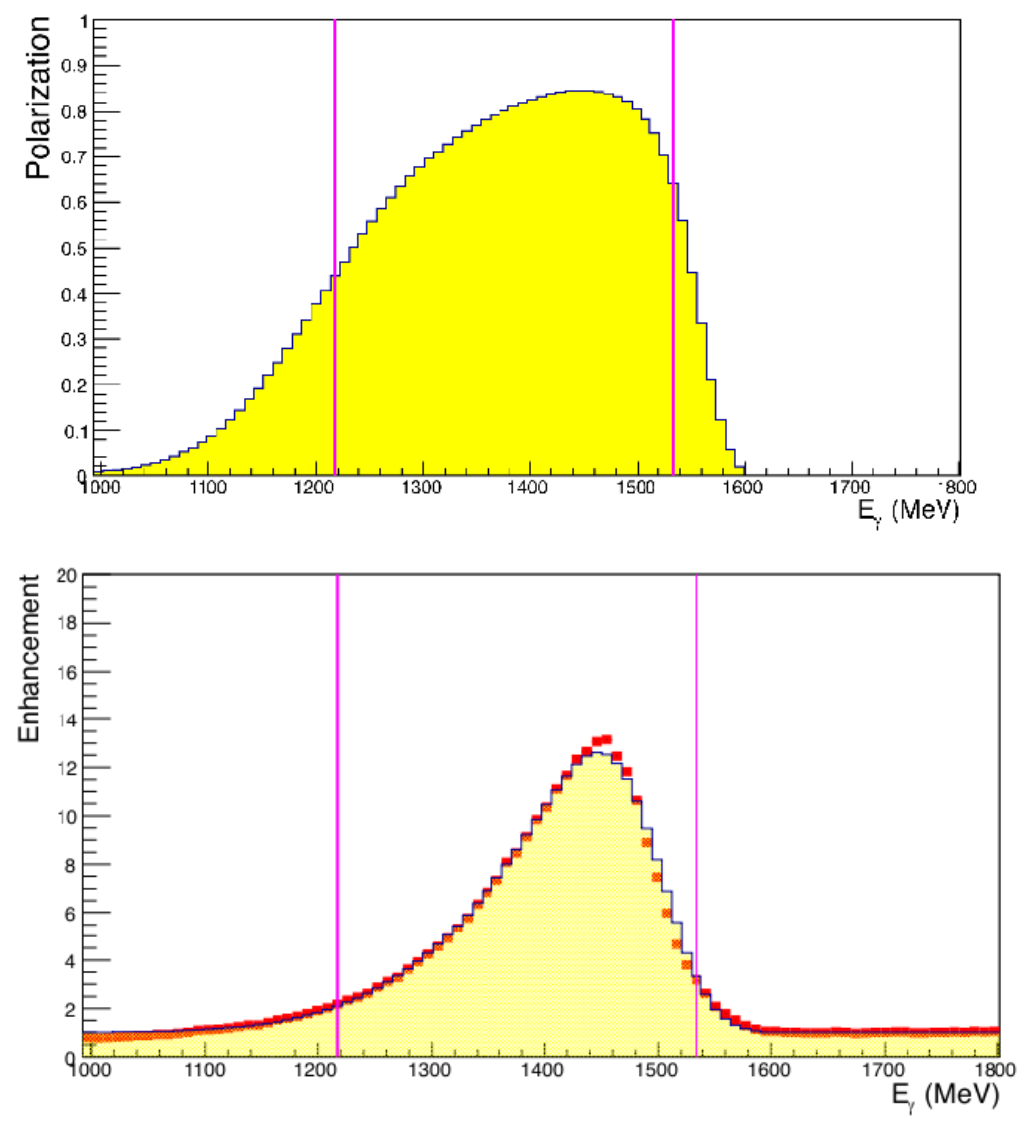

Fig. 5.3. Typical photon polarization (top) and corresponding enhancement distribution (bottom).

\begin{tabular}{|c|c|c|}
\hline$E_{\gamma}(\mathrm{GeV})$ & $P_{R}$ & $\bar{P}$ \\
\hline \hline $1.1-1.3$ & 0.88 & 0.754 \\
\hline $1.3-1.5$ & 1.01 & 0.782 \\
\hline $1.5-1.7$ & 0.96 & 0.750 \\
\hline $1.7-1.9$ & 0.94 & 0.676 \\
\hline $1.9-2.1$ & 0.99 & 0.730 \\
\hline $2.1-2.3$ & 1.02 & 0.695 \\
\hline
\end{tabular}

Table 5.1. Polarization ratio and polarization average for all energy subsets with different coherent peak. 


\section{Flux Ratio}

The flux ratio determination is based on a fit of the $\mathrm{Y}$ distribution, equation (5.3), with the function described in equation (5.6). Since the incident photon flux is constant within each energy subset, the integration over the entire polar-angle range and two bins in energy (200 $\mathrm{MeV}$ from the coherent edge nominal value) will provide good statistics for extraction of the parameter and for reducing any statistical fluctuations. An example of the fit performed is shown in figure 5.4 and the results are summarized in table 5.2. The statistics for this fit are larger than those that were used to calculate the beam asymmetry; the reduced $\chi^{2}$ was used to estimate the goodness of the fit. As can be seen in table 5.2, in general the values for the reduced $\chi^{2}$ are around 1 .

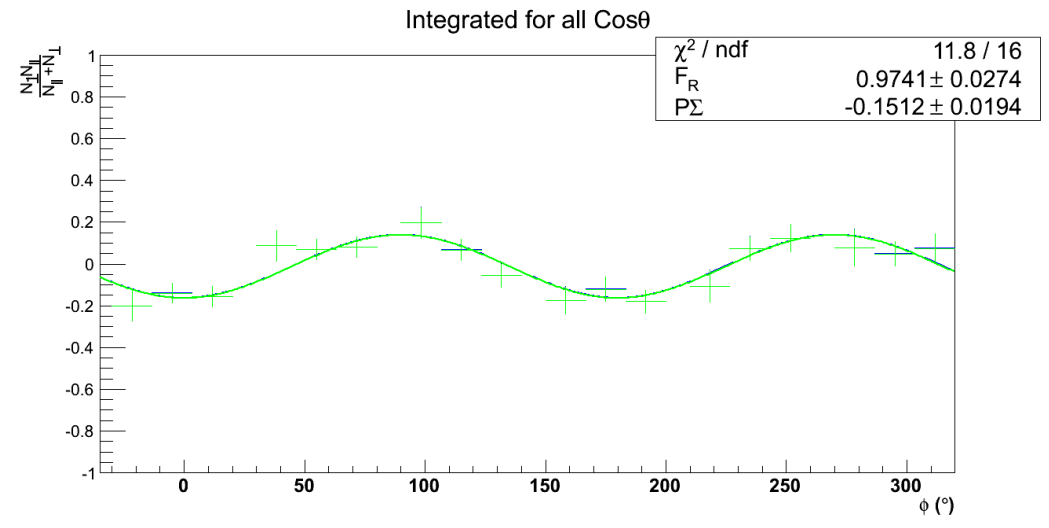

Fig. 5.4. Example of fit for $1.7<E_{\gamma}<1.8 \mathrm{GeV}$.

\subsubsection{Dilution Factor.}

The sample of the events, after all the cuts described in the previous chapter, still contains some background. Two possible kinds of background could be under the peak: polarized and unpolarized events. If the background is unpolarized, 


\begin{tabular}{|c|c|c|}
\hline$E_{\gamma}(\mathrm{GeV})$ & $F_{R}$ & $\chi^{2} / N D F$ \\
\hline \hline $1.1-1.3$ & $0.485 \pm 0.015$ & 1.098 \\
\hline $1.3-1.5$ & $1.024 \pm 0.015$ & 1.325 \\
\hline $1.5-1.7$ & $1.198 \pm 0.014$ & 1.358 \\
\hline $1.7-1.9$ & $0.914 \pm 0.009$ & 0.875 \\
\hline $1.9-2.1$ & $1.056 \pm 0.011$ & 0.677 \\
\hline $2.1-2.3$ & $1.058 \pm 0.012$ & 0.727 \\
\hline
\end{tabular}

Table 5.2. Calculated values for the flux ratio $F_{R}$.

a dilution factor can be defined to understand how much the background will "dilute" the results of extracting $\Sigma$. A background region was considered for values of invariant mass squared larger than an upper cut. This cut was defined before by the second-degree polynomial fitted over $\mu_{i}+3 \sigma_{i}$, where $\mu_{i}$ and $\sigma_{i}$ are the mean and the standard deviation of the Gaussian distribution describing the peak in the $i$ th bin of missing mass squared, respectively . After calculating $\Sigma$ in the background region, we found it to be 0 within the uncertainty. This is to say, the background is not polarized. To account for the remaining background under the peak after the cuts described above, a dilution factor D was defined for each energy subset,

$$
\begin{gathered}
D \equiv \frac{\sum_{i}\left(A_{\mathrm{tot}}-A_{\mathrm{bkg}}\right)_{i}}{\sum_{i}\left(A_{\mathrm{bkg}}\right)_{i}} \\
\left(\frac{d N}{d \phi}\right)_{\text {signal }}^{\|(\perp)}=D^{\|(\perp)}\left(\frac{d N}{d \phi}\right)_{\text {peak }}^{\|(\perp)},
\end{gathered}
$$

where the sum $\sum_{i}$ runs over all the missing mass squared bins, the region of the signal defined as $M^{2}\left(\pi^{+} \pi^{-} \pi^{0}\right) \in\left[\mu_{i}-3 \sigma_{i}, \mu_{i}+3 \sigma_{i}\right]$ and the background region defined as outside of the signal region. $A_{\text {peak }}$ can be calculated through integrating the model or by integrating the histogram. The difference of the two methods is shown in figure 5.5. It is important to note that although the difference in 
value of the dilution factor between the two methods is larger for the energy bins corresponding to lower energies, it has minimal impact on the calculation of the beam asymmetry as shown in figure 5.5. This is to say that the fits all agree with each other. For this work, we chose to use the dilution factor calculated by integrating over the histogram. The estimated values of the dilution factor are summarized in table 5.3.

Taking into account the dilution factor, it is possible to rewrite the ratio $Y$ described in equation (5.3) as the ratio of the corrected distribution

$$
Y^{*} \equiv \frac{D^{\perp}\left(\frac{d N}{d \phi}\right)^{\perp}-D^{\|}\left(\frac{d N}{d \phi}\right)^{\|}}{D^{\perp}\left(\frac{d N}{d \phi}\right)^{\perp}+D^{\|}\left(\frac{d N}{d \phi}\right)^{\|}} .
$$

The error associated with the dilution factor parameter is discussed in the next chapter (refer to section 6.5 ). Specifically in the regions of 1.1 to $1.7 \mathrm{GeV}$, the dilution factor is small, meaning that there is a large number of background events diluting the signal peak.

\begin{tabular}{|c||c|c||c|c|}
\hline$E_{\gamma}(\mathrm{GeV})$ & $D_{\text {HISTO }}^{\|}$ & $D_{\text {FIT }}^{\|}$ & $D_{\text {HISTO }}^{\perp}$ & $D_{\text {FIT }}^{\perp}$ \\
\hline \hline $1.1-1.3$ & 0.571 & 0.679 & 0.603 & 0.657 \\
\hline $1.3-1.5$ & 0.606 & 0.619 & 0.611 & 0.621 \\
\hline $1.5-1.7$ & 0.601 & 0.606 & 0.605 & 0.607 \\
\hline $1.7-1.9$ & 0.661 & 0.661 & 0.661 & 0.660 \\
\hline $1.9-2.1$ & 0.730 & 0.736 & 0.736 & 0.738 \\
\hline $2.1-2.3$ & 0.779 & 0.776 & 0.769 & 0.773 \\
\hline
\end{tabular}

Table 5.3. Dilution factor for each energy subset for PARA and PERP polarization. HISTO and FIT stand for integration over the histogram, and integration over the double-Gaussian model. 


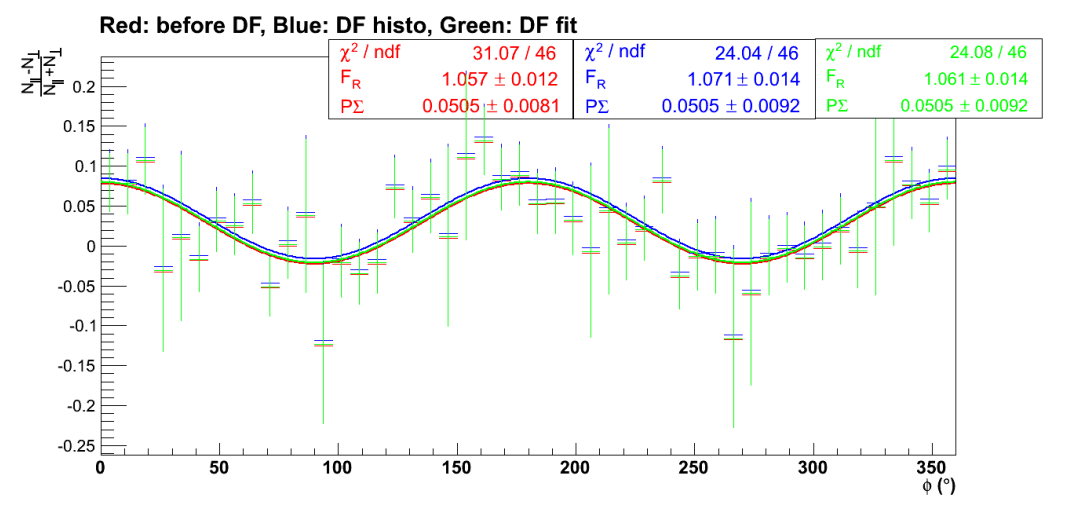

Fig. 5.5. Fit for the asymmetry extraction. The red dots and fit are taken before the dilution factor correction is applied. The blue dots and fit are calculated after the dilution factor is estimated by integrating the histogram. The green dots and fit are calculated after the dilution factor is estimated by integrating a double-Gaussian-fit model to the background.

\subsection{Statistical Uncertainty}

The extraction of the beam asymmetry $\Sigma$ uses a fit to the azimuthal yield ratios (refer to equation 5.3) as explained in section 5.2. This type of experiment has an intrinsic uncertainty because of the kinematical random nature of the nuclear processes ${ }^{3}$, which implies that the number of counts in each kinematic bin is a random variable. The Poisson distribution is typically associated with counting experiments; this means that the uncertainty associated with a specific $i$ th kinematic bin is $\sqrt{N_{i}}$. The statistical uncertainty of the ratio, modified by the dilution factor (refer to equation $(5.2 .2)$ ), is calculated by propagating the error of each bin, given by the expression,

$$
\sigma_{Y^{*}}=\frac{2 D^{\|} D^{\perp} \sqrt{\left.\left(\frac{d N}{d \phi}\right)^{\perp}\left(\frac{d N}{d \phi}\right)^{\|}\left(\frac{d N}{d \phi}\right)^{\perp}+\left(\frac{d N}{d \phi}\right)^{\|}\right)}}{D^{\perp}\left(\frac{d N}{d \phi}\right)^{\perp}+D^{\|}\left(\frac{d N}{d \phi}\right) \|} .
$$

\footnotetext{
${ }^{3}$ It is not possible to predict the kinematics value of the final-state particles.
} 
In equation (5.8), it is assumed that $\sigma_{D}^{\|, \perp}=0$. The uncertainty coming from the dilution factor calculation is included in the systematic uncertainties and will be discussed in the next chapter. Furthemore, we shall assume that $F^{\|}=F^{\perp}$ and $P^{\|}=P^{\perp}=\bar{P}$. Again, the uncertainties from the photon flux ratio $\sigma_{F_{R}}$ and the degree of polarization $\bar{P}$ are ignored and will be folded into the systematic uncertainties. Therefore, the statistical uncertainty in the asymmetry parameter is,

$$
\sigma_{\Sigma}=\frac{\sigma_{Y^{*}}}{\bar{P}}
$$

\subsection{Results}

After the optimization was performed for all the parameters, the $Y^{*}$ distribution defined in equation (5.2.2) was fit with the function described by equation (5.6). The product of the average polarization and the beam asymmetry parameter, $\bar{P} \Sigma$, is the only parameter that is left to float. The $\phi$ binning for the azimuthal distributions is a trade-off between describing the modulation correctly while having decent statistics. The regions where the coils are located have very low statistics and therefore have been excluded from the distribution. The bin width $\Delta \phi$ was studied for three different scenarios: two, three, and four bins per sector. The final binning choice was three $\phi$ bins per sector. In figure 5.6 it is possible to see the fit applied to $Y^{*}$ for the kinematic bin $E_{\gamma} \in[2.1,2.2] \mathrm{GeV}$.

Result $^{4}$ for Beam Spin Asymmetry for $1.1<E_{\gamma}<2.3 \mathrm{GeV}$ in energy bins of $\Delta E_{\gamma}=100 \mathrm{MeV}$ was calculated. The results are shown in figure 5.7. The blue triangles are the results of this work, and the red circles are those reported by GRAAL 2015 [17] also on the quasi-free photoproduction. The green squares are

\footnotetext{
${ }^{4}$ CLAS preliminary result.
} 

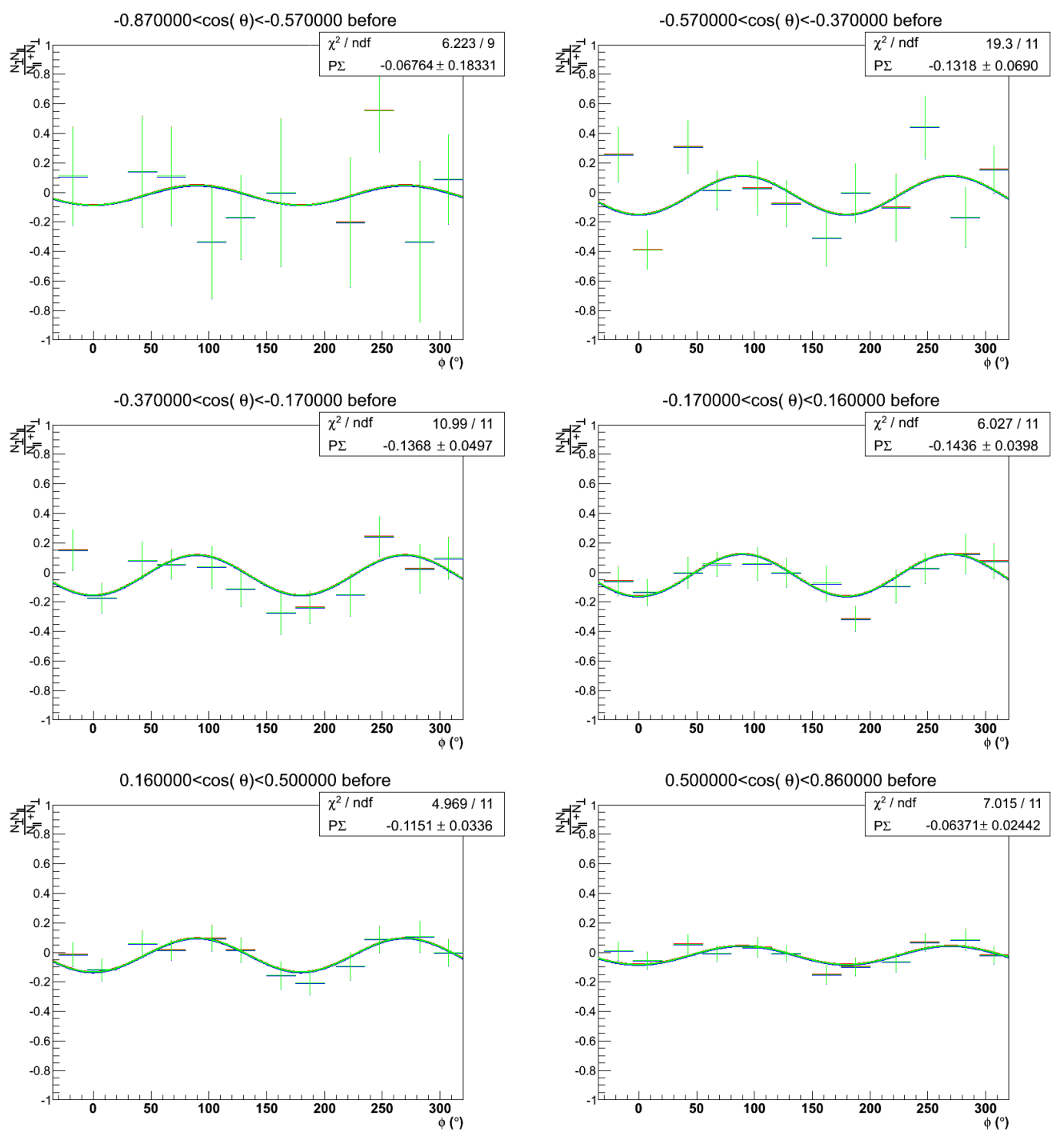

Fig. 5.6. Fitting the modulation of the ratio $Y^{*}$ for different kinematic bins in $\cos \theta_{\mathrm{CM}}$. 
the results of CLAS 2017 [14] reported for the photoproduction of the $\omega$ meson off the free proton. The error bars reflect only the statistical uncertainty and are calculated as indicated in equation (5.9).

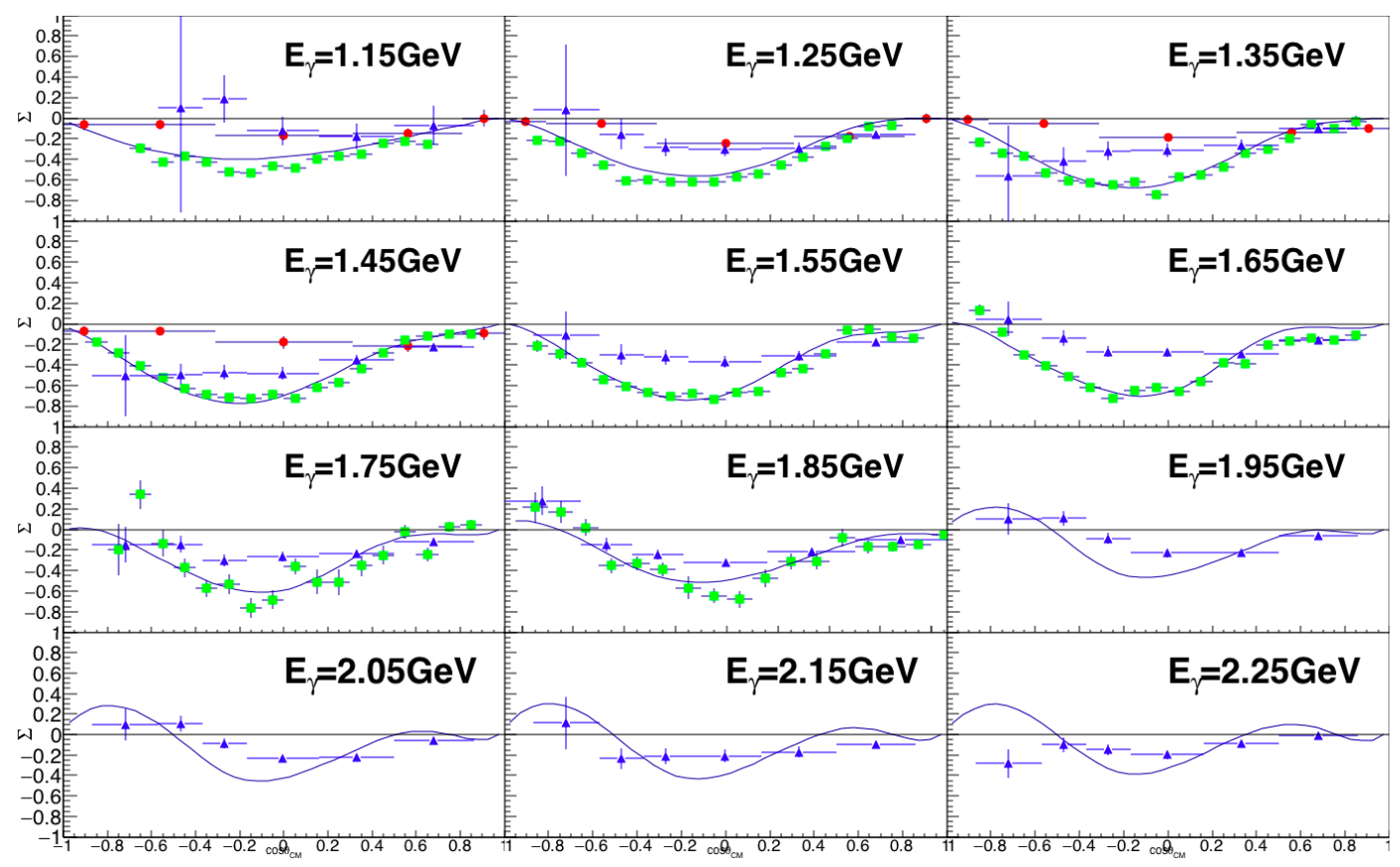

Fig. 5.7. Result for Beam Spin Asymmetry for $1.1<E_{\gamma}<2.3 \mathrm{GeV}$ in energy bins of $\Delta E_{\gamma}=200 \mathrm{MeV}$. The blue triangles represent this work (quasi-free proton), the red circles represent the GRAAL 2015 [17] results for the quasifree reaction. The green squares are the results from [14] for the free-proton channel. The blue line represents the Bonn-Gatchina prediction after including the $\omega$ photoproduction data from CLAS 2017 [14] in their fit. 


\section{Chapter 6}

\section{Systematic Studies}

\subsection{Overview}

After the best estimate of the observable $\Sigma$, it is of fundamental importance to quantify how good the estimate actually is. In general, there are two main types of uncertainties associated with the experimental estimation of a value: statistical and systematic. The statistical uncertainty has already been discussed in the previous chapter, where it took into account the random nature of the nuclear processes and the amount of events analyzed.

Systematic uncertainty, on the other hand, does not depend on the number of events, and it accounts for biases from nonstatistical sources. This chapter focuses on the quantification of the systematic uncertainties. We report each source of systematic uncertainty separately. 


\subsection{Goodness of the Fit}

In order to account for the goodness of the fit, we calculated the normalized residuals as seen in figure 6.1. The mean value for the distribution of the normalized residual is expected to be around zero, which can be seen in the plots. We added all the residuals for the six fits for each energy bin. In order to find the $\sigma^{2}$, we should fit a Gaussian to the normalized residual distribution for each energy and cosine bin. The limitation with this calculation is that we have only 12 points per distribution. The values for the $\sigma^{2}$ are estimated to be, in general, smaller than 1 . This is attributed to the fact that we have limited statistics.

For the two lowest values for energy bin and the backward angles, we found a bias. These two bins are the ones with the least statistics in all the data sets. This matter must be studied before publication of the results found in this thesis.
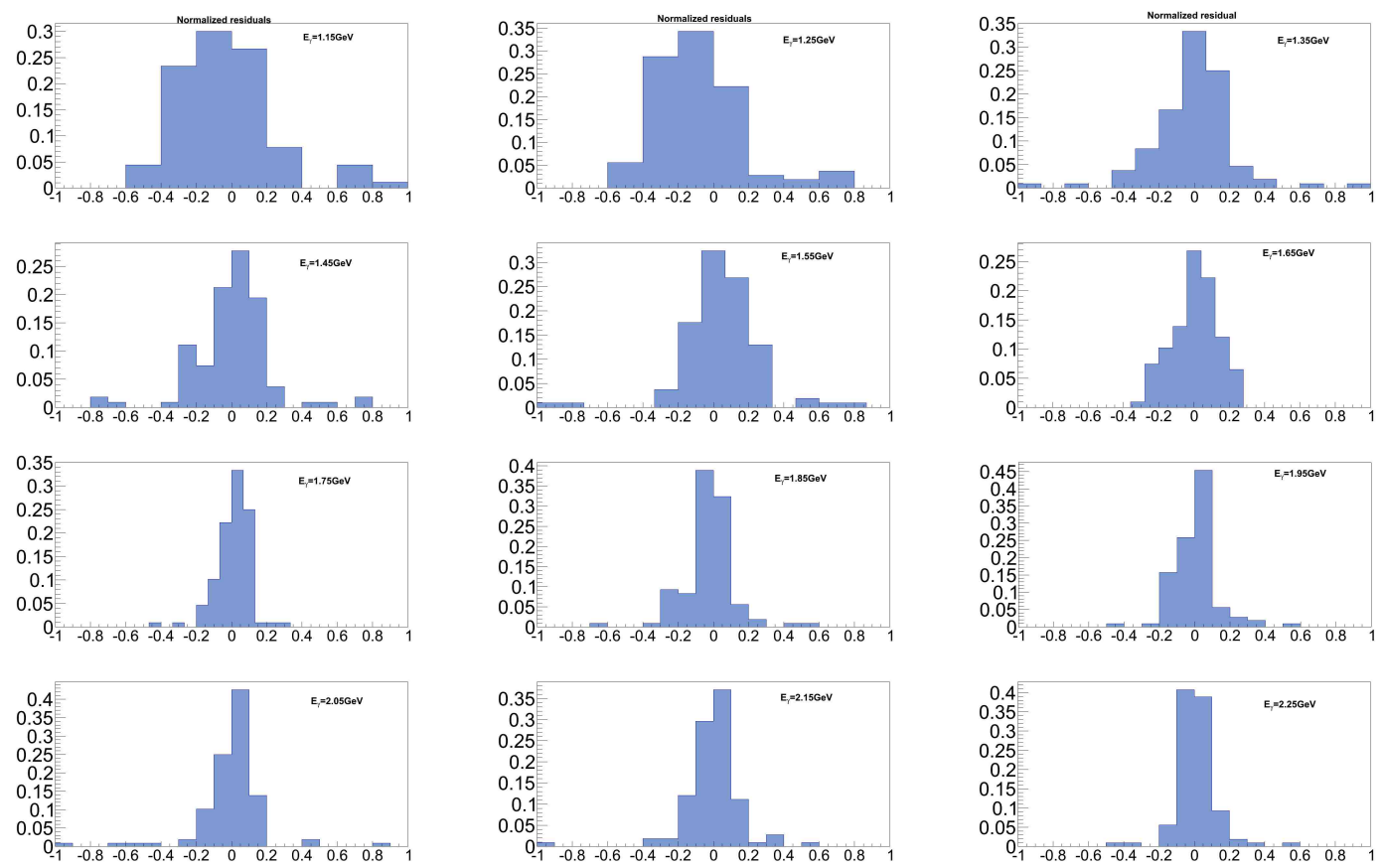

Fig. 6.1. Normalized residuals distribution for each energy bin. 


\subsection{Systematics of the Parameters}

To understand the robustness and to identify possible biases of the estimate of $\Sigma$, a detailed study of the sensitivity of the observable to the fit parameters was reported in the CLAS note [45]. In general, ideal probability distribution functions (pdfs) for PERP and PARA events were generated by sampling the azimuthal pdf. In other words, events outside the azimuthal pdf were discarded. These studies depend on the method and are not specific to a determined channel. In this section, this work is summarized.

\subsection{1 $\phi_{0}$ Offset}

The systematic uncertainty in the $\phi$-bin method $^{1}$ associated with the goniometer has been previously investigated for the $\phi$-bin method. As described in reference [45], studies using Monte Carlo-generated events with PARA and PERP polarization were used to calculate the uncertainty in the method. By varying several parameters such as $\Sigma, \phi_{0}$, and the number of events, the estimator of the total systematic uncertainty was chosen to be the standard deviation of the observable $\sigma_{\text {ТОт }}$. The "experiment" was repeated for a constant value of $\phi_{0}$ to account for statistical fluctuations, and the mean value was chosen to be the best estimate of statistical fluctuation $\sigma^{\mathrm{STAT}}$. The $\sigma_{\mathrm{sys}}=\sqrt{\sigma_{\mathrm{TOT}}^{2}-\sigma_{\mathrm{STAT}}^{2}}$ was reported to be of the order of $10^{-6}$. In this work, we shall use this value as our estimate. More studies on the $\Sigma$ dependency from the $\phi_{0}$ also carried out by our collaborator Nicholas Zachariou (see reference [45]); all coincide with this general estimate.

\footnotetext{
${ }^{1}$ The name of the method comes from reference [45]
} 


\subsubsection{Photon Flux}

A study, similar to the one described for $\phi_{0}$, was used to determined $F_{\mathrm{R}}$. The systematic uncertainty reported in reference $[47]$ is

$$
\sigma_{F_{\mathrm{R}}}^{\text {sys }}=0.073 \sigma_{F_{\mathrm{R}}}^{\mathrm{fit}}
$$

For this work, we report the systematic uncertainty for each data subset in table 6.1 , with values of the order of $\sigma_{F_{\mathrm{R}}}^{\mathrm{sys}} \sim 10^{-3} \cdot \sigma_{F_{\mathrm{R}}}^{\mathrm{fit}}$ used for this calculation are shown in table 5.2.

\begin{tabular}{|c|c|}
\hline$E_{\gamma}(\mathrm{GeV})$ & $\sigma_{F_{R}}$ \\
\hline \hline $1.1-1.3$ & 0.0011 \\
\hline $1.3-1.5$ & 0.0011 \\
\hline $1.5-1.7$ & 0.0010 \\
\hline $1.7-1.9$ & 0.0007 \\
\hline $1.9-2.1$ & 0.0008 \\
\hline $2.1-2.3$ & 0.009 \\
\hline
\end{tabular}

Table 6.1. Calculated values for the systematic uncertainty related to the flux ratio $F_{\mathrm{R}}$

\subsubsection{Photon Polarization Ratio}

The degree of photon polarization uncertainty affects two parameters, $P_{\mathrm{R}}$ and $\bar{P}$. The former is one of the parameters that is fixed in the fit, while the latter is a multiplicative parameter that affects directly the value of $\Sigma$. Since the extraction of both quantities comes from the same polarization tables, the calculation of the two parameters is correlated. In an independent study [44], the estimate of the uncertainty for the level of polarization is $\sigma_{P} \sim 7 \%$. Since $P$ is not the parameter that is used directly in the fit, we have to propagate this error to calculate the 
uncertainty of the polarization ratio $\sigma_{P_{R}}$ and the polarization average ${\sigma_{\bar{P}}}^{2}$. The propagation of error of this value provides an expression for $\sigma_{P_{R}}$,

$$
\begin{aligned}
\sigma_{P_{R}} & =P_{\mathrm{R}} \sqrt{\left(\frac{\Delta P^{\|}}{P^{\|}}\right)^{2}+\left(\frac{\Delta P^{\perp}}{P^{\perp}}\right)^{2}} \\
& =0.1 P_{\mathrm{R}} .
\end{aligned}
$$

A similar study [47] as the previous two found that the uncertainty of the beam asymmetry due to $P_{R}, \sigma_{P_{R}}^{\text {sys }}<1 \%$. On the other hand, the uncertainty of the average degree of polarization is given by

$$
\begin{aligned}
\sigma_{\bar{P}} & =\frac{1}{2} \sqrt{\left(\Delta P^{\|}\right)^{2}+\left(\Delta P^{\perp}\right)^{2}} \\
& \sim 0.05 \bar{P} .
\end{aligned}
$$

The uncertainty reported in that work is $\sigma_{\bar{P}}^{\text {sys }}=5 \%$, and we shall use this value as our estimate.

\subsection{Event Selection Systematic Uncertainty}

Unlike the previous uncertainties reported above, the ones that are directly related to the event selection cuts are channel dependent. For the estimate of these values, we did not make use of an event generator. Instead, the cuts were varied from their nominal value to broader or tighter cuts. The beam asymmetry was then calculated again leaving all the other parameters fixed. The total difference $\Delta \Sigma \equiv \Sigma^{*}-\Sigma$ between the value of the beam asymmetry calculated with different cuts $\left(\Sigma^{*}\right)$ and the beam asymmetry calculated with the nominal cuts $\Sigma$, was then

\footnotetext{
${ }^{2}$ It is important to note that $\sigma_{P_{R}}$, and $\sigma_{\bar{P}}$ are the uncertainties that will be reported in this work. The uncertainty of the level of polarization $\sigma_{P}$ is an intermediate step
} 
fitted with a 0 -degree polynomial. The value reported by the difference was then selected as the maximum systematic uncertainty for that cut.

\subsubsection{Neutral Particle Identification Systematics}

The cut for neutral particle identification as a photon was done by asking the relative velocity of both neutral tracks to be $\beta>0.95$. For this study, we performed a looser cut asking $\beta>0.9$. The systematic uncertainty related to this cut is reported as 0.017 , as can be seen in figure 6.2 .

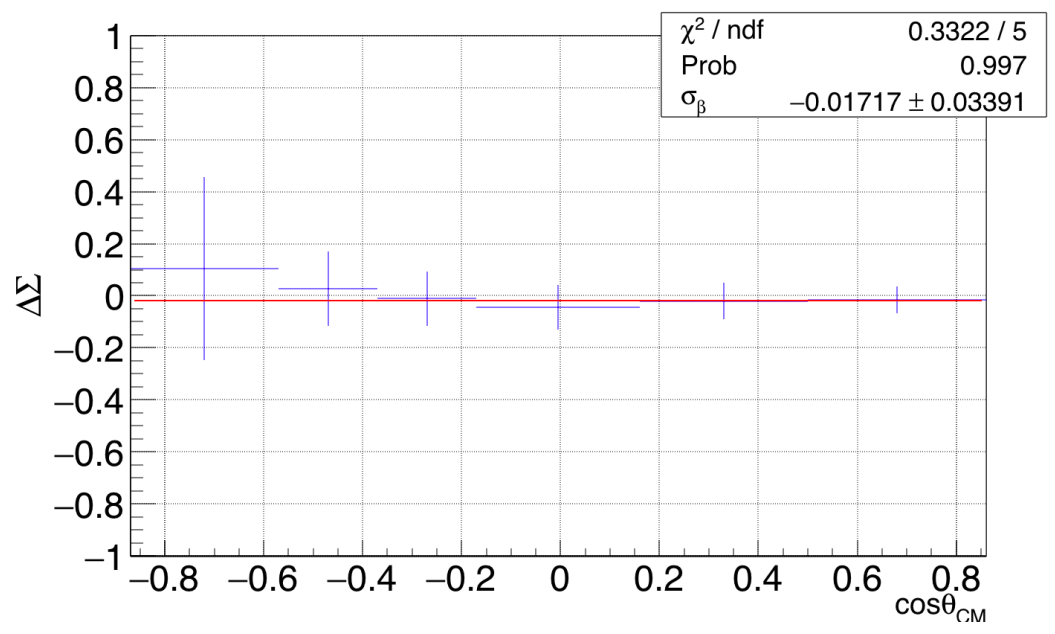

Fig. 6.2. $\Delta \Sigma$ as a function of $\cos \theta_{C M} . \Sigma^{*}$ is calculated using a broader cut on the neutral particle identification $\left(\beta_{n}<0.9\right)$.

\subsubsection{Incident Photon Identification}

For this study, the cut variation was set to be $\Delta t<1.2$ ns as opposed to the $1 \mathrm{~ns}$ nominal cut. The difference is shown in figure 6.3 and the value reported is $\sim 0.001$ 


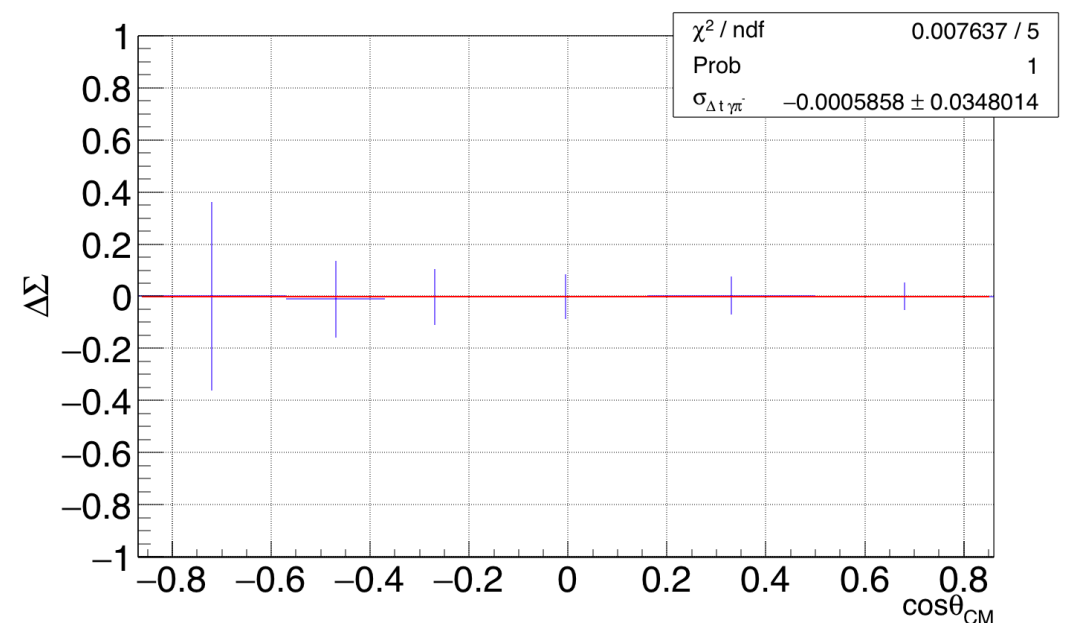

Fig. 6.3. $\Delta \Sigma$ as a function of $\cos \theta_{C M} . \Sigma^{*}$ is calculated using a broader cut on the incoming photon coincidence time with $\pi^{-}\left(\Delta t_{\gamma \pi^{-}} \leq 1.2 \mathrm{~ns}\right)$.

\subsubsection{Out-of-Time Cut}

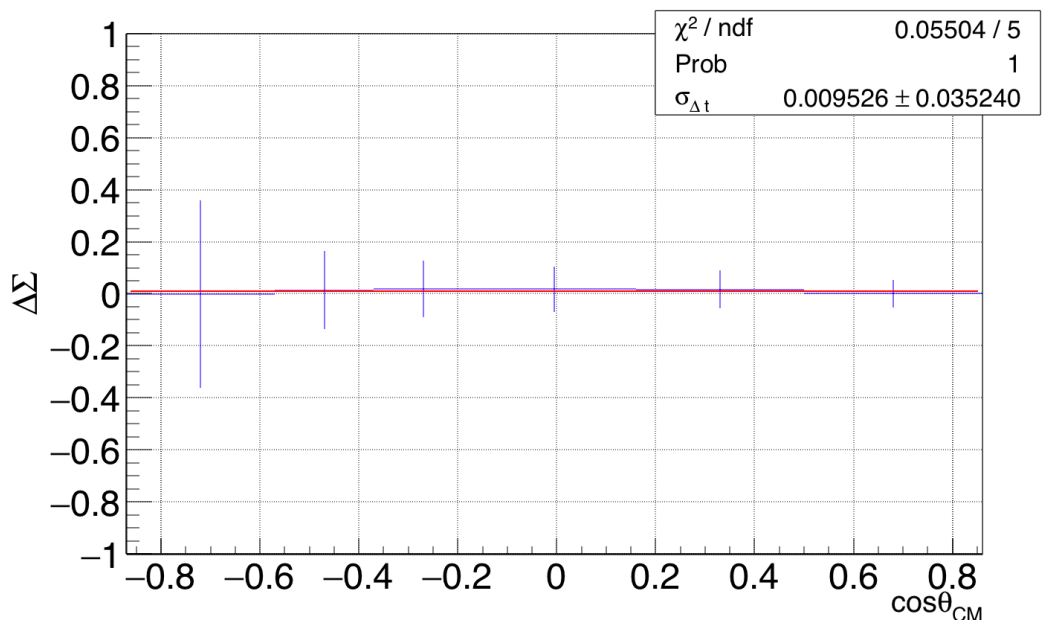

Fig. 6.4. $\Delta \Sigma$ as a function of $\cos \theta_{C M} . \Sigma^{*}$ is calculated using a tighter cut on the proton and $\pi^{+}$coincidence time with $\pi^{-}\left(\Delta t_{h \pi^{-}} \leq 1.0 \mathrm{~ns}\right)$.

To estimate the systematic uncertainty associated with the out-of-time hadrons cut, a variation was set to be $\Delta t<1.0 \mathrm{~ns}$ as opposed to the 1.2-ns nominal cut. 
The change of the cut was applied simultaneously to $\Delta t\left(\pi^{-} p\right)$ and $\Delta t\left(\pi^{-} \pi^{+}\right)$. The difference is shown in figure 6.4 and the value reported is $\sigma_{\Delta t h h}=0.009$.

\subsection{4 z-Vertex Cut}

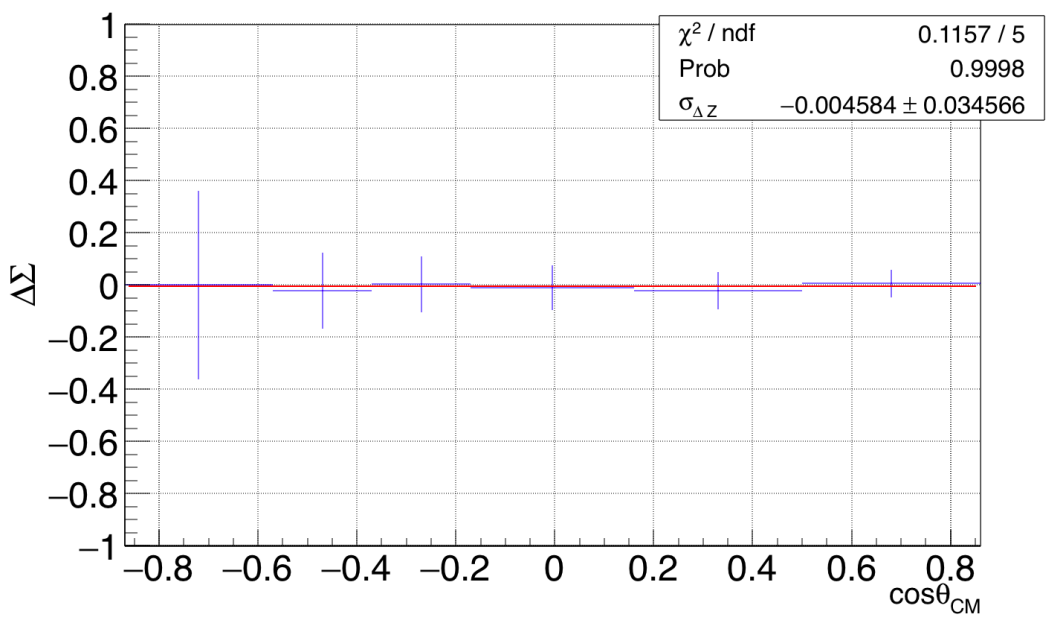

Fig. 6.5. $\Delta \Sigma$ as a function of $\cos \theta_{C M} . \Sigma^{*}$ is calculated using a broader cut on the $z$ coordinate of the primary vertex $(-40 \leq z \leq 0 \mathrm{~cm})$.

A broader cut for the coordinate $z$ of the primary vertex was done. The nominal values were instead taken into account, $-40 \leq z \leq 0 \mathrm{~cm}$. The $\Delta \Sigma$ is shown in figure 6.5, and the absolute difference associated with this cut is $\sigma_{z}=0.005$

\subsubsection{Missing Momentum}

The missing momentum cut is fundamental for identifying quasi-free events from FSI dominated events. For this, a tighter cut in missing momentum $P_{X}<$ $0.15 \mathrm{GeV} / \mathrm{c}$ was chosen. The systematic reported here is 0.021 as seen in figure 6.6. 


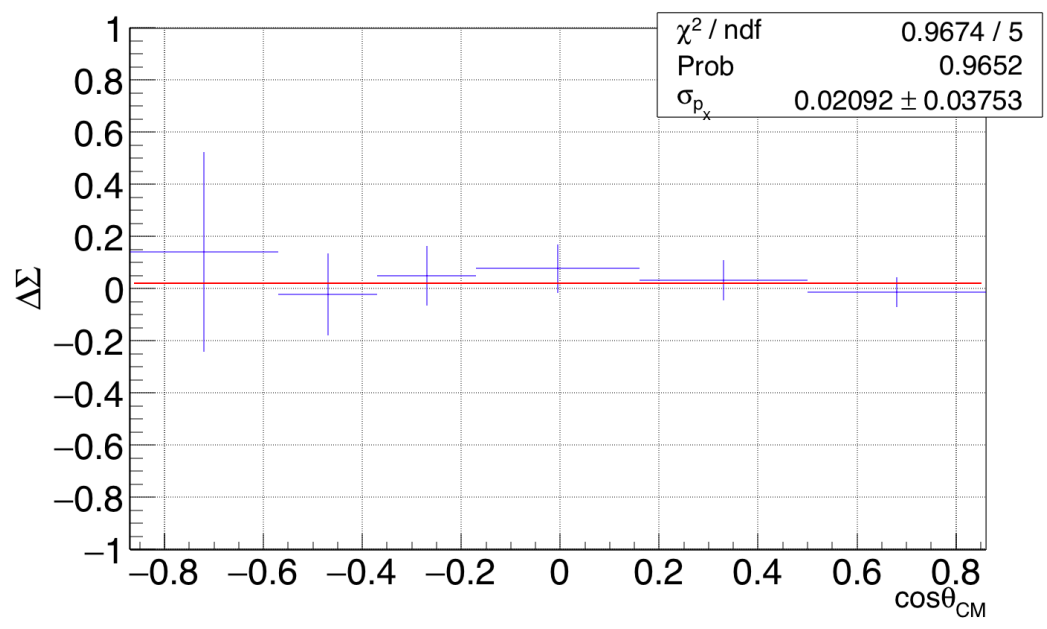

Fig. 6.6. $\Delta \Sigma$ as a function of $\cos \theta_{C M} . \Sigma^{*}$ is calculated using a tighter cut on the missing momentum $\left(P_{X}<0.15 \mathrm{GeV} / \mathrm{c}\right)$.

\subsection{Systematic due to Dilution Factor and In- variant Mass Cut}

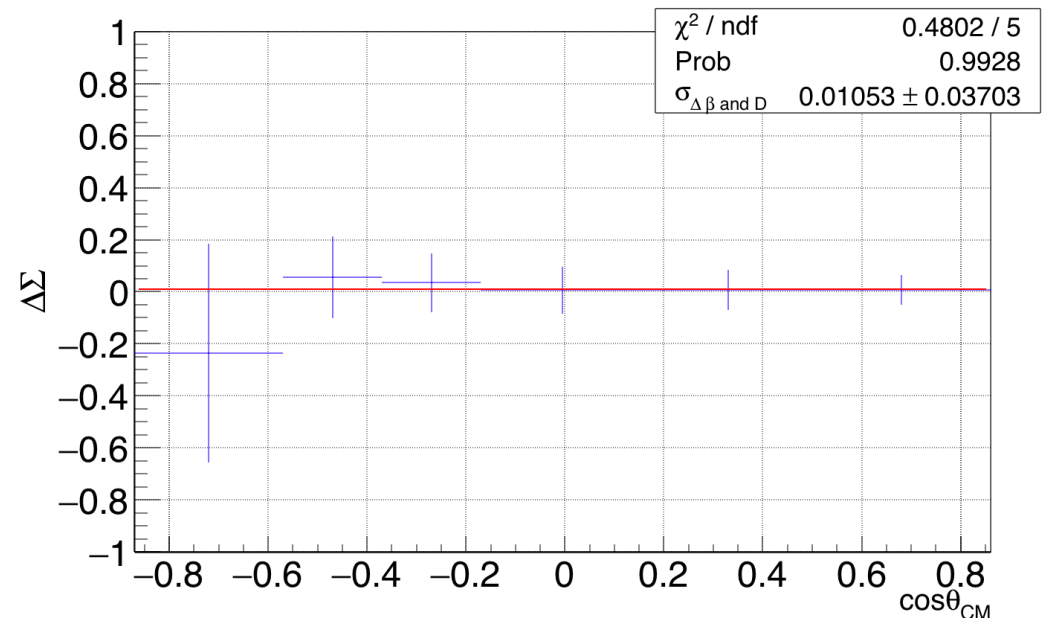

Fig. 6.7. $\Delta \Sigma$ as a function of $\cos \theta_{C M} . \Sigma^{*}$ is calculated using a tighter $2-\sigma$ cut on the calculation of the dilution factor.

The study of the systematic uncertainty related to the calculation of the dilu- 
tion factor is twofold; on the one hand, we have to estimate the error associated with the way we calculated the parameter, and on the other hand, we have to propagate this uncertainty into the $\sigma_{\Sigma}$. We performed a separation from the background with a $2 \sigma$ cut instead of the $3 \sigma$ cut to separate the systematic uncertainty from the statistical. This value accounts for the cut and the dilution factor calculation. For example, for the energy bin corresponding to $2.15 \pm 0.05 \mathrm{GeV}$ , the dilution factors for PARA and PERP with the $2 \sigma$ cut are $D^{\|}=0.832$ and $D^{\perp}=0.825$. These numbers are larger than the nominal values reported before as expected ${ }^{3}$. The final systematic estimate is 0.010 as shown in figure 6.7 .

\subsection{Summary and Conclusion}

The systematic uncertainty was calculated for different parameters, selection cuts, and background subtraction. The parameter that most influences the uncertainty is the determination of the degree of polarization with a value of $5 \%$ as explained in section 6.3.3. The uncertainties for each cut are minimal and are assumed to be independent. However, this assumption is not correct for the calculation of the dilution factor and the invariant-mass-squared cut. The uncertainty of these two aspects is highly correlated and is calculated as one unique source of error.

\subsubsection{Systematic Error Budget}

Table 6.2 is a summary of our estimate for the systematic uncertainty found in this work.

\footnotetext{
${ }^{3}$ As we us a tighter cut, the amount of events diluting the signal region should be smaller, increasing the value of the dilution factor.
} 


\begin{tabular}{|c|c|}
\hline Source of uncertainty & $\left|\mu_{\Delta \Sigma}\right|$ \\
\hline \hline$\phi_{0}$ offset & $10^{-6}$ \\
\hline Photon flux ratio & $\sim 0.001$ \\
\hline Polarization ratio & $<1 \%$ \\
\hline Mean polarization & $5 \%$ \\
\hline Neutral particle cut & 0.017 \\
\hline Incident photon identification & 0.001 \\
\hline Out of time cut & 0.009 \\
\hline$z$-vertex cut & 0.005 \\
\hline Missing momentum cut & 0.021 \\
\hline Dilution factor and $3-\sigma$ cut & 0.010 \\
\hline
\end{tabular}

Table 6.2. Summary of the estimated values for the systematic uncertainties related to different sources. As extensively explained in this chapter, the estimated systematic uncertainties related with the kinematical cuts are averages of the absolute difference of the parameter $\Sigma$ and its variation. 


\section{Chapter 7}

\section{Summary and Conclusions}

In this work, we extracted the polarization observable $\Sigma$ for the exclusive quasifree photoproduction of the $\omega$ meson off the bounded proton in deuterium. The photon energy range spans from 1.1 to $2.3 \mathrm{GeV}$, for energy bins of $100 \mathrm{MeV}$. Systematic studies to estimate the uncertainty are also included in Chapter 6 . The first visible remark is that the beam asymmetry is not 0 and it is negative for most of the kinematic bins, which was expected, as was explained in Chapter 2. This fact is in agreement with $s$-channel contributions. The comparison of my results with GRAAL (2015) [17] is shown in figure 5.7. Previous data from GRAAL also for the quasi-free photoproduction are also plotted in figure 5.7, represented as red circles. The blue triangles are the results of this thesis. There is, in general, good agreement for the two first energy bins. However, for $E_{\gamma}=$ $1.35 \mathrm{GeV}$ and $E_{\gamma}=1.45 \mathrm{GeV}$, the GRAAL results are systematically smaller than those presented here. On the other hand, our data are compared with the photoproduction of the $\omega$ meson off the free $\operatorname{proton}^{1}$ reported by CLAS [14]. The values for this unbound channel are systematically larger in amplitude than

\footnotetext{
${ }^{1}$ The hydrogen target, where the proton is not bound to any other nucleons in the nucleus.
} 
the ones presented in this work. This suggests that nuclear effects like the offshellness of the proton, Fermi motion, or the nucleon binding could possibly affect the polarization observable $\Sigma$. On the other hand, this could possibly come from final-state-interaction corrections that have to be taken into account even after the missing mass cut. CLAS 2017 [14] and GRAAL 2015 [17] are also in disagreement with respect to the free proton (the free proton target from CLAS is not shown here). There are no published data so far for the energy bins from $1.95 \mathrm{GeV}$ to $2.25 \mathrm{GeV}$ for any experiment.

The blue line in figure 5.7 is the multi-channel coupled fit from the BonnGatchina collaboration. The results include many channels and observables. In particular, the Bonn-Gatchina fit includes the free proton data from CLAS 2017 and predicts the values for the last three bins. The data for large energies will be fitted to theoretical calculation and will offer a constraint on the Partial Wave Analysis performed by various groups. This information, in time, will be a valuable input for the search of higher mass missing resonances.

This study will also help with a greater understanding of the quasi-free events, which is of vital importance for the study of reactions with a bound neutron target. In this case, it is not possible to compare with the free neutron, and although in general we do not expect the quasi-free neutron to have exactly the same effect in the $\Sigma$, the methodology will be similar. 


\section{Bibliography}

1. S. Capstick, W. Roberts, Prog. Part. Nucl. Phys. 45, S241-S331 (2000).

2. J. J. Dudek, R. G. Edwards, P. Guo, C. E. Thomas, Phys. Rev. D88, 094505 (2013).

3. A. V. Anisovich et al., Mesons and Baryons (WORLD SCIENTIFIC, 2008), DOI: 10.1142/6871, eprint: https : //www . worldscientific . com/doi / pdf/10.1142/6871, https://www . worldscientific.com/doi/abs/10. $1142 / 6871$.

4. E. Klempt, J.-M. Richard, Rev. Mod. Phys. 82, 1095-1153 (2010).

5. M. Tanabashi et al., Phys. Rev. D 98, 030001 (2018).

6. M. Pichowsky, C. Savkli, F. Tabakin, Phys. Rev. C53, 593-610 (1996).

7. K. Schilling, P. Seyboth, G. E. Wolf, Nucl. Phys. B15, [Erratum: Nucl. Phys. B18, 332 (1970)], 397-412 (1970).

8. J. Ballam et al., Phys. Rev. D7, 3150 (1973).

9. M. Battaglieri et al., Phys. Rev. Lett. 90, 022002 (2003).

10. J. Barth et al., Eur. Phys. J. A18, 117-127 (2003).

11. M. Williams, Nucl. Phys. Proc. Suppl. 186, 129-132 (2009).

12. M. Williams et al., Phys. Rev. C80, 065208 (2009). 
13. A. Wilson et al., Phys. Lett. B749, 407-413 (2015).

14. P. Collins et al., Phys. Lett. B773, 112-120 (2017).

15. F. Klein et al., Phys. Rev. D78, 117101 (2008).

16. J. Ajaka et al., Phys. Rev. Lett. 96, 132003 (2006).

17. V. Vegna et al., Phys. Rev. C91, 065207 (2015).

18. Q. Zhao, Z.-p. Li, C. Bennhold, Phys. Rev. C58, 2393-2413 (1998).

19. Q. Zhao, Nucl. Phys. A675, 217C-221C (2000).

20. Q. Zhao, Phys. Rev. C63, 025203 (2001).

21. C. W. Leemann, D. R. Douglas, G. A. Krafft, Ann. Rev. Nucl. Part. Sci. 51, 413-450 (2001).

22. B. A. Mecking et al., Nucl. Instrum. Meth. A503, 513-553 (2003).

23. U. Timm, Fortsch. Phys. 17, 765-808 (1969).

24. A. W. Sáenz, H. Überall, in Coherent Radiation Sources, ed. by A. W. Sáenz, H. Überall (Springer Berlin Heidelberg, Berlin, Heidelberg, 1985), pp. 5-6, ISBN: 978-3-642-51185-1, DOI: $10.1007 / 978-3-642-51185-1 \_2$, https://doi.org/10.1007/978-3-642-51185-1_2.

25. K. Livingston, Nucl. Instrum. Meth. A603, 205 (2009).

26. J. A. Salamanca Bernal, PhD thesis, Idaho State U., 2009, http://www . jlab.org/Hall-B/general/thesis/salamanca_thesis.pdf.

27. K. Livingston, CLAS-Note 020, http://nuclear.gla.ac.uk/ kl/mainz/ cbrem/FittingCoherentBrem.pdf (2011).

28. D. I. Sober et al., Nucl. Instrum. Meth. A440, 263-284 (2000).

29. Y. G. Sharabian et al., Nucl. Instrum. Meth. A556, 246-258 (2006). 
30. M. D. Mestayer et al., Nucl. Instrum. Meth. A449, 81-111 (2000).

31. E. S. Smith et al., Nucl. Instrum. Meth. A432, 265-298 (1999).

32. M. Amarian et al., Nucl. Instrum. Meth. A460, 239-265 (2001).

33. P. Nadel-Turonski, B. L. Berman, Y. Ilieva, D. G. Ireland, A. Tkabladze, Few Body Syst. 43, 227-232 (2008).

34. S. Christo, The Jefferson Lab Hall B Cryotarget Page, [Online; accessed 31January-2018], https : //userweb . jlab . org/ christo/g11a\%20target . html.

35. D. Lawrence, CLAS online BOS Bank Viewer, http://clasweb.jlab. org/bos/browsebos . php?build=centos $7 \% 2$ Ftrunk.

36. V. Blobel (1988).

37. J. Gilfoyle, Using the online version of RECSIS, https://userweb.jlab. org/ gilfoyle/recsis_online/recsis_online.html.

38. A. Tkvladze, Monitoring plots for g13, http : / / clasweb.jlab . org / rungroups/g13/monitor/monitorwrapper.html.

39. R. Brun, F. Rademakers, presented at the AIHENP'96 Workshop, Lausane, vol. 389, pp. 81-86.

40. W. Leo, Techniques for Nuclear and Particle Physics Experiments: A HowTo Approach (U.S. Government Printing Office, 1994), ISBN: 9783540572800 , https://books .google.com/books?id=8VufE4SD-AkC.

41. D. Keller, CLAS-Note 015 (2010).

42. P. Mattione et al., CLAS-Note 001 (2017).

43. M. Williams et al., CLAS-Note 17 (2004). 
44. K. Livingston, CLAS-Note 010 (2012).

45. N. Zachariou et al., CLAS-Note 011 (2012).

46. H. Überall, Phys. Rev. 103, 1055-1067 (4 1956).

47. N. Zachariou et al., Phys. Rev. C91, 055202 (2015). 